\title{
CONNECTING MANY-SORTED THEORIES
}

\author{
FRANZ BAADER AND SILVIO GHILARDI
}

\begin{abstract}
Basically, the connection of two many-sorted theories is obtained by taking their disjoint union, and then connecting the two parts through connection functions that must behave like homomorphisms on the shared signature. We determine conditions under which decidability of the validity of universal formulae in the component theories transfers to their connection. In addition, we consider variants of the basic connection scheme. Our results can be seen as a generalization of the so-called $\mathcal{E}$-connection approach for combining modal logics to an algebraic setting.
\end{abstract}

$\S 1$. Introduction. The combination of decision procedures for logical theories arises in many areas of logic in computer science, such as constraint solving, automated deduction, term rewriting, modal logics, and description logics. In general, one has two first-order theories $T_{1}$ and $T_{2}$ over signatures $\Sigma_{1}$ and $\Sigma_{2}$, for which validity of a certain type of formulae (e.g., universal, existential positive, etc.) is decidable. These theories are then combined into a new theory $T$ over a combination $\Sigma$ of the signatures $\Sigma_{1}$ and $\Sigma_{2}$. The question is whether decidability transfers from $T_{1}, T_{2}$ to their combination $T$.

One way of combining the theories $T_{1}, T_{2}$ is to build their union $T_{1} \cup T_{2}$. Both the Nelson-Oppen combination procedure [23, 22] and combination procedures for the word problem $[26,28,24,7]$ address this type of combination, but for different types of formulae to be decided. Whereas the original combination procedures were restricted to the case of theories over disjoint signatures, there are now also solutions for the non-disjoint case $[12,31,8,13,16,4,5]$, but they always require some additional restrictions since it is easy to see that in the unrestricted case decidability does not transfer. Similar combination problems have also been investigated in modal logic, where one asks whether decidability of (relativized) validity transfers from two modal logics to their fusion [19, 29, 32, 6]. The approaches in $[16,4,5]$ actually generalize these results from equational theories induced by modal logics to more general first-order theories satisfying certain model-theoretic restrictions: the theories $T_{1}, T_{2}$ must be compatible with their shared theory $T_{0}$, and this shared theory must be locally finite (a condition ensuring that finitely generated models are finite). The theory $T_{i}$ is compatible with the shared theory $T_{0}$ iff (i) $T_{0} \subseteq T_{i}$; (ii) $T_{0}$ has a model completion $T_{0}^{*}$; and (iii) every model of $T_{i}$ embeds into a model of $T_{i} \cup T_{0}^{*}$. 
In [20], a new combination scheme for modal logics, called $\mathcal{E}$-connection, was introduced, for which decidability transfer is much simpler to show than in the case of the fusion. Intuitively, the difference between fusion and $\mathcal{E}$-connection can be explained as follows. A model of the fusion is obtained from two models of the component logics by identifying their domains. In contrast, a model of the $\mathcal{E}$-connection consists of two separate models of the component logics together with certain connecting relations between their domains. There are also differences in the syntax of the combined logic. In the case of the fusion, the Boolean operators are shared, and all operators can be applied to each other without restrictions. In the case of the $\mathcal{E}$-connection, there are two copies of the Boolean operators, and operators of the different logics cannot be mixed; the only connection between the two logics are new (diamond) modal operators that are induced by the connecting relations.

If we want to adapt this approach to the more general setting of combining first-order theories, then we must consider many-sorted theories since only the sorts allow us to keep the domains separate and to restrict the way function symbols can be applied to each other. Let $T_{1}, T_{2}$ be two many-sorted theories that may share some sorts as well as function and relation symbols. We first build the disjoint union $T_{1} \uplus T_{2}$ of these two theories (by using disjoint copies of the shared parts), and then connect them by introducing connection functions between the shared sorts. These connection functions must behave like homomorphisms for the shared function and predicate symbols, i.e., the axioms stating this are added to $T_{1} \uplus T_{2}$. This corresponds to the fact that the new diamond operators in the $\mathcal{E}$-connection approach distribute over disjunction and do not change the false formula $\perp$. We call the combined theory obtained this way the connection of $T_{1}$ and $T_{2}$.

This kind of connection between theories has also been considered in automated deduction (see, e.g., $[1,33]$ ), but only in very restricted cases where both $T_{1}$ and $T_{2}$ are fixed theories (e.g., the theory of sets and the theory of integers in [33]) and the connection functions have a fixed meaning (like yielding the length of a list). In categorical logic, this type of connection can be seen as an instance of a more general co-comma construction, see for instance [34]. However, in this general setting, computational properties of the combined theories have not been considered yet.

This paper is a first step towards providing general results on the transfer of decidability from component theories to their connection. We start by considering the simplest case where there is just one connection function, and show that decidability transfers whenever certain model-theoretic conditions are satisfied. These conditions are weaker than the ones required in $[4,5]$ for the case of the union of theories. In general, these conditions are not weaker than the ones in [16], although all the theories satisfying the conditions in [16] that we have considered until now also satisfy our new conditions. Another advantage of the connection approach over the fusion approach for combining theories is that both the combination procedure and its proof of correctness are much simpler than the ones in $[16,4,5]$. 
The approach easily extends to the case of several connection functions. We will also consider variants of the general combination scheme where the connection function must satisfy additional properties (like being surjective, an embedding, an isomorphism), or where a theory is connected with itself. The first variant is, for example, interesting since the combination result for the union of theories shown in [16] can be obtained from the variant where one has an isomorphism as connection function. The second case is interesting since it can be used to reduce the global consequence problem in the modal logic $\mathbf{K}$ to propositional satisfiability, which is a surprising result.

This article is structured as follows. The next section introduces the notation and gives some important definitions, in particular the formal definition of the connection of two many-sorted theories. In addition, this section contains an example that demonstrates that decidability does not transfer in general from the component theories to their connection. $\S 3$ then introduces restrictions that ensure such a decidability transfer. $\S 4$ shows the main decidability transfer result for the case of one connection function and its extension to the case of several connection functions, possibly going in both directions. This section also provides examples of several (classes of) theories to which these results apply. $\$ 5$ treats the variant of the connection scheme where a theory is connected with itself, and $\S 6$ the variants that impose additional restrictions on the connection functions. $\S 7$ compares our algebraic approach for connecting many-sorted theories with the notion of an $\mathcal{E}$-connection, as introduced in [20]. $\S 8$ investigates the algebraic restrictions required for our decidability transfer results in more detail, and $\S 9$ uses the results of this section to provide alternative proofs for these transfer results.

§2. Notation and definitions. In this section, we fix the notation and give some important definitions, in particular a formal definition of the connection of two theories.

2.1. Many-sorted first-order logic. We use standard many-sorted firstorder logic (see, e.g., [14]), but try to avoid the notational overhead caused by the presence of sorts as much as possible. Thus, a signature $\Omega$ consists of a non-empty set of sorts $\mathcal{S}$ together with a set of function symbols $\mathcal{F}$ and a set of predicate symbols $\mathcal{P}$. The function and predicate symbols are equipped with arities from $\mathcal{S}^{*}$ in the usual way. For example, if the arity of $f \in \mathcal{F}$ is $S_{1} S_{2} S_{3}$, then this means that the function $f$ takes tuples consisting of an element of sort $S_{1}$ and an element of sort $S_{2}$ as input, and produces an element of sort $S_{3}$. We consider logic with equality, i.e., the set of predicate symbols contains a symbol $\approx_{S}$ for equality in every sort $S$. Usually, we will just use $\approx$ without explicitly specifying the sort. In this paper we usually assume that signatures are countable.

Terms and first-order formulae over $\Omega$ are defined in the usual way, i.e., they must respect the arities of function and predicate symbols, and the variables occurring in them are also equipped with sorts. An $\Omega$-atom is a predicate symbol applied to (sort-conforming) terms, and an $\Omega$-literal is an atom or a negated atom. A ground literal is a literal that does not contain variables. We use the notation $\phi(\underline{x})$ to express that $\phi$ is a formula whose free variables are among the 
ones in the tuple of variables $\underline{x}$. An $\Omega$-sentence is a formula over $\Omega$ without free variables. An $\Omega$-theory $T$ is a set of $\Omega$-sentences (called the axioms of $T$ ). If $T, T^{\prime}$ are $\Omega$-theories, then we write (by a slight abuse of notation) $T \subseteq T^{\prime}$ to express that all the axioms of $T$ are logical consequences of the axioms of $T^{\prime}$.

From the semantic side, we have the standard notion of an $\Omega$-structure $\mathcal{A}$, which consists of non-empty and pairwise disjoint domains $A_{S}$ for every sort $S$, and interprets function symbols $f$ and predicate symbols $P$ by functions $f \mathcal{A}$ and predicates $P^{\mathcal{A}}$ according to their arities. By $A$ (or sometimes by $|\mathcal{A}|$ ) we denote the union of all domains $A_{S}$. Validity of a formula $\phi$ in an $\Omega$-structure $\mathcal{A}(\mathcal{A} \models \phi)$, satisfiability, and logical consequence are defined in the usual way. The $\Omega$-structure $\mathcal{A}$ is a model of the $\Omega$-theory $T$ iff all axioms of $T$ are valid in $\mathcal{A}$. If $\phi(\underline{x})$ is a formula with free variables $\underline{x}=x_{1}, \ldots, x_{n}$ and $\underline{a}=a_{1}, \ldots, a_{n}$ is a (sort-conforming) tuple of elements of $A$, then we write $\mathcal{A} \models \phi(\underline{a})$ to express that $\phi(\underline{x})$ is valid in $\mathcal{A}$ under the assignment $\left\{x_{1} \mapsto a_{1}, \ldots, x_{n} \mapsto a_{n}\right\}$. Note that $\phi(\underline{x})$ is valid in $\mathcal{A}$ iff it is valid under all assignments iff its universal closure is valid in $\mathcal{A}$.

An $\Omega$-homomorphism between two $\Omega$-structures $\mathcal{A}$ and $\mathcal{B}$ is a mapping $\mu$ : $A \rightarrow B$ that is sort-conforming (i.e., maps elements of sort $S$ in $\mathcal{A}$ to elements of sort $S$ in $\mathcal{B})$, and satisfies the condition

$$
\mathcal{A} \models \alpha\left(a_{1}, \ldots, a_{n}\right) \quad \text { implies } \quad \mathcal{B} \models \alpha\left(\mu\left(a_{1}\right), \ldots, \mu\left(a_{n}\right)\right)
$$

for all $\Omega$-atoms $\alpha\left(x_{1}, \ldots, x_{n}\right)$ and (sort-conforming) elements $a_{1}, \ldots, a_{n}$ of $A$. In case the converse of (1) holds too, $\mu$ is called an embedding. Note that an embedding is something more than just an injective homomorphism since the stronger condition must hold not only for the equality predicate, but for all predicate symbols. If the embedding $\mu$ is the identity on $A$, then we say that $\mathcal{A}$ is a substructure of $\mathcal{B}$. In case (1) holds for all first order formulae, then $\mu$ is said to be an elementary embedding. If the elementary embedding $\mu$ is the identity on $A$, then we say that $\mathcal{A}$ is an elementary substructure of $\mathcal{B}$ or that $\mathcal{B}$ is an elementary extension of $\mathcal{A}$. An isomorphism is a surjective embedding, where the homomorphism $\mu: \mathcal{A} \rightarrow \mathcal{B}$ is surjective iff the restriction of $\mu$ to $A_{S}$ and $B_{S}$ is surjective for every sort $S$.

We say that $\Sigma$ is a subsignature of $\Omega$ (written $\Sigma \subseteq \Omega$ ) iff $\Sigma$ is a signature that can be obtained from $\Omega$ by removing some of its sorts and function and predicate symbols. If $\Sigma \subseteq \Omega$ and $\mathcal{A}$ is an $\Omega$-structure, then the $\Sigma$-reduct of $\mathcal{A}$ is the $\Sigma$ structure $\mathcal{A}_{\mid \Sigma}$ obtained from $\mathcal{A}$ by forgetting the interpretations of sorts, function and predicate symbols from $\Omega$ that do not belong to $\Sigma$. Conversely, $\mathcal{A}$ is called an expansion of the $\Sigma$-structure $\mathcal{A}_{\mid \Sigma}$ to the larger signature $\Omega$. If $\mu: \mathcal{A} \rightarrow \mathcal{B}$ is an $\Omega$ homomorphism, then the $\Sigma$-reduct of $\mu$ is the $\Sigma$-homomorphism $\mu_{\mid \Sigma}: \mathcal{A}_{\mid \Sigma} \rightarrow \mathcal{B}_{\mid \Sigma}$ obtained by restricting $\mu$ to the sorts that belong to $\Sigma$, i.e., by restricting the mapping to the domain of $\mathcal{A}_{\mid \Sigma}$.

Given a set $X$ of constant symbols not belonging to the signature $\Omega$, but each equipped with a sort from $\Omega$, we denote by $\Omega^{X}$ the extension of $\Omega$ by these new constants. If $\mathcal{A}$ is an $\Omega$-structure, then we can view the elements of $A$ as a set of new constants, where $a \in A_{S}$ has sort $S$. By interpreting each $a \in A$ by itself, $\mathcal{A}$ can also be viewed as an $\Omega^{A}$-structure. The positive diagram $\Delta_{\Omega}^{+}(\mathcal{A})$ of $\mathcal{A}$ is the set of all ground $\Omega^{A}$-atoms that are true in $\mathcal{A}$, the diagram $\Delta_{\Omega}(A)$ of $\mathcal{A}$ is 
the set of all ground $\Omega^{A}$-literals that are true in $\mathcal{A}$, and the elementary diagram $\Delta_{\Omega}^{e}(\mathcal{A})$ of $\mathcal{A}$ is the set of all $\Omega^{A}$-sentences that are true in $\mathcal{A}$. The subscript $\Omega$ in $\Delta_{\Omega}^{+}(\mathcal{A}), \Delta_{\Omega}(\mathcal{A})$ and $\Delta_{\Omega}^{e}(\mathcal{A})$ is sometimes omitted if there is no danger of confusion.

Robinson's diagram theorem [11] shows that there is a strong connection between diagrams and homomorphisms.

ThEOREM 2.1 (Robinson). There is a homomorphism (embedding, elementary embedding) between the $\Omega$-structures $\mathcal{A}$ and $\mathcal{B}$ iff it is possible to expand $\mathcal{B}$ to an $\Omega^{A}$-structure in such a way that it becomes a model of the positive diagram (diagram, elementary diagram) of $\mathcal{A}$.

2.2. Basic connections. In the remainder of this section, we introduce our basic scheme for connecting many-sorted theories, and illustrate it with the example of $\mathcal{E}$-connections of modal logics. Let $T_{1}, T_{2}$ be theories over the respective signatures $\Omega_{1}, \Omega_{2}$, and let $\Omega_{0}$ be a common subsignature of $\Omega_{1}$ and $\Omega_{2}$. We call $\Omega_{0}$ the connecting signature. In addition, let $T_{0}$ be an $\Omega_{0}$-theory ${ }^{1}$ that is contained in both $T_{1}$ and $T_{2}$. We define the new theory $T_{1}>_{T_{0}} T_{2}$ (called the connection of $T_{1}$ and $T_{2}$ over $\left.T_{0}\right)$ as follows.

The signature $\Omega$ of $T_{1}>_{T_{0}} T_{2}$ contains the disjoint union $\Omega_{1} \uplus \Omega_{2}$ of the signatures $\Omega_{1}$ and $\Omega_{2}$, where the shared sorts and the shared function and predicate symbols are appropriately renamed, e.g., by attaching labels 1 and 2 . Thus, if $S(f, P)$ is a sort (function symbol, predicate symbol) contained in both $\Omega_{1}$ and $\Omega_{2}$, then $S^{i}\left(f^{i}, P^{i}\right)$ for $i=1,2$ are its renamed variants in the disjoint union, where the arities are accordingly renamed. In addition, $\Omega$ contains a new function symbol $h_{S}$ of arity $S^{1} S^{2}$ for every sort $S$ of $\Omega_{0}$.

The axioms of $T_{1}>_{T_{0}} T_{2}$ are obtained as follows. Given an $\Omega_{i}$-formula $\phi$, its renamed variant $\phi^{i}$ is obtained by replacing all shared symbols by their renamed variants with label $i$. The axioms of $T_{1}>_{T_{0}} T_{2}$ consist of

$$
\left\{\phi^{1} \mid \phi \in T_{1}\right\} \cup\left\{\phi^{2} \mid \phi \in T_{2}\right\},
$$

together with the universal closures of the formulae

$$
\begin{aligned}
& h_{S}\left(f^{1}\left(x_{1}, \ldots, x_{n}\right)\right) \approx f^{2}\left(h_{S_{1}}\left(x_{1}\right), \ldots, h_{S_{n}}\left(x_{n}\right)\right), \\
& P^{1}\left(x_{1}, \ldots, x_{n}\right) \rightarrow P^{2}\left(h_{S_{1}}\left(x_{1}\right), \ldots, h_{S_{n}}\left(x_{n}\right)\right),
\end{aligned}
$$

for every function (predicate) symbol $f(P)$ in $\Omega_{0}$ of arity $S_{1} \ldots S_{n} S\left(S_{1} \ldots S_{n}\right)$.

Since the signatures $\Omega_{1}$ and $\Omega_{2}$ have been made disjoint, and since the additional axioms state that the family of mappings $h_{S}$ behaves like an $\Omega_{0}$-homomorphism, it is easy to see that the models of $T_{1}>_{T_{0}} T_{2}$ are formed by triples of the form $\left(\mathcal{M}^{1}, \mathcal{M}^{2}, h^{\mathcal{M}}\right)$, where $\mathcal{M}^{1}$ is a model of $T_{1}, \mathcal{M}^{2}$ is a model of $T_{2}$ and $h^{\mathcal{M}}$ is an $\Omega_{0}$-homomorphism

$$
h^{\mathcal{M}}: \mathcal{M}_{\mid \Omega_{0}}^{1} \rightarrow \mathcal{M}_{\mid \Omega_{0}}^{2}
$$

between the respective $\Omega_{0}$-reducts.

${ }^{1}$ When defining the connection of $T_{1}, T_{2}$, the theory $T_{0}$ is actually irrelevant; all we need is its signature $\Omega_{0}$. However, for our decidability transfer results to hold, $T_{0}$ and the $T_{i}$ must satisfy certain model-theoretic properties. 
EXAMPLE 2.2. The most basic variant of an $\mathcal{E}$-connection of modal logics [20] is an instance of our approach if one translates it into the algebraic setting. As shown in $[4,5]$, the large class of classical modal logics, which covers most of the usual modal logics, corresponds to the class of Boolean-based equational theories introduced in $[4,5]$. The theory $E$ is called Boolean-based equational theory iff its signature $\Sigma$ has just one sort, equality is the only predicate symbol, the set of function symbols contains the Boolean operators $\sqcap, \sqcup, \neg, \top, \perp$, and its set of axioms consists of identities (i.e., the universal closures of atoms $s \approx t$ ) and contains the Boolean algebra axioms.

For example, consider the basic modal logic $\mathbf{K}$, where we use only the modal operator $\diamond$ (since $\square$ can then be defined). The Boolean-based equational theory $E_{\mathbf{K}}$ corresponding to $\mathbf{K}$ is obtained from the theory of Boolean algebras by adding the identities $\diamond(x \sqcup y) \approx \diamond(x) \sqcup \diamond(y)$ and $\diamond(\perp) \approx \perp$.

We illustrate the notion of an $\mathcal{E}$-connection on this simple example (see $\S 7$ for a more general description of $\mathcal{E}$-connections and their relationship to the notion of a connection introduced in this article). To build the $\mathcal{E}$-connection of $\mathbf{K}$ with itself, one takes two disjoint copies of $\mathbf{K}$, obtained by renaming the Boolean operators and the diamonds, e.g., into $\sqcap_{i}, \sqcup_{i}, \neg_{i}, \top_{i}, \perp_{i}, \diamond_{i}$ for $i=1,2$. The signature of the $\mathcal{E}$-connection contains all these renamed symbols together with a new symbol $\diamond$. However, it is now a two-sorted signature, where symbols with index $i$ are applied to elements of sort $S_{i}$ and yield as results an element of this sort. The new symbol has arity $S_{1} S_{2}{ }^{2}$ The semantics of this $\mathcal{E}$-connection can be given in terms of Kripke structures. A Kripke structure for the $\mathcal{E}$-connection consists of two Kripke structures $\mathcal{K}_{1}, \mathcal{K}_{2}$ for $\mathbf{K}$ over disjoint domains $W_{1}$ and $W_{2}$, together with an additional connecting relation $E \subseteq W_{2} \times W_{1}$. The symbols with index $i$ are interpreted in $\mathcal{K}_{i}$, and the new symbol $\diamond$ is interpreted as the diamond operator induced by $E$, i.e., for every $X \subseteq W_{1}$ we have

$$
\diamond(X):=\left\{x \in W_{2} \mid \exists y \in W_{1} .(x, y) \in E \wedge y \in X\right\} .
$$

This interpretation of the new operator implies that it satisfies the usual identities of a diamond operator, i.e., $\diamond\left(x \sqcup_{1} y\right) \approx \diamond(x) \sqcup_{2} \diamond(y)$ and $\diamond\left(\perp_{1}\right) \approx \perp_{2}$, and that these identities are sufficient to characterize its semantics. Thus, the equational theory corresponding to the $\mathcal{E}$-connection of $\mathbf{K}$ with itself consists of these two axioms, together with the axioms of $E_{\mathbf{K}_{1}}$ and $E_{\mathbf{K}_{2}}$.

Obviously, this theory is also obtained as the connection of the theory $E_{\mathbf{K}}$ with itself, if the connecting signature $\Omega_{0}$ consists of the single sort of $E_{\mathbf{K}}$, the predicate symbol $\approx$, and the function symbols $\sqcup, \perp$. As theory $T_{0}$ we can take the theory of semilattices, i.e., the axioms that say that $\sqcup$ is associative, commutative, and idempotent, and that $\perp$ is a unit for $\sqcup$.

EXAMPLE 2.3. The previous example can be varied by additionally including $\sqcap$ in the connecting signature, and taking as theory $T_{0}$ the theory of distributive lattices with a least element $\perp$. It is easy to see that this corresponds to the case of an $\mathcal{E}$-connection where the connecting relation $E$ is required to be a partial

\footnotetext{
${ }^{2}$ In the general $\mathcal{E}$-connection scheme, there is also an inverse diamond operator $\diamond^{-}$with arity $S_{2} S_{1}$, but the algebraic approach presented in this article cannot yet treat this case (see the conclusion for a discussion).
} 
function (we call such an $\mathcal{E}$-connection deterministic). Finally, if we additionally include both $\sqcap$ and $T$ in the connecting signature, and take $T_{0}$ to be the theory of bounded distributive lattices (i.e., distributive lattices with a least and a greatest element), then the equational theory obtained through our connection corresponds to the case of an $\mathcal{E}$-connection where the connecting relation $E$ is a (total) function (we call such an $\mathcal{E}$-connection functional). More details regarding the relationship between deterministic (functional) $\mathcal{E}$-connections and connections with the theory of (bounded) distributive lattices as connecting theory can be found in $\S 7$.

2.3. The decidability transfer problem. In this paper, we are interested in deciding the universal fragments of our theories, i.e., validity of universal formulae (or, equivalently open formulae) in a theory $T$. Usually, in mathematical logic, when a first-order theory $T$ is said to be decidable, this means that one can decide whether a given elementary sentence is true in all models of $T$. However, in computer science applications, more attention is often paid to fragments of the whole elementary language: such fragments may be better behaved or more tractable, while still offering sufficient expressive power. This is, for instance, one of the main reasons of the success of Description Logics and Modal Logics in Computer Science and Artificial Intelligence applications. When using an algebraic approach, as in the present paper, the inference problems that are relevant in these applications (such as the subsumption problem in Description Logics) are well within the universal fragments of the first-order theories corresponding to these logics.

The main problem this article is concerned with is the following:

Under what conditions does decidability of the universal fragments of

$T_{1}, T_{2}$ imply decidability of the universal fragment of the connection

$T_{1}>_{T_{0}} T_{2}$ ?

In this section we show that some condition is indeed needed since there are theories whose universal fragment is decidable, but whose connection has an undecidable universal fragment.

First note that it is well-known that the problem of deciding the universal fragments of $T$ is equivalent to the problem of deciding whether a set of literals is satisfiable in some model of $T$. We call such a set of literals a constraint. By introducing new free constants (i.e., constants not occurring in the axioms of the theory), we can assume without loss of generality that such constraints are ground. In addition, we can transform any ground constraint into an equisatisfiable set of ground flat literals, i.e., literals of the form

$$
a \approx f\left(a_{1}, \ldots, a_{n}\right), \quad P\left(a_{1}, \ldots, a_{n}\right), \text { or } \neg P\left(a_{1}, \ldots, a_{n}\right),
$$

where $a, a_{1}, \ldots, a_{n}$ are (sort-conforming) free constants, $f$ is a function symbol, and $P$ is a predicate symbol (possibly also equality).

For a one-sorted first-order theory $T$, let us denote with $T^{\infty}$ the theory $T$ augmented with axioms saying that the domain of models is infinite. Obviously, $T^{\infty}$ is again a one-sorted first-order theory. The following lemma and its proof, which we give here for the sake of completeness, are taken from [10]: 
LEMma 2.4. There exists a (universal, one-sorted) first-order theory $T$ such that the universal fragment of $T$ is decidable, but the universal fragment of $T^{\infty}$ is undecidable.

Proof. Let $\Omega$ be the signature containing (in addition to the equality predicate) an infinite $\operatorname{set}^{3}$ of propositional letters $\left\{P_{(e, n)} \mid e, n \in \mathbb{N}\right\}$. We denote by $k: \mathbb{N} \times \mathbb{N} \rightarrow \mathbb{N} \cup\{\infty\}$ the function associating with each pair $(e, n)$ the number $k(e, n)$ of computation steps of the Turing Machine with index $e$ on the input $n$ (where $k(e, n):=\infty$ if the computation does not halt). Notice that the function $k(e, n)$ is not computable, but the ternary predicate $R_{k}:=\{(e, n, m) \mid k(e, n)<$ $m$ \} is decidable (because we can run the machine $e$ on input $n$ for the first $m$ steps and check whether it halts or not). The infinite (but recursive) set of axioms for $T$ is given by

$$
\left\{P_{(e, n)} \rightarrow \forall x_{1} \cdots \forall x_{m} . \bigvee_{1 \leq i<j \leq m} x_{i} \approx x_{j} \mid e, n, m \in \mathbb{N} \wedge k(e, n)<m\right\}
$$

These axioms say that, if the Turing Machine $e$ halts in less than $m$ steps on input $n$, then the cardinality of the domains of models of $T$ in which $P_{(e, n)}$ is true is smaller than $m$.

To show that the universal fragment of $T$ is decidable, consider a constraint $\Gamma$ consisting of ground flat literals over a suitably expanded signature $\Omega \underline{a}$, where $\underline{a}$ is a finite set of free constant symbols. First, guess a set of literals $\Gamma_{0}$ containing, for every $a, a^{\prime} \in \underline{a}$, either $a \approx a^{\prime}$ or $a \not z a^{\prime}$. Clearly, $\Gamma$ is satisfiable in a model of $T$ iff there is such a set $\Gamma_{0}$ such that $\Gamma \cup \Gamma_{0}$ is satisfiable in a model of $T$. Then, check the set of equations and inequations from $\Gamma \cup \Gamma_{0}$ for satisfiability in the pure theory of equality, which is known to be decidable. Suppose the satisfiability check succeeds; let $m$ be the number of equivalence classes for the equivalence relation $\equiv$ over $\underline{a}$ induced by the equations in $\Gamma_{0}$, i.e., $a \equiv a^{\prime}$ iff $a \approx a^{\prime} \in \Gamma_{0}$. Note that this is indeed an equivalence relation since we have $a \not a^{\prime} \in \Gamma_{0}$ whenever $a \approx a^{\prime} \notin \Gamma_{0}$, and the satisfiability test succeeded. Then $m$ is the minimal cardinality of a candidate model for $\Gamma \cup \Gamma_{0}$. Clearly, $\Gamma \cup \Gamma_{0}$ is unsatisfiable if it contains both $P_{(e, n)}$ and $\neg P_{(e, n)}$ for some $e, n \in \mathbb{N}$. Assume that this is not the case. We claim that

$$
\Gamma \cup \Gamma_{0} \text { is satisfiable iff } P_{(e, n)} \in \Gamma \text { implies } m \leq k(e, n) .
$$

Note that the condition on the right-hand side of the iff-statement is decidable since the predicate $R_{k}$ is decidable. Thus, to show that the universal fragment of $T$ is decidable, it remains to prove the claim.

First, assume that $\Gamma \cup \Gamma_{0}$ is satisfiable and that $P_{(e, n)} \in \Gamma$. The cardinality of the model of $T$ satisfying $\Gamma \cup \Gamma_{0}$ is at least $m$, and thus the definition of $T$ implies that we cannot have $k(e, n)<m$. Conversely, assume that $P_{(e, n)} \in \Gamma$ implies $m \leq k(e, n)$. We consider the structure $\mathcal{M}$ of cardinality $m$ that has the equivalence classes of $\equiv$ as elements, interprets the constants from $\underline{a}$ by their respective class, and makes the predicates $P_{(e, n)}$ true if they belong to $\Gamma$. Obviously, $\mathcal{M}$ satisfies the equations and inequations from $\Gamma \cup \Gamma_{0}$, and it also

\footnotetext{
${ }^{3}$ Thus, $\Omega$ is an infinite signature. By modifying the definition of the theory $T$ given in $(2)$, it is also possible to prove the lemma for a theory over a finite signature (see [10]).
} 
satisfies all the literals in $\Gamma$ involving the predicates $P_{(e, n)}$. It remains to show that it is a model of $T$. Thus, consider an axiom of the form

$$
P_{(e, n)} \rightarrow \forall x_{1} \cdots \forall x_{\mu} . \bigvee_{1 \leq i<j \leq \mu} x_{i} \approx x_{j} \quad \text { where } k(e, n)<\mu
$$

and assume that $P_{(e, n)}$ is true in $\mathcal{M}$. By the definition of $\mathcal{M}$, this implies that $P_{(e, n)} \in \Gamma$, and thus our assumption yields $m \leq k(e, n)<\mu$. Since $\mathcal{M}$ has cardinality $m$, this shows that the above axiom is true in $\mathcal{M}$.

To see that $T^{\infty}$ does not have a decidable universal fragment, notice that the atom $P_{(e, n)}$ is satisfiable in a model of $T^{\infty}$ iff $k(e, n)=\infty$, i.e. iff the Turing Machine with index $e$ does not halt on input $n$. The latter is obviously undecidable since it is the complement of the Halting problem.

To show that decidability need not transfer to the connection, we use a construction that will turn out to be useful also later on. Let $T$ be a theory over the signature $\Omega$. We build the signature $\Omega\urcorner$ and the theory $T\urcorner$ as follows: for every $n$-ary predicate symbol $P$ of $\Omega$, the signature $\Omega\urcorner$ extends $\Omega$ by the additional new $n$-ary predicate symbol $P\urcorner$, and the theory $T\urcorner$ contains in addition to the axioms of $T$ the axioms

$$
\neg P\left(x_{1}, \ldots, x_{n}\right) \leftrightarrow P \neg\left(x_{1}, \ldots, x_{n}\right),
$$

which define each $P\urcorner$ as the negation of $P$.

The difference between $T$ and $T\urcorner$ is not relevant at the level of models: every model of $T$ can be expanded in a unique way to a model of $T\urcorner$. It is also not relevant w.r.t. the decidability of the universal theory: every $\Omega\urcorner$-constraint can obviously be transformed into an equi-satisfiable $\Omega$-constraint by replacing $P\urcorner$ by $\neg P$, and every $\Omega$-constraint is satisfiable in $T$ iff it is satisfiable in $T\urcorner$. However, there is a remarkable difference at the level of homomorphisms: a homomorphism between models of $T\urcorner$ is just an embedding between the corresponding models of $T$. This difference is exploited in the proof of the main result of this section.

THEOREM 2.5. There exists theories $T_{0}, T_{1}, T_{2}$ such that $T_{0}$ is contained in $T_{1}$ and $T_{2}$ and the universal fragments of $T_{0}, T_{1}, T_{2}$ are decidable, but the universal fragment of $T_{1}>_{T_{0}} T_{2}$ is undecidable.

Proof. Let $T_{0}^{\prime}$ be the (one-sorted) theory of pure equality, i.e., the theory whose signature contains no function symbols and equality as the only predicate symbol, and which is axiomatized by the empty set. It is well known that the universal fragment of $T_{0}^{\prime}$ is decidable. Let $T_{1}^{\prime}$ be a theory having only infinite models and a decidable universal fragment, ${ }^{4}$ and let $T_{2}^{\prime}$ be a theory satisfying the conditions of Lemma 2.4, i.e., $T_{2}^{\prime}$ is one sorted, the universal fragment of $T_{2}^{\prime}$ is decidable, but the universal fragment of $T_{2}^{\prime \infty}$ is not decidable. Let us consider the theories $\left.\left.T_{0}:=T_{0}^{\prime}\right\urcorner, T_{1}:=T_{1}^{\prime}\right\urcorner$, and $\left.T_{2}:=T_{2}^{\prime}\right\urcorner$. Clearly, the universal fragments of these theories are also decidable, and $T_{0}$ is contained in $T_{1}$ and $T_{2}$. Models of $T_{1}>_{T_{0}} T_{2}$ are now formed by triples of the form $\left(\mathcal{M}^{1}, \mathcal{M}^{2}, h\right)$, where $\mathcal{M}^{1}$ is a model of $T_{1}, \mathcal{M}^{2}$ is a model of $T_{2}$, and

$$
h: M^{1} \rightarrow M^{2}
$$

\footnotetext{
${ }^{4}$ There are many theories satisfying this requirement, e.g., Presburger Arithmetic [27] or the theory of acyclic lists [25].
} 
is an injective function. Consider now a $T_{1}>_{T_{0}} T_{2}$-constraint $\Gamma$ that consists of $\Sigma_{2}$-literals: such a constraints is $T_{1}>_{T_{0}} T_{2}$-satisfiable iff it is satisfiable in an infinite model of $T_{2}$, and thus in an infinite model of $T_{2}^{\prime}$. The latter problem is undecidable by our choice of $T_{2}^{\prime}$.

We shall comment on the peculiarities of the theories introduced in the proof of Theorem 2.5 in more detail in Example 4.10 below.

$\S 3$. Positive-existential model completions and compatibility. In order to transfer decidability results from the component theories $T_{1}, T_{2}$ to their connection $T_{1}>_{T_{0}} T_{2}$ over $T_{0}$, the theories $T_{0}, T_{1}, T_{2}$ must satisfy certain modeltheoretic conditions, which we introduce below. The most important one is that $T_{0}$ has a positive-existential model completion. ${ }^{5}$ Before we can define this concept, we must introduce some notions from model theory.

The formula $\phi$ is called open iff it does not contain quantifiers; it is called universal iff it is obtained from an open formula by adding a prefix of universal quantifiers; and it is called geometric iff it is built from atoms by using conjunction, disjunction, and existential quantifiers. The latter formulae are called "geometric" in categorical logic [21] since they are preserved under inverse image geometric morphisms among toposes.

The main property of geometric formulae is that they are preserved under homomorphisms in the following sense: if $\mu: \mathcal{A} \rightarrow \mathcal{B}$ is a homomorphism between $\Omega$-structures and $\phi\left(x_{1}, \ldots, x_{n}\right)$ is a geometric formula over $\Omega$, then

$$
\mathcal{A}=\phi\left(a_{1}, \ldots, a_{n}\right) \quad \text { implies } \quad \mathcal{B} \models \phi\left(\mu\left(a_{1}\right), \ldots, \mu\left(a_{n}\right)\right)
$$

for all (sort-conforming) $a_{1}, \ldots, a_{n} \in A$.

Open formulae are related to embeddings in various way. First, they are preserved under building sub- and superstructures, i.e., if $\mathcal{A}$ is a substructure of $\mathcal{B}, \phi\left(x_{1}, \ldots, x_{n}\right)$ is an open formula, and $a_{1}, \ldots, a_{n} \in A$ are sort-conforming, then $\mathcal{A} \models \phi\left(a_{1}, \ldots, a_{n}\right)$ iff $\mathcal{B} \models \phi\left(a_{1}, \ldots, a_{n}\right)$. The following lemma is wellknown [11]:

LEMmA 3.1. Two $\Omega$-theories $T, T^{\prime}$ entail the same set of open formulae iff every model of $T$ can be embedded into a model of $T^{\prime}$ and vice versa.

Since a theory entails an open formula iff it entails its universal closure, the lemma also says that two theories $T, T^{\prime}$ entail the same universal sentences iff every model of $T$ can be embedded into a model of $T^{\prime}$ and vice versa.

The theory $T$ is a universal theory iff its axioms are universal sentences; it is a geometric theory iff it can be axiomatized by using universal closures of geometric sequents, where a geometric sequent is an implication between two geometric formulae. Note that any universal theory is geometric since open formulae are conjunctions of clauses and clauses can be rewritten as geometric sequents.

\footnotetext{
${ }^{5}$ In the conference version of this article [3], we used the name "positive algebraic completion." However, when preparing the present extended version, we changed this and other names (e.g., the "positive co-algebraic completions" of [3] are now called "positive-universal model completions"). We think that these new names are more intuitive.
} 
Definition 3.2. Let $T$ be a universal and $T^{*}$ a geometric theory over $\Omega$. We say that $T^{*}$ is a positive-existential model completion of $T$ iff the following properties hold:

1. $T \subseteq T^{*}$;

2. every model of $T$ embeds into a model of $T^{*} ; 6$

3. for every geometric formula $\phi(\underline{x})$ there is an open geometric formula $\phi^{*}(\underline{x})$ such that $T^{*} \models \phi \leftrightarrow \phi^{*}$.

It can be shown that the models of $T^{*}$ are exactly the positive-existentially closed models of $T{ }^{7}$ In particular, this means that the positive-existential model completion of $T$ is unique, provided that it exists.

When trying to show that Property 3 of Definition 3.2 holds for given theories $T, T^{*}$, then it is sufficient to consider simple existential formulae $\phi(\underline{x})$, i.e., formulae that are obtained from conjunctions of atoms by adding an existential quantifier prefix. In fact, any geometric formula $\phi$ can be normalized to a disjunction $\phi_{1} \vee \ldots \vee \phi_{n}$ of simple existential formulae $\phi_{i}$ by using distributivity of conjunction and existential quantification over disjunction. In addition, if $T^{*} \models \phi_{i} \leftrightarrow \phi_{i}^{*}$ for geometric open formulae $\phi_{i}^{*}(i=1, \ldots, n)$, then $\phi_{1}^{*} \vee \ldots \vee \phi_{n}^{*}$ is also a geometric open formula and $T^{*} \models\left(\phi_{1} \vee \ldots \vee \phi_{n}\right) \leftrightarrow\left(\phi_{1}^{*} \vee \ldots \vee \phi_{n}^{*}\right)$.

The following lemma will turn out to be useful later on.

Lemma 3.3. Assume that T, $T^{*}$ satisfy Property 1 and 2 of Definition 3.2. If $\phi(\underline{x})$ is a simple existential formula and $\phi^{*}(\underline{x})$ is an open formula, then $T^{*}=$ $\phi \rightarrow \phi^{*}$ iff $T=\phi \rightarrow \phi^{*}$.

This is an immediate consequence of the fact that $\phi \rightarrow \phi^{*}$ is then equivalent to an open formula, and hence Lemma 3.1 applies.

The first ingredient of our combinability condition is the following notion of compatibility, which is a variant of analogous compatibility conditions introduced in $[16,4,5]$ for the case of the union of theories.

Definition 3.4. Let $T_{0} \subseteq T$ be theories over the respective signatures $\Omega_{0} \subseteq$ $\Omega_{1}$. We say that $T$ is $T_{0}$-positive-existentially compatible iff $T_{0}$ is universal, has a positive-existential model completion $T_{0}^{*}$, and every model of $T$ embeds into a model of $T \cup T_{0}^{*}$.

The second ingredient ensures that all finitely generated models of $T_{0}$ are finite. We adopt the following effective variant of a condition known as local finiteness (see e.g. $[16,4,5]$ ).

DEFinition 3.5. Let $T_{0}$ be a universal theory over the finite signature $\Omega_{0}$. Then $T_{0}$ is called effectively locally finite iff $\Omega_{0}$ is finite and, for every tuple of variables $\underline{x}$, one can effectively determine terms $t_{1}(\underline{x}), \ldots, t_{k}(\underline{x})$ such that, for every further term $u(\underline{x})$, we have that $T_{0}=u \approx t_{i}$ for some $i=1, \ldots, k$.

\footnotetext{
${ }^{6}$ Equivalently, $T$ and $T^{*}$ entail the same universal sentences.

${ }^{7}$ See $\S 8$ for a definition of positive-existentially closed models, and proofs of this and other model-theoretic properties of positive-existential model completions.
} 
\$4. The main combination results. Recall that we are interested in deciding the universal fragments of our theories, i.e., validity of universal formulae (or, equivalently open formulae) in a theory $T{ }^{8}$ As shown in Section 2.3, we can restrict the attention to deciding the satisfiability of constraints consisting of ground flat literals, i.e., literals of the form

$$
a \approx f\left(a_{1}, \ldots, a_{n}\right), \quad P\left(a_{1}, \ldots, a_{n}\right), \text { or } \neg P\left(a_{1}, \ldots, a_{n}\right),
$$

where $a, a_{1}, \ldots, a_{n}$ are (sort-conforming) free constants, $f$ is a function symbol, and $P$ is a predicate symbol (possibly also equality).

In the following, we first treat the case of a basic connection, as introduced in $\S 2$. Then, we show that the combination result can be extended to connections with several connection functions, possibly going in both directions. Finally, we give examples of theories satisfying our combinability conditions.

4.1. Basic connections. In this subsection, we provide sufficient conditions under which decidability of the universal fragments of $T_{1}, T_{2}$ transfers to their connection $T_{1}>_{T_{0}} T_{2}$.

THEOREM 4.1. Let $T_{0}, T_{1}$, and $T_{2}$ be theories over the respective signatures $\Omega_{0}, \Omega_{1}$, and $\Omega_{2}$, where $\Omega_{0}$ is a common subsignature of $\Omega_{1}$ and $\Omega_{2}$. Assume that $T_{0} \subseteq T_{1}$ and $T_{0} \subseteq T_{2}$, that $T_{0}$ is universal and locally finite, and that $T_{2}$ is $T_{0}$ positive-existentially compatible. Then the decidability of the universal fragments of $T_{1}$ and $T_{2}$ entails the decidability of the universal fragment of $T_{1}>_{T_{0}} T_{2}$.

To prove the theorem, we consider a finite set $\Gamma$ of ground flat literals over the signature $\Omega$ of $T_{1}>_{T_{0}} T_{2}$ (with additional free constants), and show how it can be tested for satisfiability in $T_{1}>_{T_{0}} T_{2}$. Since all literals in $\Gamma$ are flat, we can divide $\Gamma$ into three disjoint sets $\Gamma=\Gamma_{0} \cup \Gamma_{1} \cup \Gamma_{2}$, where $\Gamma_{i}(i=1,2)$ is a set of literals in the signature $\Omega_{i}$ (expanded with free constants), and $\Gamma_{0}$ is of the form

$$
\Gamma_{0}=\left\{h\left(a_{1}\right) \approx b_{1}, \ldots, h\left(a_{n}\right) \approx b_{n}\right\}
$$

for free constants $a_{1}, b_{1}, \ldots, a_{n}, b_{n}$.

The next proposition will allow us to specify the decision procedure for $T_{1}>_{T_{0}}$ $T_{2}$ required by Theorem 4.1 .

Proposition 4.2. The constraint $\Gamma=\Gamma_{0} \cup \Gamma_{1} \cup \Gamma_{2}$ is satisfiable in $T_{1}>_{T_{0}} T_{2}$ iff there exists a triple $(\mathcal{A}, \mathcal{B}, \nu)$ such that

1. $\mathcal{A}$ is an $\Omega_{0}$-model of $T_{0}$, which is generated by $\left\{a_{1}^{\mathcal{A}}, \ldots, a_{n}^{\mathcal{A}}\right\}$;

2. $\mathcal{B}$ is an $\Omega_{0}$-model of $T_{0}$, which is generated by $\left\{b_{1}^{\mathcal{B}}, \ldots, b_{n}^{\mathcal{B}}\right\}$;

3. $\nu: \mathcal{A} \rightarrow \mathcal{B}$ is an $\Omega_{0}$-homomorphism such that $\nu\left(a_{j}^{\mathcal{A}}\right)=b_{j}^{\mathcal{B}}$ for $j=1, \ldots, n$;

4. $\Gamma_{1} \cup \Delta_{\Omega_{0}}(\mathcal{A})$ is satisfiable in $T_{1}$;

5. $\Gamma_{2} \cup \Delta_{\Omega_{0}}(\mathcal{B})$ is satisfiable in $T_{2}$.

Proof. The only-if direction is simple. In fact, as noted in $\S 2$, a model $\mathcal{M}$ of $T_{1}>_{T_{0}} T_{2}$ is given by a triple $\left(\mathcal{M}^{1}, \mathcal{M}^{2}, h^{\mathcal{M}}\right)$, where $\mathcal{M}^{1}$ is a model of $T_{1}, \mathcal{M}^{2}$ is a model of $T_{2}$ and $h^{\mathcal{M}}: \mathcal{M}_{\mid \Omega_{0}}^{1} \rightarrow \mathcal{M}_{\mid \Omega_{0}}^{2}$ is an $\Omega_{0}$-homomorphism between the respective $\Omega_{0}$-reducts. Assume that this model $\mathcal{M}$ satisfies $\Gamma$. We can take as $\mathcal{A}$ the substructure of $\mathcal{M}_{\mid \Omega_{0}}^{1}$ generated by (the interpretations of) $a_{1}, \ldots, a_{n}$, as

\footnotetext{
${ }^{8}$ This is the decision problem also treated by the Nelson-Oppen combination method, albeit
} for the union of theories. 
$\mathcal{B}$ the substructure of $\mathcal{M}_{\mid \Omega_{0}}^{2}$ generated by (the interpretations of) $b_{1}, \ldots, b_{n}$, and as homomorphism $\nu$ the restriction of $h^{\mathcal{M}}$ to $\mathcal{A}$. It is easy to see that the triple $(\mathcal{A}, \mathcal{B}, \nu)$ obtained this way satisfies $1 .-5$. of the proposition.

Conversely, assume that $(\mathcal{A}, \mathcal{B}, \nu)$ is a triple satisfying $1 .-5$. of the proposition. Because of 4 . and 5 , there is an $\Omega_{1}$-model $\mathcal{N}^{\prime}$ of $T_{1}$ satisfying $\Gamma_{1} \cup \Delta_{\Omega_{0}}(\mathcal{A})$ and an $\Omega_{2}$-model $\mathcal{N}^{\prime \prime}$ of $T_{2}$ satisfying $\Gamma_{2} \cup \Delta_{\Omega_{0}}(\mathcal{B})$. By Robinson's diagram theorem, $\mathcal{N}^{\prime}$ has $\mathcal{A}$ as an $\Omega_{0}$-substructure and $\mathcal{N}^{\prime \prime}$ has $\mathcal{B}$ as an $\Omega_{0}$-substructure. We assume without loss of generality that $\mathcal{N}^{\prime}$ is at most countable and that $\mathcal{N}^{\prime \prime}$ is a model of $T_{2} \cup T_{0}^{*}$. The latter assumption is by $T_{0}$-positive-existential compatibility of $T_{2}$, and the former assumption is by the Löwenheim-Skolem theorem since our signatures are at most countable. Let us enumerate the elements of $\mathcal{N}^{\prime}$ as

$$
c_{1}, c_{2}, \ldots, c_{n}, c_{n+1}, \ldots
$$

where we assume that $c_{i}=a_{i}^{\mathcal{A}}(i=1, \ldots, n)$, i.e., $c_{1}, \ldots, c_{n}$ are generators of $\mathcal{A}$. We define an increasing sequence of sort-conforming functions $\nu_{k}:\left\{c_{1}, \ldots c_{k}\right\} \rightarrow$ $N^{\prime \prime}$ (for $k \geq n$ ) such that, for every ground $\Omega_{0}^{\left\{c_{1}, \ldots, c_{k}\right\}}$-atom $\alpha$ we have

$$
\mathcal{N}_{\mid \Omega_{0}}^{\prime} \models \alpha\left(c_{1}, \ldots, c_{k}\right) \quad \text { implies } \quad \mathcal{N}_{\mid \Omega_{0}}^{\prime \prime} \models \alpha\left(\nu_{k}\left(c_{1}\right), \ldots, \nu_{k}\left(c_{k}\right)\right) .
$$

We first take $\nu_{n}$ to be $\nu$. To define $\nu_{k+1}$ (for $k \geq n$ ), let us consider the conjunction $\psi\left(c_{1}, \ldots, c_{n}, c_{n+1}\right)$ of the $\Omega_{0}^{\left\{c_{1}, \ldots, c_{n+1}\right\}}$-atoms that are true in $\mathcal{N}_{\mid \Omega_{0}}^{\prime}$ : this conjunction is finite (modulo taking representative terms, thanks to local finiteness of $\left.T_{0}\right)$. Let $\phi\left(x_{1}, \ldots, x_{n}\right)$ be $\exists x_{n+1} . \psi\left(x_{1}, \ldots, x_{n}, x_{n+1}\right)$ and let $\phi^{*}\left(x_{1}, \ldots, x_{n}\right)$ be a geometric open formula such that $T_{0}^{*} \models \phi \leftrightarrow \phi^{*}$.

By Lemma 3.3, $T_{0} \models \phi \rightarrow \phi^{*}$, and thus we have $\mathcal{N}_{\mid \Omega_{0}}^{\prime} \models \phi^{*}\left(c_{1}, \ldots, c_{k}\right)$ and also $\mathcal{N}_{\mid \Omega_{0}}^{\prime \prime} \models \phi^{*}\left(\nu_{k}\left(c_{1}\right), \ldots, \nu_{k}\left(c_{k}\right)\right)$ by the induction hypothesis. Since $\mathcal{N}_{\mid \Omega_{0}}^{\prime \prime}$ is a model of $T_{0}^{*}$, there is a $b$ such that $\mathcal{N}_{\mid \Omega_{0}}^{\prime \prime} \models \psi\left(\nu_{k}\left(c_{1}\right), \ldots, \nu_{k}\left(c_{k}\right), b\right)$ for some $b$. We now obtain the desired extension $\nu_{k+1}$ of $\nu_{k}$ by setting $\nu_{k+1}\left(c_{k+1}\right):=b$. Taking $\nu_{\infty}=\bigcup_{k \geq n} \nu_{k}$, we finally obtain a homomorphism $\nu_{\infty}: \mathcal{N}_{\mid \Omega_{0}}^{\prime} \rightarrow \mathcal{N}_{\mid \Omega_{0}}^{\prime \prime}$ such that the triple $\left(\mathcal{N}^{\prime}, \mathcal{N}^{\prime \prime}, \nu_{\infty}\right)$ is a model of $T_{1}>_{T_{0}} T_{2}$ that satisfies $\Gamma_{0} \cup \Gamma_{1} \cup \Gamma_{2}$. $\dashv$

The above proof uses the assumption that $T_{0}$ is locally finite. By using heavier model-theoretic machinery, one can also prove the proposition without using local finiteness of $T_{0}$ (see $\S 9$ ). However, since the proof of Theorem 4.1 needs this assumption anyway (see below), we gave the above proof since it is simpler.

We can now conclude the proof of Theorem 4.1:

PROOF. Let us describe a non-deterministic decision procedure that effectively guesses an appropriate triple $(\mathcal{A}, \mathcal{B}, \nu)$ and then checks whether it satisfies 1.-5. of Proposition 4.2. To guess an $\Omega_{0}$-model of $T_{0}$ that is generated by a finite set $X$, one uses effective local finiteness of $T_{0}$ to obtain an effective bound on the size of such a model and guesses an $\Omega_{0}$-structure that satisfies this size bound.

Once the $\Omega_{0}$-structures $\mathcal{A}, \mathcal{B}$ are given, one can build their diagrams, and use the decision procedures for $T_{1}$ and $T_{2}$ to check whether 4 . and 5 . of Proposition 4.2 are satisfied. If the answer is yes, then $\mathcal{A}, \mathcal{B}$ are also models of $T_{0}$ : in fact, if for instance $\Gamma_{1} \cup \Delta_{\Omega_{0}}(\mathcal{A})$ is satisfiable in the model $\mathcal{M}$ of $T_{1}$, then $\mathcal{M}$ has $\mathcal{A}$ as a substructure, and this implies $\mathcal{A} \models T_{0}$ because $T_{0}$ is universal and $T_{0} \subseteq T_{1}$. 
Finally, one can guess a mapping $\nu: A \rightarrow B$ that satisfies $\nu\left(a_{j}^{\mathcal{A}}\right)=b_{j}^{\mathcal{B}}$, and then use the diagrams of $\mathcal{A}, \mathcal{B}$ to check whether $\nu$ satisfies the homomorphism condition (1).

4.2. Two-side connections. The proof of Proposition 4.2 basically shows that our decidability transfer result can easily be extended to the case of several connection functions, possibly going in both directions. For simplicity, we examine only the case of two connection functions, going in the two opposite directions.

The theory $T_{1}>_{T_{0}}<T_{2}$ is defined as the union of $T_{1}>_{T_{0}} T_{2}$ and $T_{2}>_{T_{0}} T_{1}$. Thus, a model of $T_{1}>_{T_{0}}<T_{2}$ is a 4 -tuple given by a model $\mathcal{M}^{1}$ of $T_{1}$, a model $\mathcal{M}^{2}$ of $T_{2}$ and two homomorphisms

$$
h^{\mathcal{M}}: \mathcal{M}_{\mid \Omega_{0}}^{1} \rightarrow \mathcal{M}_{\mid \Omega_{0}}^{2} \quad \text { and } \quad g^{\mathcal{M}}: \mathcal{M}_{\mid \Omega_{0}}^{2} \rightarrow \mathcal{M}_{\mid \Omega_{0}}^{1}
$$

among the respective $\Omega_{0}$-reducts.

THEOREM 4.3. Let $T_{0}, T_{1}$, and $T_{2}$ be theories over the respective signatures $\Omega_{0}, \Omega_{1}$, and $\Omega_{2}$, where $\Omega_{0}$ is a common subsignature of $\Omega_{1}$ and $\Omega_{2}$. Assume that $T_{0} \subseteq T_{1}$ and $T_{0} \subseteq T_{2}$, that $T_{0}$ is universal and locally finite, and that $T_{1}, T_{2}$ are both $T_{0}$-positive-existentially compatible. Then the decidability of the universal fragments of $T_{1}$ and $T_{2}$ entails the decidability of the universal fragment of $T_{1}>_{T_{0}}<T_{2}$.

To prove the theorem, notice that any finite set of ground flat literals (with free constants) $\Gamma$ to be tested for $T_{1}>_{T_{0}}<T_{2}$-consistency can be divided into four disjoint sets

$$
\Gamma=\Theta_{1} \cup \Theta_{2} \cup \Gamma_{1} \cup \Gamma_{2},
$$

where $\Gamma_{i}(i=1,2)$ are sets of literals in the signature $\Omega_{i}$ (expanded with free constants), and

$$
\Theta_{1}=\left\{h\left(a_{1}\right) \approx b_{1}, \ldots, h\left(a_{n}\right) \approx b_{n}\right\} \text { and } \Theta_{2}=\left\{g\left(b_{1}^{\prime}\right) \approx a_{1}^{\prime}, \ldots, g\left(b_{m}^{\prime}\right) \approx a_{m}^{\prime}\right\} .
$$

Theorem 4.3 is an easy consequence of the following proposition.

Proposition 4.4. The constraint $\Gamma=\Theta_{1} \cup \Theta_{2} \cup \Gamma_{1} \cup \Gamma_{2}$ is satisfiable in $T_{1}>_{T_{0}}<T_{2}$ iff there exist two triples $(\mathcal{A}, \mathcal{B}, \nu)$ and $\left(\mathcal{A}^{\prime}, \mathcal{B}^{\prime}, \nu^{\prime}\right)$ such that

1. $\mathcal{A}$ is a $\Omega_{0}$-model of $T_{0}$ that is generated by $\left\{a_{1}^{\mathcal{A}}, \ldots, a_{n}^{\mathcal{A}}\right\}, \mathcal{B}$ is a $\Omega_{0}$-model of $T_{0}$ which is generated by $\left\{b_{1}^{\mathcal{B}}, \ldots, b_{n}^{\mathcal{B}}\right\}$ and $\nu: \mathcal{A} \rightarrow \mathcal{B}$ is a $\Omega_{0}$-homomorphism such that $\nu\left(a_{j}^{\mathcal{A}}\right)=b_{j}^{\mathcal{B}}$ for all $j=1, \ldots, n$;

2. $\mathcal{A}^{\prime}$ is a $\Omega_{0}$-model of $T_{0}$ that is generated by $\left\{a_{1}^{\prime \mathcal{A}^{\prime}}, \ldots, a_{m}^{\prime}{ }^{\mathcal{A}^{\prime}}\right\}, \mathcal{B}^{\prime}$ is a $\Omega_{0^{-}}$ model of $T_{0}$ that is generated by $\left\{b_{1}^{\prime \mathcal{B}^{\prime}}, \ldots, b_{m}^{\prime}{ }^{\mathcal{B}^{\prime}}\right\}$ and $\mu: \mathcal{B}^{\prime} \rightarrow \mathcal{A}^{\prime}$ is a $\Omega_{0}$-homomorphism such that $\nu^{\prime}\left({b_{j}^{\prime}}^{\mathcal{B}^{\prime}}\right)=a_{j}^{\prime \mathcal{A}^{\prime}}$ for all $j=1, \ldots, m$;

3. $\Gamma_{1} \cup \Delta_{\Omega_{0}}(\mathcal{A}) \cup \Delta_{\Omega_{0}}\left(\mathcal{A}^{\prime}\right)$ is satisfiable in $T_{1}$, and $\Gamma_{2} \cup \Delta_{\Omega_{0}}(\mathcal{B}) \cup \Delta_{\Omega_{0}}\left(\mathcal{B}^{\prime}\right)$ is satisfiable in $T_{2}$.

Proof. The only-if direction is again simple. To prove the if direction, assume that for some $\nu: \mathcal{A} \rightarrow \mathcal{B}$ and $\mu: \mathcal{B}^{\prime} \rightarrow \mathcal{A}^{\prime}$, the set of literals $\Gamma_{1} \cup \Delta_{\Omega_{0}}(\mathcal{A}) \cup \Delta_{\Omega_{0}}\left(\mathcal{A}^{\prime}\right)$ is satisfiable in an $\Omega_{1}$-model $\mathcal{N}^{\prime}$ of $T_{1}$, and the set of literals $\Gamma_{2} \cup \Delta_{\Omega_{0}}(\mathcal{B}) \cup \Delta_{\Omega_{0}}\left(\mathcal{B}^{\prime}\right)$ is satisfiable in an $\Omega_{2}$-model $\mathcal{N}^{\prime \prime}$ of $T_{2}$. By Robinson's diagram theorem, $\mathcal{N}^{\prime}$ has $\mathcal{A}$ and $\mathcal{A}^{\prime}$ as $\Omega_{0}$-substructures, and $\mathcal{N}^{\prime \prime}$ has $\mathcal{B}$ and $\mathcal{B}^{\prime}$ as $\Omega_{0}$-substructures. We 
assume without loss of generality that $\mathcal{N}^{\prime}$ and $\mathcal{N}^{\prime \prime}$ are at most countable models of $T_{1} \cup T_{0}^{*}$ and $T_{1} \cup T_{0}^{*}$, respectively.

Now, an argument identical to the one used in the proof of Proposition 4.2 yields the homomorphisms

$$
\nu_{\infty}: \mathcal{N}_{\mid \Omega_{0}}^{\prime} \rightarrow \mathcal{N}_{\mid \Omega_{0}}^{\prime \prime} \quad \text { and } \quad \nu_{\infty}^{\prime}: \mathcal{N}_{\mid \Omega_{0}}^{\prime \prime} \rightarrow \mathcal{N}_{\mid \Omega_{0}}^{\prime},
$$

which are needed in order to obtain a full model of $T_{1}>_{T_{0}}<T_{2}$.

It should be clear how to adapt this proof to the case of more than one connection function going in each direction.

4.3. Examples. When trying to axiomatize the positive-existential model completion $T_{0}^{*}$ of a given universal theory $T_{0}$, it is sufficient to produce for every simple existential formula $\phi(\underline{x})$ an appropriate geometric and open formula $\phi^{*}(\underline{x})$. Take as theory $T_{0}^{*}$ the one axiomatized by $T_{0}$ together with the formulae $\phi \leftrightarrow \phi^{*}$ for every simple existential formula $\phi$. In order to complete the job, it is sufficient to show that every model of $T_{0}$ embeds into a model of $T_{0}^{*}$. It should also be noted that one can without loss of generality restrict the attention to simple existential formulae with just one existential quantifier since more than one quantifier can then be treated by iterated elimination of single quantifiers.

In the next example we encounter a special case where the formulae $\phi \leftrightarrow \phi^{*}$ are already valid in $T_{0}$. In this case, we have $T_{0}=T_{0}^{*}$, and thus the modelembedding condition is trivially satisfied. In addition, any theory $T$ with $T_{0} \subseteq T$ is $T_{0}$-positive-existentially compatible.

EXAmPLE 4.5. Recall from $[4,5]$ the definition of a Gaussian theory. Let us call a conjunction of atoms an e-formula. The universal theory $T_{0}$ is Gaussian iff for every $e$-formula $\phi(\underline{x}, y)$ it is possible to compute an $e$-formula $\psi(\underline{x})$ and a term $s(\underline{x}, \underline{z})$ with fresh variables $\underline{z}$ such that

$$
T_{0} \models \phi(\underline{x}, y) \leftrightarrow(\psi(\underline{x}) \wedge \exists \underline{z} \cdot(y \approx s(\underline{x}, \underline{z}))) .
$$

Any Gaussian theory $T_{0}$ is its own positive-existential model completion. In fact, it is easy to see that $(3)$ implies $T_{0}=(\exists y . \phi(\underline{x}, y)) \leftrightarrow \psi(\underline{x})$, and thus $T_{0}=T_{0}^{*}$.

As a consequence, our combination result applies to all the examples of effectively locally finite Gaussian theories given in $[4,5]$ (e.g., Boolean algebras, vector spaces over a finite field, empty theory over a signature whose sets of predicates consists of $\approx$ and whose set of function symbols is empty): if the universal theory $T_{0}$ is effectively locally finite and Gaussian, and $T_{1}, T_{2}$ are arbitrary theories containing $T_{0}$ and with decidable universal fragment, then the universal fragment of $T_{1}>_{T_{0}} T_{2}$ is also decidable.

EXAMPLE 4.6. Let $T_{0}$ be the theory of semilattices (see Example 2.2). This theory is obviously effectively locally finite. In the following, we use the disequation $s \sqsubseteq t$ as an abbreviation for the equation $s \sqcup t \approx t$. Obviously, any equation $s \approx t$ can be expressed by the disequations $s \sqsubseteq t \wedge t \sqsubseteq s$.

The theory $T_{0}$ has a positive-existential model completion, which can be axiomatized as follows. Let $\phi(\underline{x})$ be a simple existential formula with just one existential quantifier. Using the fact that $z_{1} \sqcup \ldots \sqcup z_{n} \sqsubseteq z$ is equivalent to $z_{1} \sqsubseteq z \wedge \ldots \wedge z_{n} \sqsubseteq z$, it is easy to see that $\phi(\underline{x})$ is equivalent to a formula of the 
form

$$
\exists y .\left(\left(y \sqsubseteq t_{1}\right) \wedge \cdots \wedge\left(y \sqsubseteq t_{n}\right) \wedge\left(u_{1} \sqsubseteq s_{1} \sqcup y\right) \wedge \cdots \wedge\left(u_{m} \sqsubseteq s_{m} \sqcup y\right)\right),
$$

where $t_{i}, s_{j}, u_{k}$ are terms not involving $y$. Let $\phi^{*}(\underline{x})$ be the formula

$$
\bigwedge_{i=1}^{n} \bigwedge_{j=1}^{m}\left(u_{j} \sqsubseteq s_{j} \sqcup t_{i}\right),
$$

and let $T_{0}^{*}$ be obtained from $T_{0}$ by adding to it the universal closures of all formulae $\phi \leftrightarrow \phi^{*}$.

We prove that $T_{0}^{*}$ is contained in the theory of Boolean algebras. In fact, the system of disequations (4) is equivalent, in the theory of Boolean algebras, to

$$
\exists y .\left(\left(y \sqsubseteq t_{1}\right) \wedge \cdots \wedge\left(y \sqsubseteq t_{n}\right) \wedge\left(u_{1} \sqcap \neg s_{1} \sqsubseteq y\right) \wedge \cdots \wedge\left(u_{m} \sqcap \neg s_{m} \sqsubseteq y\right),\right.
$$

and hence to

$$
\left(u_{1} \sqcap \neg s_{1} \sqsubseteq t_{1} \sqcap \ldots \sqcap t_{n}\right) \wedge \cdots \wedge\left(u_{m} \sqcap \neg s_{m} \sqsubseteq t_{1} \sqcap \ldots \sqcap t_{n}\right) .
$$

Finally, it is easy to see that (7) and (5) are equivalent.

It is well-known that every semilattice embeds into a Boolean algebra. This can, for example, be shown as follows. Given a semilattice $\mathcal{S}=(S, \sqcup, \perp)$, just consider the Boolean algebra $\mathcal{B}=\left(2^{S}, \cap, S, \cup, \emptyset, \overline{(\cdot)}\right)$ given by the dual of the usual Boolean algebra formed by the powerset of $S$ : this means that as join in $\mathcal{B}$ we take the intersection of sets, as the least element $S$, as the meet the union of sets, as the greatest element $\emptyset$, and as the negation operation the set complement. It is easy to see that the map associating with $s \in S$ the set $\left\{s^{\prime} \mid s \sqsubseteq s^{\prime}\right\}$ is a semilattice embedding from $\mathcal{S}$ into $\mathcal{B}$.

This shows that $T_{0}^{*}$ is the positive-existential model completion of $T_{0}$. In addition, this implies that any Boolean-based theory $T$ is $T_{0}$-positive-existentially compatible since $T_{0}^{*}$ is contained in $T$. Consequently, Theorem 4.1 covers the case of a basic $\mathcal{E}$-connection, as introduced in Example 2.2 (see $\S 7$ for details).

EXAMPLE 4.7. Let us now turn to Example 2.3, i.e., to connections over the theory $T_{0}$ of distributive lattices with a least element $\perp$. This theory is obviously effectively locally finite, and it has a positive-existential model completion, which can be obtained as follows. Every term is equivalent modulo $T_{0}$ both to (i) a term that is a (possibly empty) finite join of (non-empty) finite meets of variables, and to (ii) a term that is a (non-empty) finite meet of (possibly empty) finite joins of variables. A simple existential formula with just one existential quantifier $\phi(\underline{x})$ is then easily seen to be equivalent to a formula of the form

$$
\exists y \cdot\left(\bigwedge_{i}\left(y \sqsubseteq u_{i}\right) \wedge \bigwedge_{j}\left(t_{j} \sqcap y \sqsubseteq z_{j}\right) \wedge \bigwedge_{k}\left(v_{k} \sqsubseteq y \sqcup w_{k}\right)\right),
$$

where $u_{i}, t_{j}, v_{k}, w_{k}$ are terms not involving $y$. Let $\phi^{*}(\underline{x})$ be the formula

$$
\bigwedge_{i, k}\left(v_{k} \sqsubseteq u_{i} \sqcup w_{k}\right) \wedge \bigwedge_{j, k}\left(v_{k} \sqcap t_{j} \sqsubseteq w_{k} \sqcup z_{j}\right),
$$

and let $T_{0}^{*}$ be obtained from $T_{0}$ by adding to it the universal closures of all formulae $\phi \leftrightarrow \phi^{*}$. 
We prove that $T_{0}^{*}$ is contained in the theory of Boolean algebras. In fact, the system of disequations (8) is equivalent, in the theory of Boolean algebras, to

$$
\exists y \cdot\left(\bigwedge_{i}\left(y \sqsubseteq u_{i}\right) \wedge \bigwedge_{j}\left(y \sqsubseteq \neg t_{j} \sqcup z_{j}\right) \wedge \bigwedge_{k}\left(v_{k} \sqcap \neg w_{k} \sqsubseteq y\right)\right),
$$

and hence to

$$
\bigwedge_{i, k}\left(v_{k} \sqcap \neg w_{k} \sqsubseteq u_{i}\right) \wedge \bigwedge_{j, k}\left(v_{k} \sqcap \neg w_{k} \sqsubseteq \neg t_{j} \sqcup z_{j}\right) .
$$

Finally, it is easy to see that (11) and (9) are equivalent.

Since every distributive lattice with least element embeds into a Boolean algebra, ${ }^{9}$ this shows that $T_{0}^{*}$ is the positive-existential model completion of $T_{0}$. In addition, this implies that any Boolean-based equational theory $T$ is $T_{0}$-positiveexistentially compatible since $T_{0}^{*}$ is contained in $T$. Consequently, Theorem 4.1 covers the case of a basic deterministic $\mathcal{E}$-connection, as introduced in Example 2.3 (see $\S 7$ details).

EXAMPLE 4.8. The previous example can be slightly varied, by considering the theory $T_{0}$ of bounded distributive lattices (i.e., distributive lattices with a least and a greatest element). Let us prove that its positive-existential model completion is the theory $T_{0}^{*}$ axiomatized by $T_{0}$ together with the (universal closure of the) formula

$$
\exists y \cdot((x \sqcap y \approx 0) \wedge(x \sqcup y \approx 1)) .
$$

Thus, $T_{0}^{*}$ is simply the theory of Boolean algebras, formulated in a complementfree signature. Since every bounded distributive lattice embeds into a Boolean algebra, and since the theory of Boolean algebras coincides with its own positiveexistential model completion because it is Gaussian (see Example 4.5), it is sufficient to show that every e-formula $\phi$ in the signature of Boolean algebras is equivalent to an e-formula in the complement-free subsignature. In fact, we can assume that $\phi$ is a conjunction of identities of the form

$$
1 \approx \neg x_{1} \sqcup \cdots \sqcup \neg x_{n} \sqcup y_{1} \sqcup \cdots \sqcup y_{m} ;
$$

these identities are in turn trivially equivalent to the inequations

$$
x_{1} \sqcap \cdots \sqcap x_{n} \sqsubseteq y_{1} \sqcup \cdots \sqcup y_{m},
$$

which can obviously be transformed into identities between term in the complementfree subsignature.

Again this implies that every Boolean-based equational theory is $T_{0}$-compatible and that Theorem 4.1 covers the case of a basic functional $\mathcal{E}$-connection, as introduced in Example 2.3 (see again $\S 7$ for details).

EXAMPLE 4.9. Here we give an example with a non-functional signature. Let $T_{0}$ be the (obviously locally finite) theory of partial orders (posets). The positiveexistential model completion $T_{0}^{*}$ of $T_{0}$ is the theory axiomatized by $T_{0}$ together

\footnotetext{
${ }^{9}$ It is well-known that distributive lattices with least and greatest elements embed into Boolean algebras, and it is easy to embed a distributive lattice with least element into one with least and greatest elements by just adding a greatest element.
} 
with the axioms

$$
\exists x .\left(\bigwedge_{i}\left(x \sqsubseteq a_{i}\right) \wedge \bigwedge_{j}\left(b_{j} \sqsubseteq x\right)\right) \leftrightarrow \bigwedge_{i, j}\left(b_{j} \sqsubseteq a_{i}\right),
$$

where $i, j$ range over a finite index set and $a_{i}, b_{j}$ are variables.

To embed a model $(P, \sqsubseteq)$ of $T_{0}$ into a model of $T_{0}^{*}$, just take the poset of downward closet subsets of $(P, \sqsubseteq)$. A downward closed subset of $P$ is a set $X \subseteq P$ such that $x \in X$ and $y \sqsubseteq x$ imply $y \in X$. These sets are ordered by set inclusion. It is easy to see that this yields a model of $T_{0}^{*}$. In fact, it is enough to show that, given downward closed sets $A_{i}, B_{j}$ satisfying $\bigwedge_{i, j}\left(B_{j} \sqsubseteq A_{i}\right)$, there is a downward closed set $X$ such that $\bigwedge_{i}\left(X \sqsubseteq A_{i}\right) \wedge \bigwedge_{j}\left(B_{j} \sqsubseteq X\right)$. Since the union of downward closed sets is again downward closed, we can take the union of the $B_{j}$ as the set $X$. The embedding of $(P, \sqsubseteq)$ into downward closed sets is obtained by associating with $a \in P$ the cone $a \downarrow:=\{b \mid b \sqsubseteq a\}$. It is easy to see that $a \sqsubseteq a^{\prime}$ iff $a \downarrow \subseteq a^{\prime} \downarrow$.

In order to obtain a $T_{0}$-positive-existentially compatible theory, we consider again the theory $T$ of semilattices, but now we assume that the symbol $\sqsubseteq$ belongs to the signature, and satisfies the axiom $x \sqsubseteq y \leftrightarrow x \wedge y \approx y$. The theory $T$ is $T_{0}$-positive-existentially compatible since every model of $T$ is a model of $T_{0}^{*}$ : in fact

$$
\exists x .\left(\bigwedge_{i}\left(x \sqsubseteq a_{i}\right) \wedge \bigwedge_{j}\left(b_{j} \sqsubseteq x\right)\right)
$$

is equivalent (in the theory $T$ ) to

$$
\exists x .\left(\bigwedge_{i}\left(x \sqsubseteq a_{i}\right) \wedge\left(\bigsqcup_{j} b_{j} \sqsubseteq x\right)\right),
$$

i.e., to

$$
\bigwedge_{i}\left(\bigsqcup_{j} b_{j} \sqsubseteq a_{i}\right)
$$

and thus to $\bigwedge_{i, j}\left(b_{j} \sqsubseteq a_{i}\right)$.

Other theories that extend $T_{0}^{*}$ (and are hence $T_{0}$-positive-existentially compatible) are theories that extend the theory of total orders, as is easily seen.

EXAMPLE 4.10. Here we go back to the theories introduced in the proof of Theorem 2.5 (which gives an example of theories $T_{0}, T_{1}, T_{2}$ for which decidability of the universal fragment does not transfer to their connection), and analyze which of our conditions are violated by them. Let $T_{0}^{\prime}$ be the one-sorted theory of pure equality and let $\left.T_{0}:=T_{0}^{\prime}\right\urcorner$ be as in the proof of Theorem 2.5. It is easily seen that $T_{0}$ has a positive-existential model completion $T_{0}^{*}$, which is axiomatized by using infinitely many sentences jointly saying that the domain is infinite. It follows that a (one-sorted) theory $T \supseteq T_{0}$ is $T_{0}$-positive-existentially compatible iff every model of $T$ embeds into an infinite model of $T$. This condition (in an equivalent formulation) is known as stable infiniteness: it is the standard NelsonOppen combinability requirement [23, 22] for disjoint signatures. Most theories considered in Computer Science applications are stably infinite. However, notice that, in a stably infinite theory, there is no difference between satisfiability of a constraint in a model of $T$ and satisfiability in an infinite model of $T$. Thus, theories satisfying the statement of Lemma 2.4 cannot be stably infinite. This 
shows that it is precisely the $T_{0}$-positive-existential compatibility requirement for $T_{2}$ that is violated in the example provided in the proof of Theorem 2.5.

§5. A variant of the connection scheme. Here we consider a slightly different combination scheme where a theory $T$ is connected with itself w.r.t. a subtheory $T_{0}$. Let $T_{0} \subseteq T$ be theories over the respective signatures $\Omega_{0} \subseteq \Omega$. We use $T_{>T_{0}}$ to denote the theory whose models are models $\mathcal{M}$ of $T$ endowed with a homomorphism $h: \mathcal{M}_{\mid \Omega_{0}} \rightarrow \mathcal{M}_{\mid \Omega_{0}}$. Thus, the signature $\Omega^{\prime}$ of $T_{>T_{0}}$ is obtained from the signature $\Omega$ of $T$ by adding a new function symbol $h_{S}$ of arity $S S$ for every sort $S$ of $\Omega_{0}$. The axioms of $T_{>T_{0}}$ are obtained from the axioms of $T$ by adding

$$
\begin{aligned}
& h_{S}\left(f\left(x_{1}, \ldots, x_{n}\right)\right) \approx f\left(h_{S_{1}}\left(x_{1}\right), \ldots, h_{S_{n}}\left(x_{n}\right)\right), \\
& P\left(x_{1}, \ldots, x_{n}\right) \rightarrow P\left(h_{S_{1}}\left(x_{1}\right), \ldots, h_{S_{n}}\left(x_{n}\right)\right),
\end{aligned}
$$

for every function (predicate) symbol $f(P)$ in $\Omega_{0}$ of arity $S_{1} \ldots S_{n} S\left(S_{1} \ldots S_{n}\right)$.

EXAMPLE 5.1. An interesting example of a theory obtained as such a connection is the theory $E_{\mathbf{K}}$ corresponding to the basic modal logic $\mathbf{K}$. In fact, let $T$ be the theory of Boolean algebras, and $T_{0}$ the theory of semilattices over the signature $\Omega_{0}$ as defined in Example 2.2. If we use the symbol $\diamond$ for the connection function, then $T_{>T_{0}}$ is exactly the theory $E_{\mathbf{K}}$.

5.1. A non-deterministic combination procedure. In this subsection we state the main decidability transfer result. The approach is analogous to the one chosen in $\S 4$, and it leads to a non-deterministic combination procedure. In the next subsection we show that, under certain additional restrictions, this non-deterministic procedure can be replaced by a deterministic one.

THEOREM 5.2. Let $T_{0}, T$ be theories over the respective signatures $\Omega_{0}, \Omega$, where $\Omega_{0}$ is a subsignature of $\Omega$. Assume that $T_{0} \subseteq T$, that $T_{0}$ is universal and locally finite, and that $T$ is $T_{0}$-positive-existentially compatible. Then the decidability of the universal fragment of $T$ entails the decidability of the universal fragment of $T_{>T_{0}}$.

To prove the theorem, we consider a finite set $\Gamma \cup \Gamma_{0}$ of ground flat literals over the signature $\Omega^{\prime}$ of $T_{>T_{0}}$, where $\Gamma$ is a set of literals in the signature $\Omega$ of $T$ (expanded with free constants), and $\Gamma_{0}$ is of the form

$$
\Gamma_{0}=\left\{h\left(a_{1}\right) \approx b_{1}, \ldots, h\left(a_{n}\right) \approx b_{n}\right\} .
$$

The theorem is an easy consequence of the following proposition, whose proof is similar to the one of Proposition 4.2.

Proposition 5.3. The constraint $\Gamma \cup \Gamma_{0}$ is satisfiable in $T_{>T_{0}}$ iff there exists a triple $(\mathcal{A}, \mathcal{B}, \nu)$ such that

1. $\mathcal{A}$ is an $\Omega_{0}$-model of $T_{0}$, which is generated by $\left\{a_{1}^{\mathcal{A}}, \ldots, a_{n}^{\mathcal{A}}\right\} ;$

2. $\mathcal{B}$ is an $\Omega_{0}$-model of $T_{0}$, which is generated by $\left\{b_{1}^{\mathcal{B}}, \ldots, b_{n}^{\mathcal{B}}\right\}$;

3. $\nu: \mathcal{A} \rightarrow \mathcal{B}$ is an $\Omega_{0}$-homomorphism such that $\nu\left(a_{j}^{\mathcal{A}}\right)=b_{j}^{\mathcal{B}}$ for $j=1, \ldots, n$;

4. $\Gamma \cup \Delta_{\Omega_{0}}(\mathcal{A}) \cup \Delta_{\Omega_{0}}(\mathcal{B})$ is satisfiable in $T$. 
Proof. The only-if direction is again simple. To prove the if direction, assume that there is a triple $(\mathcal{A}, \mathcal{B}, \nu)$ satisfying $1 .-4$. of the proposition. In particular, this means that $\Gamma \cup \Delta_{\Omega_{0}}(\mathcal{A}) \cup \Delta_{\Omega_{0}}(\mathcal{B})$ is satisfiable in a model $\mathcal{N}$ of $T$. We can assume without loss of generality that $\mathcal{N}$ is an at most countable model of $T \cup T_{0}^{*}$. By Robinson's diagram theorem, $\mathcal{A}, \mathcal{B}$ are $\Omega_{0}$-substructures of $\mathcal{N}$. Using the same argument as in the proof of Proposition 4.2, we can extend the $\Omega_{0}$-homomorphism $\nu: \mathcal{A} \rightarrow \mathcal{B}$ to an $\Omega_{0}$-endomorphism $\nu_{\infty}: \mathcal{N}_{\mid \Omega_{0}} \rightarrow \mathcal{N}_{\mid \Omega_{0}}$. The pair $\left(\mathcal{N}, \nu_{\infty}\right)$ yields a model of $T_{>T_{0}}$ that satisfies $\Gamma \cup \Gamma_{0}$.

Obviously, this proposition gives rise to a non-deterministic decision procedure for the universal fragment of $T_{>T_{0}}$, which is analogous to the one described in the proof of Theorem 4.1

Applied to the connection of the theory $B A$ of Boolean algebras with itself w.r.t. the theory of semilattices considered in Example 5.1, the proof of Theorem 5.2 shows that deciding the universal theory of $E_{\mathbf{K}}$ can be reduced to deciding the universal theory of $B A$. It is well-known that deciding the universal theory of $E_{\mathbf{K}}$ is equivalent to deciding global consequence in $\mathbf{K}$, and that deciding the universal theory of $B A$ is equivalent to propositional reasoning. Thus, we have shown the (rather surprising) result that the global consequence problem in $\mathbf{K}$ can be reduced to purely propositional reasoning. However, if we directly apply the non-deterministic combination algorithm suggested by Proposition 5.3, then the complexity of the obtained decision procedure is worse then the known ExpTime-complexity [29] of the problem. The deterministic combination procedure described below overcomes this problem.

5.2. A deterministic combination procedure. As pointed out in [25], Nelson-Oppen style combination procedures can be made deterministic in the presence of a certain convexity condition. Let $T$ be a theory over the signature $\Omega$, and let $\Omega_{0}$ be a subsignature of $\Omega$. Following [30], we say that $T$ is $\Omega_{0}$ convex iff every finite set of ground $\Omega^{X}$-literals (using additional free constants from $X$ ) $T$-entailing a disjunction of $n>1 \Omega_{0}^{X}$-atoms, already $T$-entails one of the disjuncts. Note that universal Horn $\Omega$-theories are always $\Omega$-convex. In particular, this means that equational theories (like $B A$ ) are convex w.r.t. any subsignature.

Let $T_{0} \subseteq T$ be theories over the respective signatures $\Omega_{0}, \Omega$, where $\Omega_{0}$ is a subsignature of $\Omega$. If $T$ is $\Omega_{0}$-convex, then Theorem 5.2 can be shown with the help of a deterministic combination procedure. (The same is actually also true for Theorem 4.1 and Theorem 4.3, but this will not explicitly be shown here.)

Let $\Gamma \cup \Gamma_{0}$ be a finite set of ground literals (with free constants) in the signature of $T_{>T_{0}}$; suppose also that $\Gamma$ does not contain the symbol $h$ and that $\Gamma_{0}=$ $\left\{h\left(a_{1}\right) \approx b_{1}, \ldots, h\left(a_{n}\right) \approx b_{n}\right\}$. We say that $\Gamma$ is $\Gamma_{0}$-saturated iff for every $\Omega_{0^{-}}$ atom $\alpha\left(x_{1}, \ldots, x_{n}\right), T \cup \Gamma \models \alpha\left(a_{1}, \ldots, a_{n}\right)$ implies $\alpha\left(b_{1}, \ldots, b_{n}\right) \in \Gamma$.

THEOREM 5.4. Let $T_{0}, T$ be theories over the respective signatures $\Omega_{0}, \Omega$, where $\Omega_{0}$ is a subsignature of $\Omega$. Assume that $T_{0} \subseteq T$, that $T_{0}$ is universal and locally finite, and that $T$ is $\Omega_{0}$-convex and $T_{0}$-positive-existentially compatible. Then the following deterministic procedure decides whether $\Gamma \cup \Gamma_{0}$ is satisfiable in $T_{>T_{0}}$ (where $\Gamma, \Gamma_{0}$ are as in Proposition 5.3):

1. $\Gamma_{0}$-saturate $\Gamma$; 
2. check whether the $\Gamma_{0}$-saturated set $\widehat{\Gamma}$ obtained this way is satisfiable in $T$.

Proof. The saturation process (and thus the procedure) terminates because $T_{0}$ is locally finite (it should be clear that saturation is done modulo $T_{0}$ ). In addition, if $\Gamma \cup \Gamma_{0}$ is satisfied in a model $\mathcal{M}$ of $T_{>T_{0}}$, then the reduct of $\mathcal{M}$ to the signature $\Omega$ obviously satisfies $\widehat{\Gamma}$.

Conversely, if the $\Gamma_{0}$-saturated set $\widehat{\Gamma}$ is satisfiable in $T$, then we use $\widehat{\Gamma}$ to construct a triple $(\mathcal{A}, \mathcal{B}, \nu)$ satisfying 1.-4 of Proposition 5.3. Since $\widehat{\Gamma}$ is satisfiable in $T$, and $T$ is $\Omega_{0}$-convex, the following two finite ${ }^{10}$ sets of literals are both satisfiable in $T_{0}$ (where $\underline{a}$ abbreviate $a_{1}, \ldots, a_{n}$ and let $\underline{b}$ abbreviate $b_{1}, \ldots, b_{n}$ ):

$$
\begin{array}{rlll}
\Gamma_{\underline{a}}:= & \{\alpha(\underline{a}) \mid T \cup \widehat{\Gamma} \models \alpha(\underline{a})\} & \cup & \{\neg \alpha(\underline{a})|T \cup \widehat{\Gamma} \not| \alpha(\underline{a})\}, \\
\Gamma_{\underline{b}}:= & \{\alpha(\underline{b}) \mid T \cup \widehat{\Gamma} \models \alpha(\underline{b})\} & \cup & \{\neg \alpha(\underline{b}) \mid T \cup \widehat{\Gamma} \not \models \alpha(\underline{b})\},
\end{array}
$$

where $\alpha(\underline{x})$ ranges over $\Omega_{0}$-atoms (modulo $T_{0}$ ). In fact, assume (without loss of generality) that $\Gamma_{\underline{a}}$ is not satisfiable in $T_{0}$. This means that

$$
T_{0} \cup\{\alpha(\underline{a}) \mid T \cup \widehat{\Gamma} \models \alpha(\underline{a})\} \mid=\bigvee_{T \cup \widehat{\Gamma} \not<\alpha(\underline{a})} \alpha(\underline{a}),
$$

Since $T_{0} \subseteq T$ and $T$ is $\Omega_{0}$-convex, this implies that $T \cup\{\alpha(\underline{a})|T \cup \widehat{\Gamma}|=\alpha(\underline{a})\} \models$ $\alpha^{\prime}(\underline{a})$ for some $\Omega_{0}$-atom $\alpha^{\prime}(\underline{x})$ such that $T \cup \widehat{\Gamma} \not \forall \alpha^{\prime}(\underline{a})$. However, $T \cup\{\alpha(\underline{a}) \mid$ $T \cup \widehat{\Gamma} \models \alpha(\underline{a})\} \models \alpha^{\prime}(\underline{a})$ obviously implies $T \cup \widehat{\Gamma} \models \alpha^{\prime}(\underline{a})$, which yields the desired contradiction.

Pick a pair of models of $T_{0}$ satisfying $\Gamma_{\underline{a}}$ and $\Gamma_{\underline{b}}$, and let $\mathcal{A}, \mathcal{B}$ be their $\Omega_{0^{-}}$ substructures generated by (the interpretations of) $\underline{a}$ and $\underline{b}$, respectively. Since $T_{0}$ is universal, $\mathcal{A}$ and $\mathcal{B}$ are models of $T_{0}$. Moreover, by construction, for every $\Omega_{0^{-}}$ atom $\alpha(\underline{x})$ we have that $T \cup \widehat{\Gamma} \models \alpha(\underline{a})$ iff $\mathcal{A} \models \alpha(\underline{a})$ and, similarly, $T \cup \widehat{\Gamma} \models \alpha(\underline{b})$ iff $\mathcal{B}=\alpha(\underline{b})$. As a consequence, the $\Gamma_{0}$-saturatedness of $\widehat{\Gamma}$ and Robinson's diagram theorem guarantee that the map associating $b_{i}$ with $a_{i}$ can be extended to a homomorphism $\nu: \mathcal{A} \rightarrow \mathcal{B}$.

It remains to show that $\widehat{\Gamma} \cup \Delta_{\Omega_{0}}(\mathcal{A}) \cup \Delta_{\Omega_{0}}(\mathcal{B})$ is satisfiable in $T$ (since $\Gamma \subseteq \widehat{\Gamma}$, this implies that $\Gamma \cup \Delta_{\Omega_{0}}(\mathcal{A}) \cup \Delta_{\Omega_{0}}(\mathcal{B})$ is satisfiable in $T$ ). Taking into consideration the $\Omega_{0}$-convexity of $T$ and the fact that $\widehat{\Gamma}$ is satisfiable in $T$, satisfiability of $\widehat{\Gamma} \cup \Delta_{\Omega_{0}}(\mathcal{A}) \cup \Delta_{\Omega_{0}}(\mathcal{B})$ in $T$ means that for no atom $\alpha(\underline{a})$ false in $\mathcal{A}(\alpha(\underline{b})$ false in $\mathcal{B})$ we have that $T \cup \widehat{\Gamma} \cup \Delta_{\Omega_{0}}^{+}(\mathcal{A}) \cup \Delta_{\Omega_{0}}^{+}(\mathcal{B})=\alpha(\underline{a})\left(T \cup \widehat{\Gamma} \cup \Delta_{\Omega_{0}}^{+}(\mathcal{A}) \cup \Delta_{\Omega_{0}}^{+}(\mathcal{B}) \models \alpha(\underline{b})\right) .{ }^{11}$ However, as remarked above, $T \cup \widehat{\Gamma} \models \alpha(\underline{a})$ holds iff $\mathcal{A} \models \alpha(\underline{a})$ holds (and similarly for $\mathcal{B})$. This means that $T \cup \widehat{\Gamma} \cup \Delta_{\Omega_{0}}^{+}(\mathcal{A}) \cup \Delta_{\Omega_{0}}^{+}(\mathcal{B})$ is the same theory as $T \cup \widehat{\Gamma}$. But then the claim that "for no atom $\alpha(\underline{a})$ false in $\mathcal{A}$ (or $\alpha(\underline{b})$ false in $\mathcal{B})$ we have that $T \cup \widehat{\Gamma} \models \alpha(\underline{a})(T \cup \widehat{\Gamma} \models \alpha(\underline{b}))$ " becomes trivial, once again because $T \cup \widehat{\Gamma} \models \alpha(\underline{a})$ is equivalent to $\mathcal{A} \models \alpha(\underline{a})(T \cup \widehat{\Gamma} \models \alpha(\underline{b})$ is equivalent to $\mathcal{B} \mid=\alpha(\underline{b}))$.

\footnotetext{
${ }^{10}$ It goes without saying that "finiteness" here means "finiteness modulo $T_{0}$;" see the definition of local finiteness.

${ }^{11}$ Recall that $\Delta_{\Omega_{0}}^{+}(\mathcal{A})$ denotes the positive diagram of $\mathcal{A}$, i.e., it consists of those atoms true in $\mathcal{A}$. Also note that $\neg \alpha(\underline{a}) \in \Delta_{\Omega_{0}}(\mathcal{A}) \backslash \Delta_{\Omega_{0}}^{+}(\mathcal{A})$ iff the atom $\alpha(\underline{a})$ is false in $\mathcal{A}$.
} 
Example 5.1 (continued) Let us come back to the connection of $T:=B A$ with itself w.r.t. the theory $T_{0}$ of semilattices, which yields as combined theory the equational theory $E_{\mathbf{K}}$ corresponding to the basic modal logic $\mathbf{K}$. In this case, checking during the saturation process whether $T \cup \Gamma \models \alpha(\underline{a})$ amounts to checking whether a propositional formula $\phi_{\Gamma}$ (whose size is linear in the size of $\Gamma$ ) implies a propositional formula of the form $\psi_{1} \Leftrightarrow \psi_{2}$, where $\psi_{1}, \psi_{2}$ are disjunctions of the propositional variables from $a$. Since propositional reasoning can be done in time exponential in the number of propositional variables, and there are only exponentially many different formulae of the form $\psi_{1} \Leftrightarrow \psi_{2}$, the saturation process needs at most exponential time. The size of the $\Gamma_{0}$-saturated set $\widehat{\Gamma}$ may be exponential in the size of $\Gamma$, but it still contains only the free constants $\underline{a}$. Consequently, testing satisfiability of $\widehat{\Gamma}$ in $T$ is again a propositional reasoning problem that can be done in time exponential in the number of free constants $\underline{a}$.

Consequently, we have shown that Theorem 5.4 yields an ExpTime decision procedure for the global consequence relation in $\mathbf{K}$, which thus matches the known worst-case complexity of the problem.

§6. Conditions on the connection functions. Until now, we have considered connection functions that are arbitrary homomorphisms. In this section we impose the additional conditions that the connection functions be surjective, embeddings, or isomorphisms: in this way, we obtain new combined theories, which we denote by $T_{1}>{ }_{T_{0}}^{e m} T_{2}, T_{1}>_{T_{0}}^{s} T_{2}, T_{1}>_{T_{0}}^{i s o} T_{2}$, respectively. This defines the combined theories in a model-theoretic way. One can also give an axiomatic description of $T_{1}>{ }_{T_{0}}^{e m} T_{2}, T_{1}>_{T_{0}}^{s} T_{2}$, and $T_{1}>_{T_{0}}^{i s o} T_{2}$. For example, the axioms of $T_{1}>_{T_{0}}^{s} T_{2}$ are obtained from the ones of $T_{1}>_{T_{0}} T_{2}$ by adding axioms expressing that $h$ is surjective, i.e., for every sort $S$ in $\Omega_{0}$ we add the axiom

$$
\forall y \cdot \exists x \cdot h_{S}(x)=y,
$$

where $x$ is a variable of sort $S^{1}$ and $y$ a variable of sort $S^{2}$.

For these combined theories one can show combination results that are analogous to Theorem 4.1: one just needs different compatibility conditions. To treat embeddings and isomorphisms, we use the compatibility condition introduced in $[16,4,5]$ for the case of unions of theories. Following $[16,4,5]$, we call this condition $T_{0}$-compatibility in the following.

In order to define this notion of compatibility, we need to introduce the notion of a model completion. The definition given below differs from the one given in $[16,4,5]$. However, the two notions can be shown to be equivalent for universal theories (see Proposition 8.4 in $\$ 8$ ), and we will apply the definition only to theories that are universal. The reason for giving an alternative formulation is that it makes the connection between a model completion and a positiveexistential model completion more transparent.

DeFinition 6.1. Let $T$ be a universal $\Omega$-theory and let $T^{*}$ be an $\Omega$-theory. We say that $T^{*}$ is a model completion of $T$ iff the following conditions are satisfied:

1. $T \subseteq T^{*}$;

2. every model of $T$ embeds into a model of $T^{*}$; 
3. for every formula $\phi(\underline{x})$ there is an open formula $\phi^{*}(\underline{x})$ such that

$$
T^{*} \models \phi \leftrightarrow \phi^{*} .
$$

It can be shown that the models of $T^{*}$ are just the existentially closed models of $T$ (see [11] or $\S 8)$.

Definition 6.2. Let $T_{0} \subseteq T$ be theories over the respective signatures $\Omega_{0} \subseteq \Omega$. We say that $T$ is $T_{0}$-compatible iff $T_{0}$ is universal, has a model completion $T_{0}^{*}$, and every model of $T$ embeds into a model of $T \cup T_{0}^{*}$.

The notions of a model completion and of $T_{0}$-compatibility can actually be viewed as special cases of the notions of a positive-existential model completion and of $T_{0}$-positive-existential compatibility. To show this, we employ a notation introduced in Section 2.3. Recall that, given a theory $T$ over the signature $\Omega$, the theory $T\urcorner$ over the signature $\Omega\urcorner$ is defined as follows: for every $n$-ary predicate symbol $P$ of $\Omega$, the signature $\Omega\urcorner$ extends $\Omega$ by the additional new $n$-ary predicate symbol $P\urcorner$, and the theory $T\urcorner$ contains in addition to the axioms of $T$ the axioms

$$
\left.\neg P\left(x_{1}, \ldots, x_{n}\right) \leftrightarrow P\right\urcorner\left(x_{1}, \ldots, x_{n}\right) .
$$

The following proposition states some relevant facts about the connection between $T$ and $T\urcorner$ :

Proposition 6.3. Let $T$ be a universal $\Omega$-theory, and $T_{0} \subseteq T$ a theory over the subsignature $\Omega_{0}$ of $\Omega$.

1. The theory $T\urcorner$ is a universal $\Omega\urcorner$-theory.

2. The universal fragment of $T$ is decidable iff the universal fragment of $T\urcorner$ is decidable.

3. $T\urcorner$ has a positive-existential model completion iff $T$ has a model completion.

4. $T\urcorner$ is $T_{0}^{\urcorner}$-positive-existentially compatible iff $T$ is $T_{0}$-compatible.

Proof. The first two facts follow immediately from our definitions.

To prove the third fact, first assume that $T\urcorner$ has a positive-existential model completion $T^{\neg *}$. It is easy to see that the theory $T^{*}$ obtained from $T^{\neg *}$ by replacing all predicates $P \neg$ by $\neg P$ is a model completion of $T$. To see this, note that one can eliminate quantifier from arbitrary formulae as soon as one can eliminate quantifiers from existentially quantified conjunctions of literals (see [11], Lemma 1.5.1). In addition, atoms in $T\urcorner$ correspond to literals in $T$.

Conversely, assume that $T$ has a model completion $T^{*}$. It would be tempting to claim that $\left.T^{*}\right\urcorner$ is a positive-existential model completion of $\left.T\right\urcorner$. However, recall that we require the positive-existential model completion of $T\urcorner$ to be a geometric theory. This need not be the case for $\left.T^{*}\right\urcorner$ since the model completion $T^{*}$ of $T$ need not be a geometric theory. In order to define the positive-existential model completion of $T\urcorner$, we recall from Section 4.3 that it is enough to (i) produce for every simple existential formula $\phi(\underline{x})$ an open and geometric formula $\phi^{*}(\underline{x})$; (ii) extend $T^{\urcorner}$to $T^{\neg *}$ by adding the formulae $\phi(\underline{x}) \leftrightarrow \phi^{*}(\underline{x})$ for every simple existential formula $\phi(\underline{x})$; and (iii) show that every model of $T\urcorner$ embeds into a model of $T^{\neg *}$.

Given a simple existential formula $\phi(\underline{x})$ in the signature $\Omega\urcorner$ of $T\urcorner$, we can first replace all predicates $P^{\urcorner}$in $\phi(\underline{x})$ by $\neg P$, which yields a formula $\phi^{\prime}(\underline{x})$ in the 
signature $\Omega$ of $T$. Since $T^{*}$ is a model completion of $T$, we know that there is an open formula $\phi^{\prime *}(\underline{x})$ over the signature $\Omega$ such that $T^{*} \models \phi^{\prime}(\underline{x}) \leftrightarrow \phi^{\prime *}(\underline{x})$. Let $\phi^{*}(\underline{x})$ be the formula obtained from $\phi^{\prime *}(\underline{x})$ by replacing all negated predicates $\neg P$ by $P^{\urcorner}$, and let $T^{\neg *}$ be the theory obtained from $\left.T\right\urcorner$ by adding the formulae $\phi(\underline{x}) \leftrightarrow \phi^{*}(\underline{x})$ for every simple existential formula $\phi(\underline{x})$.

To show that every model of $T\urcorner$ embeds into a model of $T^{\neg *}$, let $\left.\mathcal{A}\right\urcorner$ be a model of $T\urcorner$. Then the reduct $\mathcal{A}$ of $\mathcal{A}\urcorner$ to $\Omega$ can be embedded into a model $\mathcal{B}$ of $T^{*}$. Let $\left.\mathcal{B}\right\urcorner$ be the expansion of $\mathcal{B}$ to $\left.\Omega\right\urcorner$ that interprets the predicates $\left.P\right\urcorner$ by the complements of the interpretations of the predicates $P$. Then $\mathcal{B}\urcorner$ is a model of $T\urcorner$, and obviously $\mathcal{A}\urcorner$ embeds into $\mathcal{B}\urcorner$. In addition, for every simple existential formula $\phi(\underline{x})$, the facts that $T^{*} \models \phi^{\prime}(\underline{x}) \leftrightarrow \phi^{\prime *}(\underline{x})$, that $\mathcal{B}$ is a model of $T^{*}$, and that $\left.\mathcal{B}\right\urcorner$ is a model of $\left.T\right\urcorner$ imply that $\left.\mathcal{B}\right\urcorner$ is a model of $\phi(\underline{x}) \leftrightarrow \phi^{*}(\underline{x})$. This shows that $\mathcal{A}\urcorner$ embeds into the model $\mathcal{B}\urcorner$ of $T^{\sim *}$.

Finally, the fourth fact is an easy consequence of the third. Basically, the only additional things to prove are the embedding conditions. One direction is again simple, and the other can be shown similarly to the proof of the embedding condition above.

6.1. Embeddings as connection functions. Let us first investigate the case of connection functions that are embeddings.

THEOREM 6.4. Let $T_{0}, T_{1}$, and $T_{2}$ be theories over the respective signatures $\Omega_{0}, \Omega_{1}$, and $\Omega_{2}$, where $\Omega_{0}$ is a common subsignature of $\Omega_{1}$ and $\Omega_{2}$. Assume that $T_{0} \subseteq T_{1}$ and $T_{0} \subseteq T_{2}$, and that $T_{0}$ is universal and locally finite. If $T_{2}$ is $T_{0}$-compatible, then the decidability the universal fragments of $T_{1}$ and $T_{2}$ entails the decidability of the universal fragment of $T_{1}>_{T_{0}}^{e m} T_{2}$.

Proof. This theorem is an easy consequence of Theorem 4.1 and Proposition 6.3. In fact, by Proposition 6.3, the preconditions of the theorem imply that the theories $T_{0}^{\urcorner}, T_{1}, T_{2}^{\urcorner}$satisfy the preconditions of Theorem 4.1. Thus, we know that the universal theory of $T_{1}^{\neg}>_{T_{0}} T_{2}^{\urcorner}$is decidable.

Let $\Omega$ be the signature of $T_{1}>_{T_{0}}^{e m} T_{2}$, and $\left.\Omega\right\urcorner$ the signature of $T_{1}^{\neg}>_{T_{0}} T_{2}^{\urcorner}$. To show decidability of the universal fragment of $T_{1}>{ }_{T_{0}}^{e m} T_{2}$, it is sufficient to show that an $\Omega$-constraint is satisfiable in a model of $T_{1}^{\neg}>_{T_{0}} T_{2}^{\urcorner}$iff it is satisfiable in a model of $T_{1}>_{T_{0}}^{e m} T_{2}$. However, the models of $T_{1}^{\neg}>_{T_{0}} T_{2}^{\urcorner}$are of the form $\left(\mathcal{M}^{1}, \mathcal{M}^{2}, g\right)$, where $\mathcal{M}^{1}$ is a model of $T_{1}^{\neg}, \mathcal{M}^{2}$ is a model of $T_{2}^{\neg}$, and $g$ is an $\Omega_{0}^{\neg}$-homomorphism. The reducts $\mathcal{N}^{1}, \mathcal{N}^{2}$ of $\mathcal{M}^{1}, \mathcal{M}^{2}$ to the signatures $\Omega_{1}, \Omega_{2}$ are models of $T_{1}, T_{2}$, respectively, and the reduct $h$ of $g$ to $\Omega_{0}$ is an $\Omega_{0}$-embedding. Thus, $\left(\mathcal{N}^{1}, \mathcal{N}^{2}, h\right)$ is a model of $T_{1}>{ }_{T_{0}}^{e m} T_{2}$, which obviously satisfies the same $\Omega$ constraints as $\left(\mathcal{M}^{1}, \mathcal{M}^{2}, g\right)$. Conversely, every model of $T_{1}>_{T_{0}}^{e m} T_{2}$ can uniquely by expanded to a model of $T_{1}^{\urcorner}>_{T_{0}} T_{2}^{\urcorner}$that satisfies the same $\Omega$-constraints by defining the interpretations of the predicates $P\urcorner$ as the complement of the interpretations of the predicates $P$.

Of course, Theorem 6.4 can also be proved directly by using an approach analogous to the one employed in the proof of Theorem 4.1. Assume that the constraint $\Gamma$ consists of ground flat literals over the signature $\Omega$ of $T_{1}>_{T_{0}}^{e m} T_{2}$ (with additional free constants). Since all literals in $\Gamma$ are flat, we can divide $\Gamma$ into three disjoint sets $\Gamma=\Gamma_{0} \cup \Gamma_{1} \cup \Gamma_{2}$ where $\Gamma_{i}(i=1,2)$ is a set of literals in 
the signature $\Omega_{i}$ (expanded with free constants), and $\Gamma_{0}$ is of the form

$$
\Gamma_{0}=\left\{h\left(a_{1}\right) \approx b_{1}, \ldots, h\left(a_{n}\right) \approx b_{n}\right\}
$$

for free constants $a_{1}, b_{1}, \ldots, a_{n}, b_{n}$. Theorem 6.4 easily follows from the next proposition, which in turn is an easy consequence of Proposition 4.2 and Proposition 6.3.

Proposition 6.5. The constraint $\Gamma=\Gamma_{0} \cup \Gamma_{1} \cup \Gamma_{2}$ is satisfiable in $T_{1}>{ }_{T_{0}}^{e m} T_{2}$ iff there exists a triple $(\mathcal{A}, \mathcal{B}, \nu)$ such that

1. $\mathcal{A}$ is an $\Omega_{0}$-model of $T_{0}$, which is generated by $\left\{a_{1}^{\mathcal{A}}, \ldots, a_{n}^{\mathcal{A}}\right\}$;

2. $\mathcal{B}$ is an $\Omega_{0}$-model of $T_{0}$, which is generated by $\left\{b_{1}^{\mathcal{B}}, \ldots, b_{n}^{\mathcal{B}}\right\}$;

3. $\nu: \mathcal{A} \rightarrow \mathcal{B}$ is an $\Omega_{0}$-embedding such that $\nu\left(a_{j}^{\mathcal{A}}\right)=b_{j}^{\mathcal{B}}$ for $j=1, \ldots, n$;

4. $\Gamma_{1} \cup \Delta_{\Omega_{0}}(\mathcal{A})$ is satisfiable in $T_{1}$;

5. $\Gamma_{2} \cup \Delta_{\Omega_{0}}(\mathcal{B})$ is satisfiable in $T_{2}$.

6.2. Surjective connections. To treat $T_{1}>_{T_{0}}^{s} T_{2}$, we must dualize the notions "positive-existential model completion" and "positive-existential compatibility". These notions are based on co-geometric formulae, which are the dual of geometric formulae in the sense that existential quantification is replaced by universal quantification. A co-geometric formula is a formula built from atoms by using conjunction, disjunction and universal quantification. Similarly, a cogeometric theory is a theory axiomatized by (universal closure of) implications of co-geometric formulae.

Definition 6.6. Let $T$ be a universal $\Omega$-theory, and let $T^{*}$ be an $\Omega$-theory. We say that $T^{*}$ is a positive-universal model completion of $T$ iff the following conditions are satisfied:

1. $T \subseteq T^{*}$;

2. every model of $T$ embeds into a model of $T^{*}$;

3. for every co-geometric formula $\phi(\underline{x})$ there is an open co-geometric formula $\phi^{*}(\underline{x})$ such that

$$
T^{*} \models \phi \leftrightarrow \phi^{*} .
$$

The new notion of compatibility defined below differs from the one introduced in $\S 3$ in that positive-existential model completions are replaced by positive-universal model completions.

Definition 6.7. Let $T_{0} \subseteq T$ be theories over the respective signatures $\Omega_{0} \subseteq$ $\Omega_{1}$. We say that $T$ is $T_{0}$-positive-universally compatible iff $T_{0}$ is universal, has a positive-universal model completion $T_{0}^{*}$, and every model of $T$ embeds into a model of $T \cup T_{0}^{*}$.

If the prerequisites of Theorem 4.1 hold and $T_{1}$ is additionally $T_{0}$-positive-universally compatible, then decidability of the universal fragment transfers from $T_{1}, T_{2}$ to $T_{1}>_{T_{0}}^{s} T_{2}$.

THEOREM 6.8. Let $T_{0}, T_{1}$, and $T_{2}$ be theories over the respective signatures $\Omega_{0}, \Omega_{1}$, and $\Omega_{2}$, where $\Omega_{0}$ is a common subsignature of $\Omega_{1}$ and $\Omega_{2}$. Assume that $T_{0} \subseteq T_{1}$ and $T_{0} \subseteq T_{2}$, that $T_{0}$ is universal and locally finite, that $T_{1}$ is $T_{0}$-positiveuniversally compatible, and that $T_{2}$ is $T_{0}$-positive-existentially compatible. Then 
the decidability of the universal fragments of $T_{1}$ and $T_{2}$ entails the decidability of the universal fragment of $T_{1}>_{T_{0}}^{s} T_{2}$.

To prove the theorem, let $\Gamma=\Gamma_{0} \cup \Gamma_{1} \cup \Gamma_{2}$ be a finite set of ground flat literals over the signature $\Omega$ of $T_{1}>_{T_{0}}^{s} T_{2}$ (with additional free constants), where $\Gamma_{i}$ $(i=1,2)$ is a set of literals in the signature $\Omega_{i}$ (expanded with free constants), and $\Gamma_{0}$ is of the form

$$
\left\{h\left(a_{1}\right) \approx b_{1}, \ldots, h\left(a_{n}\right) \approx b_{n}\right\},
$$

for free constants $a_{1}, b_{1}, \ldots, a_{n}, b_{n}$. The following proposition, whose formulation is identical to the formulation of Proposition 4.2, immediately entails Theorem 6.8 .

Proposition 6.9. The constraint $\Gamma=\Gamma_{0} \cup \Gamma_{1} \cup \Gamma_{2}$ is satisfiable in $T_{1}>_{T_{0}}^{s} T_{2}$ iff there exists a triple $(\mathcal{A}, \mathcal{B}, \nu)$ such that

1. $\mathcal{A}$ is an $\Omega_{0}$-model of $T_{0}$, which is generated by $\left\{a_{1}^{\mathcal{A}}, \ldots, a_{n}^{\mathcal{A}}\right\}$;

2. $\mathcal{B}$ is an $\Omega_{0}$-model of $T_{0}$, which is generated by $\left\{b_{1}^{\mathcal{B}}, \ldots, b_{n}^{\mathcal{B}}\right\}$;

3. $\nu: \mathcal{A} \rightarrow \mathcal{B}$ is an $\Omega_{0}$-homomorphism such that $\nu\left(a_{j}^{\mathcal{A}}\right)=b_{j}^{\mathcal{B}}$ for $j=1, \ldots, n$;

4. $\Gamma_{1} \cup \Delta_{\Omega_{0}}(\mathcal{A})$ is satisfiable in $T_{1}$;

5. $\Gamma_{2} \cup \Delta_{\Omega_{0}}(\mathcal{B})$ is satisfiable in $T_{2}$.

Proof. The only-if direction is again simple. The proof of the if direction requires now a back-and-forth argument. Suppose we are given $\mathcal{A}, \mathcal{B}, \nu$ as in 1. -5 . of the proposition, and let $\mathcal{N}^{\prime}$ be an $\Omega_{1}$-model of $T_{1}$ satisfying $\Gamma_{1} \cup \Delta_{\Omega_{0}}(\mathcal{A})$, and $\mathcal{N}^{\prime \prime}$ be an $\Omega_{2}$-model of $T_{2}$ satisfying $\Gamma_{2} \cup \Delta_{\Omega_{0}}(\mathcal{B})$. We can assume without loss of generality that $\mathcal{N}^{\prime}, \mathcal{N}^{\prime \prime}$ are both at most countable, that $\mathcal{N}^{\prime}$ is a model of the positive-universal model completion of $T_{0}$, and that $\mathcal{N}^{\prime \prime}$ is a model of the positive-existential model completion of $T_{0}$. By Robinson's diagram theorem, $\mathcal{N}^{\prime}$ has $\mathcal{A}$ as an $\Omega_{0}$-substructure, and $\mathcal{N}^{\prime \prime}$ has $\mathcal{B}$ as an $\Omega_{0}$-substructure. Let us enumerate the elements of $N^{\prime}$ as

$$
c_{1}, c_{3}, \ldots, c_{2 k+1}, \ldots
$$

and the elements of $N^{\prime \prime}$ as

$$
d_{2}, d_{4}, \ldots, d_{2 k}, \ldots
$$

(here we prefer, for uniformity, both lists to be infinite, so we may tolerate repetitions in each list). We define an increasing sequence of sort-conforming surjective mappings $\nu_{k}: S_{k} \rightarrow T_{k}$, such that:

- $S_{k}$ is a finite subset of $N^{\prime}$ including all the elements from $A$ as well as $c_{2 j+1}$, for $2 j+1 \leq k$;

- $T_{k}$ is a finite subset of $N^{\prime \prime}$ including all the elements from $B$ as well as $d_{2 j}$, for $2 j \leq k$;

- for all $\Omega_{0}$-atoms $C(\underline{x})$ we have

$$
\mathcal{N}_{\mid \Omega_{0}}^{\prime}=C(\underline{a}) \quad \text { implies } \quad \mathcal{N}_{\mid \Omega_{0}}^{\prime \prime} \models C\left(\nu_{k}(\underline{a})\right)
$$

for every tuple $\underline{a}$ from $S_{k}$.

Once this is settled, $\mathcal{N}^{\prime}$ and $\mathcal{N}^{\prime \prime}$ together with the surjective homomorphism $\nu_{\infty}=\bigcup_{k \geq n} \nu_{k}$ give, as usual, the desired model of $T_{1}>_{T_{0}}^{s} T_{2}$ satisfying $\Gamma$. 
We first take $\nu_{0}$ to be $\nu$. To define $\nu_{k}(k>0)$, we distinguish the case in which $k$ is even from the case in which $k$ is odd. In the latter case, we proceed as in the proof of Proposition 4.2. As to the former case, let $b=d_{2 k}$ and let $\underline{a}$ be a tuple collecting all the elements from $S_{k-1}$. We want to find a suitable $a \in N^{\prime}$ in order to extend $\nu_{k-1}$ by defining $\nu_{k}(a):=b$. For this purpose, it is sufficient to show that $\mathcal{N}^{\prime} \not \forall \forall y . \phi(\underline{a}, y)$, where $\phi(\underline{x}, y)$ is the disjunction of all atoms $C(\underline{x}, y)$ such that $\mathcal{N}^{\prime \prime} \not \models C\left(\nu_{k-1}(\underline{a}), b\right)$. In fact, if $\mathcal{N}^{\prime} \not \forall \forall y . \phi(\underline{a}, y)$, then there is a (sort-conforming) $a \in N^{\prime}$ such that $\mathcal{N}^{\prime} \models \neg \phi(\underline{a}, a)$, and we can set $\nu_{k}(a):=b$. Assume that $C$ is an atom such that $\mathcal{N}_{\mid \Omega_{0}}^{\prime} \models C(\underline{a}, a)$, but $\mathcal{N}_{\mid \Omega_{0}}^{\prime \prime} \not \models C\left(\nu_{k}(\underline{a}, a)\right)=C\left(\nu_{k-1}(\underline{a}), b\right)$. However, this means that $C(\underline{x}, y)$ occurs as a disjunct in $\phi(\underline{x}, y)$, and thus $\mathcal{N}^{\prime} \models \neg \phi(\underline{a}, a)$ implies that $\mathcal{N}^{\prime} \models \neg C(\underline{a}, a)$, which is a contradiction to our assumption that $\mathcal{N}_{\mid \Omega_{0}}^{\prime} \models C(\underline{a}, a)$.

To show that $\mathcal{N}^{\prime} \not \forall \forall y . \phi(\underline{a}, y)$, we consider the positive-universal model completion $T_{0}^{*}$ of $T_{0}$. In this theory, $\forall y . \phi(\underline{x}, y) \leftrightarrow \phi^{*}(\underline{x})$ is provable for some co-geometric (and thus also geometric ${ }^{12}$ ) open formula $\phi^{*}(\underline{x})$. As usual, the implication $\phi^{*}(\underline{x}) \rightarrow \forall y . \phi(\underline{x}, y)$ must already hold in $T_{0}$ because $T_{0}$ and its positive-universal model completion $T_{0}^{*}$ entail the same open formulae, and $\phi^{*}(\underline{x}) \rightarrow \forall y . \phi(\underline{x}, y)$ is equivalent to the open formula $\phi^{*}(\underline{x}) \rightarrow \phi(\underline{x}, y)$.

Since $\mathcal{N}^{\prime}$ is a model of $T_{0}^{*}$, and $T_{0}^{*} \models \forall y . \phi(\underline{x}, y) \rightarrow \phi^{*}(\underline{x})$, it is enough to prove that $\mathcal{N}^{\prime} \not \models \phi^{*}(\underline{a})$. However, $\mathcal{N}^{\prime \prime} \not \forall \forall y . \phi\left(\nu_{k-1}(\underline{a}), y\right)$, by the definition of $\phi$. Since $\mathcal{N}^{\prime \prime}$ is a model of $T_{0}$, and $T_{0} \models \phi^{*}(\underline{x}) \rightarrow \forall y . \phi(\underline{x}, y)$, this implies $\mathcal{N}^{\prime \prime} \not \forall \phi^{*}\left(\nu_{k-1}(\underline{a})\right)$. Finally, the induction hypothesis on the validity of (12) yields $\mathcal{N}^{\prime} \not \models \phi^{*}(\underline{a})$.

The following example shows that there are natural theories $T_{0}$ admitting both a positive-existential and a positive-universal model completion.

EXAMPLE 6.10. Consider the theory of join semilattices with a greatest element. These are join semilattices as introduced in Example 4.6, but endowed with a further element $\top$ such that $x \sqcup \top=\top$ holds for all $x$. The positiveexistential model completion of this theory is axiomatized as in Example 4.6 above. In order to axiomatize the positive-universal model completion of this theory, we need a theory that allows us to eliminate the universal quantifier from formulae $\forall y . \phi(\underline{x}, y)$ of the form

$$
\forall y .\left(\left(y \sqsubseteq t_{1}\right) \vee \cdots \vee\left(y \sqsubseteq t_{n}\right) \vee\left(u_{1} \sqsubseteq s_{1} \sqcup y\right) \vee \cdots \vee\left(u_{m} \sqsubseteq s_{m} \sqcup y\right)\right),
$$

where $t_{i}, s_{j}, u_{k}$ are terms not involving $y$. Let $\phi^{*}(\underline{x})$ be the formula

$$
\bigvee_{i=1}^{n}\left(t_{i} \approx \top\right) \vee \bigvee_{j=1}^{m}\left(u_{j} \sqsubseteq s_{j}\right),
$$

and let $T_{0}^{*}$ be obtained from $T_{0}$ by adding to it the universal closures of the sentences $\phi \leftrightarrow \phi^{*}$. The theory $T_{0}^{*}$ is included in the theory $B A^{*}$ of atomless Boolean algebras (recall that a Boolean algebra is said to be atomless iff it does not have non-zero minimal elements): the axioms of $T_{0}^{*}$ are in fact provable in $B A^{*}$, as it is evident from the quantifier elimination procedure for $B A^{*}$ (see, e.g., [17]). Since every join semilattice with a greatest element embeds into an

\footnotetext{
${ }^{12}$ In the open case, geometric and co-geometric formulae trivially coincide.
} 
atomless Boolean algebra, ${ }^{13}$ this shows both that $T_{0}^{*}$ is the positive-universal model completion of $T_{0}$, and that the theory of Boolean algebras is positive-universally compatible with the theory of join semilattices with a greatest element.

Since the formulation of Proposition 6.9 coincides with the one of Proposition 4.2 , we know that the universal fragments of $T_{1}>_{T_{0}}^{s} T_{2}$ and $T_{1}>_{T_{0}} T_{2}$ coincide if the conditions of Theorem 6.8 are satisfied.

COROLlary 6.11. Let $T_{0}, T_{1}$, and $T_{2}$ be theories over the respective signatures $\Omega_{0}, \Omega_{1}$, and $\Omega_{2}$, where $\Omega_{0}$ is a common subsignature of $\Omega_{1}$ and $\Omega_{2}$. Assume that $T_{0} \subseteq T_{1}$ and $T_{0} \subseteq T_{2}$, that $T_{0}$ is universal and locally finite, that $T_{1}$ is $T_{0}$-positiveuniversally compatible, and that $T_{2}$ is $T_{0}$-positive-existentially compatible. Then the universal fragments of $T_{1}>_{T_{0}} T_{2}$ and $T_{1}>_{T_{0}}^{s} T_{2}$ coincide.

6.3. Isomorphisms as connection functions. Finally, let us consider the problem of deciding the universal fragment of $T_{1}>_{T_{0}}^{i s o} T_{2}$.

THEOREM 6.12. Let $T_{0}, T_{1}$, and $T_{2}$ be theories over the respective signatures $\Omega_{0}, \Omega_{1}$, and $\Omega_{2}$, where $\Omega_{0}$ is a common subsignature of $\Omega_{1}$ and $\Omega_{2}$. Assume that $T_{0} \subseteq T_{1}$ and $T_{0} \subseteq T_{2}$, that $T_{0}$ is universal and locally finite, and that $T_{1}, T_{2}$ are both $T_{0}$-compatible. Then the decidability of the universal fragments of $T_{1}$ and $T_{2}$ entails the decidability of the universal fragment of $T_{1}>{ }_{T_{0}}^{i s o} T_{2}$.

This theorem follows from the corresponding theorem for surjective connection functions (Theorem 6.8) due to Proposition 6.3 and the following analogous proposition for the positive-universal case.

Proposition 6.13. Let $T$ be a universal $\Omega$-theory, and $T_{0} \subseteq T$ a theory over the subsignature $\Omega_{0}$ of $\Omega$.

1. $T\urcorner$ has a positive-universal model completion iff $T$ has a model completion.

2. $T\urcorner$ is $\left.T_{0}\right\urcorner$-positive-universally compatible iff $T$ is $T_{0}$-compatible.

Both, the proof of this proposition and the proof of Theorem 6.12, are similar to the corresponding proofs given in Section 6.1. Of course, Theorem 6.12 can again be proved directly. As before, we consider a a finite set of ground flat literals over the signature $\Omega$ of $T_{1}>_{T_{0}}^{i s o} T_{2}$ (with additional free constants), which is of the form $\Gamma=\Gamma_{0} \cup \Gamma_{1} \cup \Gamma_{2}$, where $\Gamma_{i}(i=1,2)$ is a set of literals in the signature $\Omega_{i}$ (expanded with free constants), and $\Gamma_{0}$ is of the form

$$
\left\{h\left(a_{1}\right) \approx b_{1}, \ldots, h\left(a_{n}\right) \approx b_{n}\right\},
$$

for free constants $a_{1}, b_{1}, \ldots, a_{n}, b_{n}$. The following proposition, whose formulation is identical to the formulation of Proposition 6.5, immediately entails Theorem 6.12. The proposition itself is an easy consequence of the Propositions $6.9,6.13$, and 6.3 .

Proposition 6.14. The constraint $\Gamma=\Gamma_{0} \cup \Gamma_{1} \cup \Gamma_{2}$ is satisfiable in $T_{1}>T_{0}^{i s o} T_{2}$ iff there exists a triple $(\mathcal{A}, \mathcal{B}, \nu)$ such that

\footnotetext{
${ }^{13}$ One can embed a join semilattice with greatest element into a bounded distributive lattice by taking the dual of the lattice of non-empty upward closed subsets; that bounded distributive lattices embed into Boolean algebras, and that Boolean algebras embed into atomless Boolean algebras are standard lattice-theoretic facts.
} 
1. $\mathcal{A}$ is an $\Omega_{0}$-model of $T_{0}$, which is generated by $\left\{a_{1}^{\mathcal{A}}, \ldots, a_{n}^{\mathcal{A}}\right\}$;

2. $\mathcal{B}$ is an $\Omega_{0}$-model of $T_{0}$, which is generated by $\left\{b_{1}^{\mathcal{B}}, \ldots, b_{n}^{\mathcal{B}}\right\}$;

3. $\nu: \mathcal{A} \rightarrow \mathcal{B}$ is an $\Omega_{0}$-embedding such that $\nu\left(a_{j}^{\mathcal{A}}\right)=b_{j}^{\mathcal{B}}$ for $j=1, \ldots, n$;

4. $\Gamma_{1} \cup \Delta_{\Omega_{0}}(\mathcal{A})$ is satisfiable in $T_{1}$;

5. $\Gamma_{2} \cup \Delta_{\Omega_{0}}(\mathcal{B})$ is satisfiable in $T_{2}$.

Since the formulation of Proposition 6.14 coincides with the one of Proposition 6.5 , we know that the universal fragments of $T_{1}>_{T_{0}}^{e m} T_{2}$ and $T_{1}>_{T_{0}}^{i s o} T_{2}$ coincide if the conditions of Theorem 6.12 are satisfied.

COROLlary 6.15. Let $T_{0}, T_{1}$, and $T_{2}$ be theories over the respective signatures $\Omega_{0}, \Omega_{1}$, and $\Omega_{2}$, where $\Omega_{0}$ is a common subsignature of $\Omega_{1}$ and $\Omega_{2}$. Assume that $T_{0} \subseteq T_{1}$ and $T_{0} \subseteq T_{2}$, that $T_{0}$ is universal and locally finite, and that $T_{1}, T_{2}$ are $T_{0}$-compatible. Then the universal fragment of $T_{1}>_{T_{0}}^{e m} T_{2}$ coincides with the universal fragment of $T_{1}>{ }_{T_{0}}^{i s o} T_{2}$.

It is easy to see that the problem of deciding the universal fragment of $T_{1}>_{T_{0}}^{i s o}$ $T_{2}$ is interreducable in polynomial time with the problem of deciding the universal fragment of $T_{1} \cup T_{2}$. Consequently, the proof of Theorem 6.12 yields an alternative proof of the combination result in [16].

The main reason for this is that there is a close connection between models of $T_{1} \cup T_{2}$ and $T_{1}>_{T_{0}}^{i s o} T_{2}$. In fact, if $\mathcal{M}$ is a model of $T_{1} \cup T_{2}$, then it can be turned into a model $\left(\mathcal{M}^{1}, \mathcal{M}^{2}, \nu\right)$ of $T_{1}>_{T_{0}}^{\text {iso }} T_{2}$ by taking as $\mathcal{M}^{1}$ the reduct of $\mathcal{M}$ to $\Omega_{1}$, as $\mathcal{M}^{2}$ the reduct of $\mathcal{M}$ to $\Omega_{2}$, and as isomorphism $\nu$ the identity mapping on the domain of the reduct of $\mathcal{M}$ to $\Omega_{0}$. Conversely, if $\left(\mathcal{M}^{1}, \mathcal{M}^{2}, \nu\right)$ is a model of $T_{1}>{ }_{T_{0}}^{i s o} T_{2}$, then one can turn it into a model of $T_{1} \cup T_{2}$ by adapting the well-known fusion construction [31] to the many-sorted case.

Now, given a conjunction $\Gamma$ of (sort-conforming) literals to be tested for satisfiability in $T_{1}>{ }_{T}^{i s o} T_{2}$, we can simply remove the connection function $h$ and the superscripts introduced through the renaming done in the construction of $T_{1}>\stackrel{\text { iso }}{T_{0}} T_{2}$, and test the resulting conjunction $\Gamma^{\prime}$ of literals for satisfiability in $T_{1} \cup T_{2}$. If $\mathcal{M}$ is a model of $T_{1} \cup T_{2}$ satisfying $\Gamma^{\prime}$, then it is easy to see that the corresponding model $\left(\mathcal{M}^{1}, \mathcal{M}^{2}, \nu\right)$ of $T_{1}>_{T_{0}}^{\text {iso }} T_{2}$ satisfies $\Gamma$. Conversely, if $\left(\mathcal{M}^{1}, \mathcal{M}^{2}, \nu\right)$ is a model of $T_{1}>_{T_{0}}^{i s o} T_{2}$ satisfying $\Gamma$, then it is easy to see that the model $\mathcal{M}$ of $T_{1} \cup T_{2}$ obtained from this model by applying the fusion construction satisfies $\Gamma^{\prime}$.

Conversely, given a conjunction $\Gamma$ of ground flat literals to be tested for satisfiability in $T_{1} \cup T_{2}$, we can partition $\Gamma$ into $\Gamma=\Gamma_{1} \cup \Gamma_{2}$ where $\Gamma_{1}$ is over the signature $\Omega_{1}$ and $\Gamma_{2}$ is over the signature $\Omega_{2}$. For every free constant $c$ occurring in $\Gamma$, we introduce two free constants $c^{1}$ and $c^{2}$. We replace $c$ in $\Gamma_{1}$ by $c^{1}$ and $c$ in $\Gamma_{2}$ by $c^{2}$, and also do the appropriate renamings of the shared function and predicate symbols. In addition, we add the equation $c^{2} \approx h\left(c^{1}\right)$ for each free constant $c$ occurring in $\Gamma$. Let $\Gamma^{\prime}$ be the conjunction of literals over the signature of $T_{1}>{ }_{T_{0}}^{i s o} T_{2}$ obtained this way. Again, it is easy to see that $\Gamma$ is satisfiable in $T_{1} \cup T_{2}$ iff $\Gamma^{\prime}$ is satisfiable in $T_{1}>_{T_{0}}^{i s o} T_{2}$.

COROLlary 6.16. Let $T_{0}, T_{1}$, and $T_{2}$ be theories over the respective signatures $\Omega_{0}, \Omega_{1}$, and $\Omega_{2}$, where $\Omega_{0}$ is a common subsignature of $\Omega_{1}$ and $\Omega_{2}$. Assume that $T_{0} \subseteq T_{1}$ and $T_{0} \subseteq T_{2}$, that $T_{0}$ is universal and locally finite, and that $T_{1}, T_{2}$ are 
both $T_{0}$-compatible. Then the decidability of the universal fragments of $T_{1}$ and $T_{2}$ entails the decidability of the universal fragment of $T_{1} \cup T_{2}$.

$\S 7$. The connection to $\mathcal{E}$-connections. The purpose of this section is to give a more detailed comparison between the notion of an $\mathcal{E}$-connection, as introduced in [20], and our notion of a connection of many-sorted theories.

First of all, note that [20] consider connections that are more general than ours, in the sense that more complex modalities ( $n$-ary modalities, inverse modalities, Boolean combinations of modalities, counting modalities, etc.) can be used as connection functions. Using such sophisticated modalities as connection function is, currently, beyond the scope of our methods, but they will be the subject of future research.

Here, we will content ourselves with examining the special case of plain unary modalities as connection functions, which is the most basic case of an $\mathcal{E}$-connection considered in [20]. However, even with this restriction, there are still significant differences between our approach and the approach in [20]. The main difference is that, seen from the modal logic point of view, our approach for defining the connection is syntactic (or algebraic), in the sense that we consider an equational axiomatization of the logic. In contrast, in [20] the emphasis is on the modeltheoretic side, meaning that $\mathcal{E}$-connections are defined at the semantic level as enrichments of suitable Kripke-like structures. Because of this difference, it is not a priori clear that our results specialize to decidability transfer results for $\mathcal{E}$ connections defined in the framework of [20] (even within the limitation to plain unary modalities as connection functions). In this section, we show that this is indeed the case (but this proof turns out to be not entirely trivial). To simplify matters further, we will not consider abstract description systems in their full generality (as used in [20] as the components of $\mathcal{E}$-connections), but restrict our considerations to normal modal logics and to standard uni-modal Kripke frames (most of these further restrictions are, however, without loss of generality; they are assumed just for the sake of simplicity of presentation).

Propositional modal formulae are built using the Boolean connectives and a diamond operator $\diamond$. A Kripke frame is a pair $\mathcal{F}=(W, R)$, where $W$ is a non-empty set, the set of possible worlds, and $R$ is a binary relation on $W$, the transition relation. A Kripke model is a triple $\mathcal{M}=(W, R, V)$, where $(W, R)$ is a Kripke frame and $V$ is a map, called valuation, associating with each propositional letter a subset of $W$. The forcing relation $w=^{\mathcal{M}} \alpha$, which expresses that the modal formula $\alpha$ is true in the Kripke model $\mathcal{M}$ at world $w$, is defined in the standard way (see, e.g., [9]).

For a given class of Kripke frames $\mathcal{C}$, the modal constraint problem for $\mathcal{C}$ is the problem of deciding whether a finite set of modal formulae is satisfiable w.r.t. a set of global constraints. ${ }^{14}$

\footnotetext{
${ }^{14}$ This is the kind of problem considered in [20], where satisfiability of an A-Box containing many individual constants, with respect to a given T-Box, is taken into consideration. Notice that, by contrast, relativized satisfiability as traditionally considered in modal logic concerns local satisfiability of just one formula with respect to a global constraint formed by a finite set of formulae, i.e., is is the special case of modal constraints for which $m=1$.
} 
Definition 7.1. A modal constraint is a pair of finite sets of modal formulae, written as $\alpha_{1}, \ldots, \alpha_{n} ; \beta_{1}, \ldots, \beta_{m}(n, m \geq 0)$; we say that such a modal constraint is satisfiable in a Kripke model $\mathcal{M}=(W, R, V)$ iff there are worlds $w_{1}, \ldots, w_{m} \in$ $W$ such that

1. $w_{1} \models^{\mathcal{M}} \beta_{1}, \ldots, w_{m} \models^{\mathcal{M}} \beta_{m}$;

2. for all $v \in W$ and for all $i=1, \ldots, n$, we have $v \models{ }^{\mathcal{M}} \alpha_{i}$.

The modal constraint $\alpha_{1}, \ldots, \alpha_{n} ; \beta_{1}, \ldots, \beta_{m}$ is satisfiable in a class of Kripke frames $\mathcal{C}$ iff it is satisfiable in some $\mathcal{M}=(W, R, V)$, for $(W, R) \in \mathcal{C}$.

Thus, the satisfiability of a modal constraint $\alpha_{1}, \ldots, \alpha_{n} ; \beta_{1}, \ldots, \beta_{m}$ means that there is a model in which the $\beta_{j}$ are satisfied in some worlds $w_{j}$, and in which $\alpha_{1}, \ldots, \alpha_{n}$ hold globally, i.e., in every world.

In order to algebraize the above decision problem, let us introduce the signature $B_{M}$ : this is the single-sorted signature obtained by expanding the signature of Boolean algebras by a new unary operator that we still call $\diamond$. Notice that there is an obvious bijective correspondence in this way between modal formulae and terms of the signature $B_{M}$ (thus, from now on, we identify modal formulae and terms of the signature $\left.B_{M}\right)$. Also, a Kripke frame $\mathcal{F}=(W, R)$ can be converted into a $B_{M}$-structure called $\mathcal{B}_{\mathcal{F}}$ as follows: we take as underlying Boolean algebra the powerset Boolean algebra $\mathcal{P}(W)$ and interpret $\diamond$ as the function associating with $X \subseteq W$ the subset of $W$ given by

$$
\diamond(X):=\left\{w_{2} \in W \mid \exists w_{1} \in W .\left(w_{2}, w_{1}\right) \in R \wedge w_{1} \in X\right\} .
$$

Valuations $V$ of $\mathcal{F}$ correspond in an obvious way to assignments of variables to elements of $\mathcal{P}(W)$. It is easy to see that, for any modal formula $\theta$, we have $w \models^{(W, R, V)} \theta$ iff $w$ belongs to the set obtained by evaluating the term $\theta$ in $\mathcal{B}_{\mathcal{F}}$ under the assignment $V$.

With every class of Kripke frames $\mathcal{C}$ we associate the $B_{M}$-theory $T_{\mathcal{C}}$ whose axioms are the formulae

$$
\left(\alpha_{1} \approx \top\right) \wedge \cdots \wedge\left(\alpha_{n} \approx \top\right) \rightarrow\left(\beta_{1} \approx \perp\right) \vee \cdots \vee\left(\beta_{m} \approx \perp\right),
$$

where $\alpha_{1}, \ldots, \alpha_{n} ; \beta_{1}, \ldots, \beta_{m}$ are the modal constraints that are not satisfiable in $\mathcal{C}$. If $\mathcal{F}$ is a Kripke frame in $\mathcal{C}$, then the corresponding $B_{M}$-structure $\mathcal{B}_{\mathcal{F}}$ is a model of $T_{\mathcal{C}}$.

Proposition 7.2. The problem of deciding satisfiability of modal constraints in $\mathcal{C}$ is equivalent to the problem of deciding the universal fragment of the theory $T_{\mathcal{C}}$.

Proof. First, notice that a modal constraint

$$
\alpha_{1}, \ldots, \alpha_{n} ; \beta_{1}, \ldots, \beta_{m}
$$

is unsatisfiable in $\mathcal{C}$ iff the formula (15) is a logical consequence of $T_{\mathcal{C}}$. In fact, if (16) is unsatisfiable in $\mathcal{C}$, then (15) is an axiom of $T_{\mathcal{C}}$. Conversely, if (16) is satisfiable in a frame $\mathcal{F}=(W, R) \in \mathcal{C}$, then (15) cannot be a logical consequence of $T_{\mathcal{C}}$, because it it is easy to see that it is then false in the $B_{M}$-structure $\mathcal{B}_{\mathcal{F}}$.

Given that, it is sufficient to observe that identities in $T_{\mathcal{C}}$ are all equivalent ${ }^{15}$ to identities of the kind $\alpha \approx \top$ as well as to identities of the kind $\beta \approx \perp$.

\footnotetext{
${ }^{15}$ Use Boolean bi-implication and complement to show this.
} 
Thus an arbitrary open formula in the signature $B_{M}$ is in fact a conjunction of formulae of the kind (15). Together with what we have shown about the connection between such formulae and modal constraints, this implies the claim of the proposition.

Let us now show that this correspondence

$$
\mathcal{C} \longmapsto T_{\mathcal{C}}
$$

is compatible with building connections, where on the left-hand side the connections are the $\mathcal{E}$-connections as introduced in [20], and on the right-hand side the connections are the connections of many-sorted theories as introduced in the present paper. To show this, we need to recall the definition of an $\mathcal{E}$-connection (in the present simplified case of classes of Kripke frames).

For $\mathcal{E}$-connections, we use two-sorted propositional modal formulae. The formulae of sort 1 are just the standard propositional modal formulae (where, however, the modal operator $\diamond$ is renamed to $\diamond_{1}$ ); the formulae of sort 2 are built from propositional variables ${ }^{16}$ of sort 2 and formulae of the form $\diamond_{E} \phi$ where $\phi$ is a formula of sort 1, by applying the Boolean connectives and the modal operator $\diamond_{2}$.

From the semantic side, suppose we are given two classes $\mathcal{C}_{1}, \mathcal{C}_{2}$ of Kripke frames. The class of connection frames $\mathcal{E}\left(\mathcal{C}_{1}, \mathcal{C}_{2}\right)$ is formed by all triples $\mathcal{F}=$ $\left(\mathcal{F}_{1}, E^{\mathcal{F}}, \mathcal{F}_{2}\right)$ such that $\mathcal{F}_{1}=\left(W_{1}, R_{1}\right) \in \mathcal{C}_{1}, \mathcal{F}_{2}=\left(W_{2}, R_{2}\right) \in \mathcal{C}_{2}$ and $E^{\mathcal{F}} \subseteq$ $W_{2} \times W_{1}$ is an arbitrary binary relation.

An $\mathcal{E}\left(\mathcal{C}_{1}, \mathcal{C}_{2}\right)$-connection Kripke model is a 4-tuple $\mathcal{M}=\left(\mathcal{F}_{1}, E^{\mathcal{F}}, \mathcal{F}_{2}, V\right)$, where $\mathcal{F}=\left(\mathcal{F}_{1}, E^{\mathcal{F}}, \mathcal{F}_{2}\right) \in \mathcal{E}\left(\mathcal{C}_{1}, \mathcal{C}_{2}\right)$ is a connection frame and $V$ is a map associating with propositional letters of sort $i$ subsets of $W_{i}(i=1,2)$. The forcing relation $w \models{ }^{\mathcal{M}} \alpha$, which says that the modal formula $\alpha$ is true in $\mathcal{M}$ at world $w$, is defined in the standard way (see [20]), where the only non-obvious case is the following: for $w_{2} \in W_{2}$ and for a formula $\alpha$ of sort 1 , we have:

$$
w_{2} \models{ }^{\mathcal{M}} \diamond_{E} \alpha \quad \text { iff } \quad\left(\exists w_{1} \in W_{1} .\left(w_{2}, w_{1}\right) \in E^{\mathcal{F}} \text { and } w_{1} \models^{\mathcal{M}} \alpha\right) .
$$

Now, $\mathcal{E}\left(\mathcal{C}_{1}, \mathcal{C}_{2}\right)$-satisfiability of a modal constraint $\alpha_{1}, \ldots, \alpha_{n} ; \beta_{1}, \ldots, \beta_{m}$ is defined as above (but notice that the $\alpha_{i}$ and the $\beta_{j}$ may be formulae of sort 1 or 2 , indifferently).

When connecting the theories corresponding to two frame classes, we build the two-sorted signature $B_{M}^{2}$ : this consists of two renamed copies of the signature $B_{M}$ and, in addition, of the new unary function symbol $\diamond_{E}$ of arity $S_{1} S_{2}$ (where $S_{1}, S_{2}$ are the single sorts of the renamed copies of $B_{M}$ ). Again, terms in the signature $B_{M}^{2}$ can be identified with the two-sorted modal formulae introduced above; moreover any connection frame $\mathcal{F}=\left(\mathcal{F}_{1}, E^{\mathcal{F}}, \mathcal{F}_{2}\right)$ can be turned into a $B_{M}^{2}$-structure (which we still call $\mathcal{B}_{\mathcal{F}}$ ) by interpreting the two sorts by powerset Boolean algebras, as described above, and by defining $\diamond_{E}$ as the function associating with $X \subseteq W_{1}$ the subset of $W_{2}$ given by

$$
\diamond_{E}(X):=\left\{w_{2} \in W_{2} \mid \exists w_{1} \in W .\left(w_{2}, w_{1}\right) \in E^{\mathcal{F}} \wedge w_{1} \in X\right\} .
$$

${ }^{16}$ Propositional variables of sort 1 are kept disjoint from propositional variables of sort 2. 
We can then build the theory $T_{\mathcal{E}\left(\mathcal{C}_{1}, \mathcal{C}_{2}\right)}$, whose axioms are the formulae

$$
\left(\alpha_{1} \approx \top\right) \wedge \cdots \wedge\left(\alpha_{n} \approx \top\right) \rightarrow\left(\beta_{1} \approx \perp\right) \vee \cdots \vee\left(\beta_{m} \approx \perp\right),
$$

where $\alpha_{1}, \ldots, \alpha_{n} ; \beta_{1}, \ldots, \beta_{m}$ are the modal constraints that are not satisfiable in $\mathcal{E}\left(\mathcal{C}_{1}, \mathcal{C}_{2}\right)$. As in the proof of Proposition 7.2 , it can be shown that the problem of deciding satisfiability of modal constraints in $\mathcal{E}\left(\mathcal{C}_{1}, \mathcal{C}_{2}\right)$ is equivalent to the problem of deciding the universal fragment of the theory $T_{\mathcal{E}\left(\mathcal{C}_{1}, \mathcal{C}_{2}\right)}$.

The following proposition states a precise relationship between $\mathcal{E}$-connections and our connections of many-sorted theories.

Proposition 7.3. Let $\mathcal{C}_{1}, \mathcal{C}_{2}$ be classes of Kripke frames; $T_{\mathcal{E}\left(\mathcal{C}_{1}, \mathcal{C}_{2}\right)}$ coincides with $T_{\mathcal{C}_{1}}>_{T_{0}} T_{\mathcal{C}_{2}}$, where $T_{0}$ is the theory of semilattices. ${ }^{17}$

Proof. Both theories $T_{\mathcal{E}\left(\mathcal{C}_{1}, \mathcal{C}_{2}\right)}$ and $T_{\mathcal{C}_{1}}>_{T_{0}} T_{\mathcal{C}_{2}}$ are universal and relative to the same signature $B_{M}^{2}$, so it is sufficient to show that a finite set of literals is satisfiable in a model of one of them iff it is satisfiable in a model of the other. First, note that a finite set of literals is satisfied in a model of $T_{\mathcal{E}\left(\mathcal{C}_{1}, \mathcal{C}_{2}\right)}$ iff it is satisfied in a model of the form $\mathcal{B}_{\mathcal{F}}$, where $\mathcal{F}=\left(\mathcal{F}_{1}, E^{\mathcal{F}}, \mathcal{F}_{2}\right)$ is such that $\mathcal{F}_{1} \in \mathcal{C}_{1}$ and $\mathcal{F}_{2} \in \mathcal{C}_{2}$. This can be shown by basically repeating the arguments used in the proof of Proposition 7.2: every universal $B_{M}^{2}$-formula is equivalent to conjunction of formulae of the kind (15), and (15) is a logical consequence of the theory $T_{\mathcal{E}\left(\mathcal{C}_{1}, \mathcal{C}_{2}\right)}$ iff the modal constraint (16) is unsatisfiable in frames of the kind $\mathcal{F}=\left(\mathcal{F}_{1}, E^{\mathcal{F}}, \mathcal{F}_{2}\right.$ ) (for $\mathcal{F}_{1} \in \mathcal{C}_{1}$ and $\mathcal{F}_{2} \in \mathcal{C}_{2}$ ), i.e., iff (15) is true in models of the kind $\mathcal{B}_{\mathcal{F}}$, where $\mathcal{F}=\left(\mathcal{F}_{1}, E^{\mathcal{F}}, \mathcal{F}_{2}\right)$ is such that $\mathcal{F}_{1} \in \mathcal{C}_{1}$ and $\mathcal{F}_{2} \in \mathcal{C}_{2}$.

Clearly, models of the form $\mathcal{B}_{\mathcal{F}}$ for a connection frame $\mathcal{F}=\left(\mathcal{F}_{1}, E^{\mathcal{F}}, \mathcal{F}_{2}\right)$ are models of $T_{\mathcal{C}_{1}}>_{T_{0}} T_{\mathcal{C}_{2}}$. However, the converse is far from being true: in fact, models of $T_{\mathcal{C}_{1}}>_{T_{0}} T_{\mathcal{C}_{2}}$ may interpret the two sorts $S_{1}$ and $S_{2}$ by Boolean algebras that are not powerset Boolean algebras. Moreover, in models of $T_{\mathcal{C}_{1}}>_{T_{0}} T_{\mathcal{C}_{2}}$, the connecting diamond $\diamond_{E}$ is taken to be any semilattice homomorphism and, as such, it need not preserve infinitary joins (as is the case, on the contrary, for the interpretation of $\diamond_{E}$ in all models of the kind $\mathcal{B}_{\mathcal{F}}$ ).

Thus, the key point of the proof is to show that any finite set of $B_{M}^{2}$-literals $\Gamma$ satisfiable in a model of $T_{\mathcal{C}_{1}}>_{T_{0}} T_{\mathcal{C}_{2}}$, is also satisfiable in a model of the form $\mathcal{B}_{\mathcal{F}}$, where $\mathcal{F}=\left(\mathcal{F}_{1}, E^{\mathcal{F}}, \mathcal{F}_{2}\right)$ is a connection frame such that $\mathcal{F}_{1} \in \mathcal{C}_{1}$ and $\mathcal{F}_{2} \in \mathcal{C}_{2}$.

We can, as usual, replace variables with constants and assume $\Gamma$ to be flat, so that we can divide $\Gamma$ into three disjoint sets $\Gamma=\Gamma_{0} \cup \Gamma_{1} \cup \Gamma_{2}$, where $\Gamma_{i}$ $(i=1,2)$ is a set of literals in the $i$-th copy of the signature $B_{M}$ (expanded with free constants), and $\Gamma_{0}$ is of the form

$$
\Gamma_{0}=\left\{\diamond_{E}\left(a_{1}\right) \approx b_{1}, \ldots, \diamond_{E}\left(a_{n}\right) \approx b_{n}\right\}
$$

for free constants $a_{1}, b_{1}, \ldots, a_{n}, b_{n}$.

This observation is not sufficient yet: we need to modify $\Gamma_{0} \cup \Gamma_{1} \cup \Gamma_{2}$ further. Let $\Theta$ be the set of terms of the form

$$
\pm a_{1} \sqcap \cdots \sqcap \pm a_{n},
$$

where $+a_{i}$ is $a_{i}$ and $-a_{i}$ is $\overline{a_{i}}$. Notice that the equations

$$
a_{i} \approx \bigsqcup\left\{\theta \mid \theta \in \Theta, \theta \sqsubseteq a_{i}\right\}
$$

\footnotetext{
${ }^{17}$ See Example 2.2.
} 
are logical consequence of the Boolean algebra axioms, and hence are always valid in our models (here $\theta \sqsubseteq a_{i}$ means that $a_{i}$ (and not $\overline{a_{i}}$ ) appears as conjunct in $\theta$ ).

Let $\tilde{\Gamma}_{1}$ be any set of $B_{M}^{1}$-literals obtained from $\Gamma_{1}$ by adding either $\theta \approx \perp$ or $\theta \not \nsim \perp$ for every $\theta \in \Theta$. For any $\theta \in \Theta$, introduce a new constant $c_{\theta}$ and replace $\Gamma_{0}$ with

Finally, let

$$
\tilde{\Gamma}_{0}:=\left\{\diamond_{E}(\theta) \approx c_{\theta} \mid \theta \in \Theta\right\}
$$

$$
\tilde{\Gamma}_{2}\left(\tilde{\Gamma}_{1}\right):=\Gamma_{2} \cup\left\{c_{\theta} \approx \perp \mid \theta \approx \perp \in \tilde{\Gamma}_{1}\right\} \cup\left\{\left(\bigsqcup_{\theta \sqsubseteq a_{i}} c_{\theta}\right) \approx b_{i} \mid i=1, \ldots, n\right\} .
$$

It is easily seen that $\Gamma_{0} \cup \Gamma_{1} \cup \Gamma_{2}$ is satisfiable in a model of $T_{\mathcal{C}_{1}}>_{T_{0}} T_{\mathcal{C}_{2}}$ iff there is a $\tilde{\Gamma}_{1}$ such that $\tilde{\Gamma}_{0} \cup \tilde{\Gamma}_{1} \cup \tilde{\Gamma}_{2}\left(\tilde{\Gamma}_{1}\right)$ is satisfiable in a model of $T_{\mathcal{C}_{1}}>_{T_{0}} T_{\mathcal{C}_{2}}$. The same observation applies to satisfiability in models of $T_{\mathcal{E}\left(\mathcal{C}_{1}, \mathcal{C}_{2}\right)}$. So, let us fix a set $\tilde{\Gamma}_{0} \cup \tilde{\Gamma}_{1} \cup \tilde{\Gamma}_{2}\left(\tilde{\Gamma}_{1}\right)$, and assume that it is satisfiable in a model of $T_{\mathcal{C}_{1}}>_{T_{0}} T_{\mathcal{C}_{2}}$. We must show that it is satisfiable in a model of $T_{\mathcal{E}\left(\mathcal{C}_{1}, \mathcal{C}_{2}\right)}$.

Now, if $\tilde{\Gamma}_{0} \cup \tilde{\Gamma}_{1} \cup \tilde{\Gamma}_{2}\left(\tilde{\Gamma}_{1}\right)$ is satisfiable in a model of $T_{\mathcal{C}_{1}}>_{T_{0}} T_{\mathcal{C}_{2}}$, then $\tilde{\Gamma}_{1}$ is satisfiable in a model of $T_{\mathcal{C}_{1}}$ and $\tilde{\Gamma}_{2}\left(\tilde{\Gamma}_{1}\right)$ is satisfiable in a model of $T_{\mathcal{C}_{2}}$. By the definition of $T_{\mathcal{C}_{i}}$, it follows that $\tilde{\Gamma}_{i}$ must be satisfiable in a model of the form $\mathcal{B}_{\mathcal{F}_{i}}$, where $\mathcal{F}_{i}=\left(W_{i}, R_{i}\right) \in \mathcal{C}_{i}(i=1,2)$. So we simply need to define the interpretation $E^{\mathcal{F}}$ of the connecting relation $E$ in such a way that also $\tilde{\Gamma}_{0}$ is satisfied in $\mathcal{F}=\left(\mathcal{F}_{1}, E^{\mathcal{F}}, \mathcal{F}_{2}\right)$. This is done as follows: pick $s_{1} \in W_{1}$ and $s_{2} \in W_{2}$; we say that $\left(s_{2}, s_{1}\right) \in E^{\mathcal{F}}$ iff $s_{2} \in c_{\theta}^{\mathcal{B}_{\mathcal{F}_{2}}},{ }^{18}$ where $\theta$ is the unique element $^{19}$ of $\Theta$ such that $s_{1} \in \theta^{\mathcal{B}_{\mathcal{F}_{1}}}$. This implies that, for every $\theta \in \Theta$, we have $\diamond_{E}^{\mathcal{B}_{\mathcal{F}}}\left(\theta^{\mathcal{B}_{\mathcal{F}_{1}}}\right) \subseteq c_{\theta}^{\mathcal{B}_{\mathcal{F}_{2}}}$. For the converse inclusion, suppose that $s_{2} \in c_{\theta}^{\mathcal{B}_{\mathcal{F}_{2}}}$. Then $\mathcal{B}_{\mathcal{F}_{2}} \forall c_{\theta} \approx \perp$. By the definition of $\tilde{\Gamma}_{2}\left(\tilde{\Gamma}_{1}\right)$ and by the fact that either $\theta \approx \perp \in \tilde{\Gamma}_{1}$ or $\theta \not z \perp \in \tilde{\Gamma}_{1}$, we have that $\mathcal{B}_{\mathcal{F}_{1}} \not \forall \theta \approx \perp$. This means that there is some $s_{1} \in \theta^{\mathcal{B}_{\mathcal{F}_{1}}}$; for such $s_{1}$ we have that $\left(s_{2}, s_{1}\right) \in E^{\mathcal{F}}$, i.e. that $s_{2} \in \diamond_{E}^{\mathcal{B}_{\mathcal{F}}}\left(\theta^{\mathcal{B}_{\mathcal{F}_{1}}}\right)$.

The above proposition, together with our main combination result (Theorem 4.1), and the fact that Boolean-based theories are positive-existentially compatible with respect to the theory of semilattices (Example 4.6), immediately entails the following result:

Corollary 7.4. Let $\mathcal{C}_{1}$ and $\mathcal{C}_{2}$ be classes of modal frames. If the modal constraint problems for $\mathcal{C}_{1}$ and $\mathcal{C}_{2}$ are both decidable, then so is the modal constraint problem for $\mathcal{E}\left(\mathcal{C}_{1}, \mathcal{C}_{2}\right)$.

This decidability transfer result can be proved directly by an argument similar to the one we used to prove Proposition 7.3. Notice, however, that Theorem 4.1 gives in fact more, as it applies to any Boolean-based theory, i.e., also to theories that are not of the kind $T_{\mathcal{C}}$ for a class $\mathcal{C}$ of Kripke frames.

\footnotetext{
${ }^{18}$ We use $t^{\mathcal{B}_{\mathcal{F}_{2}}}$ to denote the interpretation of the ground term $t$ in the structure $\mathcal{B}_{\mathcal{F}_{2}}$ (and similarly for $\mathcal{F}_{1}$ ).

${ }^{19}$ By the definition of $\Theta$, different elements of $\Theta$ are interpreted by disjoint sets in $\mathcal{F}_{1}$, and the union of the interpretations of all elements of $\Theta$ in $\mathcal{F}_{1}$ is $W_{1}$.
} 
Let us now turn to $\mathcal{E}$-connections that correspond to connections of theories where more than the theory of semilattices is shared. The frame classes $\mathcal{E}_{d}\left(\mathcal{C}_{1}, \mathcal{C}_{2}\right)$ and $\mathcal{E}_{f}\left(\mathcal{C}_{1}, \mathcal{C}_{2}\right)$ are defined similarly to $\mathcal{E}\left(\mathcal{C}_{1}, \mathcal{C}_{2}\right)$ : the only difference is that now the connecting relation $E$ is respectively taken to be a partial function and a function. For such deterministic or functional connections, we can show results that are analogous to Proposition 7.3.

Proposition 7.5. Let $\mathcal{C}_{1}$ and $\mathcal{C}_{2}$ be classes of modal frames.

1. $T_{\mathcal{E}_{d}\left(\mathcal{C}_{1}, \mathcal{C}_{2}\right)}$ coincides with $T_{\mathcal{C}_{1}}>_{T_{0}} T_{\mathcal{C}_{2}}$, where $T_{0}$ is the theory of distributive lattices with a least element.

2. $T_{\mathcal{E}_{f}\left(\mathcal{C}_{1}, \mathcal{C}_{2}\right)}$ coincides with $T_{\mathcal{C}_{1}}>_{T_{0}} T_{\mathcal{C}_{2}}$, where $T_{0}$ is the theory of bounded distributive lattices.

Proof. Only slight modifications to the proof of Proposition 7.3 are needed. When building $\tilde{\Gamma}_{2}\left(\tilde{\Gamma}_{1}\right)$, we add also the atoms $c_{\theta_{1}} \sqcap c_{\theta_{2}} \approx \perp$, for $\theta_{1} \neq \theta_{2}$. In the case of a functional connection, we additionally add $T \approx \bigsqcup_{\theta \in \Theta} c_{\theta}$.

To define $E^{\mathcal{F}}$, we now proceed as follows: first, the definition domain of the partial function $E^{\mathcal{F}}$ is $\left(\bigsqcup_{\theta \in \Theta} c_{\theta}\right)^{\mathcal{B}_{\mathcal{F}_{2}}}$. Now notice that any $s_{2}$ in this definition domain belongs to exactly one $c_{\theta}^{\mathcal{B}_{\mathcal{F}_{2}}}$; moreover, if $s_{2} \in c_{\theta}^{\mathcal{B}_{\mathcal{F}_{2}}}$, then $\mathcal{B}_{\mathcal{F}_{2}} \models c_{\theta} \not \approx \perp$ and thus $\mathcal{B}_{\mathcal{F}_{1}} \models \theta \not z \perp$. Select just one $s_{1} \in \theta^{\mathcal{B}_{\mathcal{F}_{1}}}$ and let $E^{\mathcal{F}}\left(s_{2}\right):=s_{1}$. This definition of $E^{\mathcal{F}}$ guarantees that $\mathcal{B}_{\mathcal{F}} \models \diamond_{E} \theta \approx c_{\theta}$ again holds for all $\theta \in \Theta$. In addition, in the case of a functional connection, the presence of $T \approx \bigsqcup_{\theta \in \Theta} c_{\theta}$ in $\tilde{\Gamma}_{2}\left(\tilde{\Gamma}_{1}\right)$ enforces that the definition domain of the partial function $E^{\mathcal{F}}$ is the whole domain.

Given the positive-existential compatibility of any Boolean-based theory with respect to the theory of distributive lattices with a least element and with respect to the theory of bounded distributive lattices (see Examples 4.7 and 4.8), we thus obtain the following decidability transfer results:

COROLlary 7.6. Let $\mathcal{C}_{1}$ and $\mathcal{C}_{2}$ be classes of modal frames. If the modal constraint problems for $\mathcal{C}_{1}$ and $\mathcal{C}_{2}$ are both decidable, then so are the modal constraint problems for $\mathcal{E}_{d}\left(\mathcal{C}_{1}, \mathcal{C}_{2}\right)$ and $\mathcal{E}_{f}\left(\mathcal{C}_{1}, \mathcal{C}_{2}\right)$.

$\S 8$. The theory of theory completions. In this section we develop some model theory concerning the notions of completions of a theories introduced above. The results of this section give further insights into the properties of these important ingredients of our combination approach, though they are not really needed in order to understand and justify our combination procedures. We shall recall well-known classical results for model completions, and show how they can be adapted to the case of positive-existential model completions and positive-universal model completions.

Let us call a model $\mathcal{M}$ of a theory $T$ :

- positive-existentially closed iff every sentence of the kind $\exists \underline{x} \cdot\left(\alpha_{1}(\underline{a}, \underline{x}) \wedge \cdots \wedge\right.$ $\left.\alpha_{n}(\underline{a}, \underline{x})\right)$, where $\underline{a}$ are parameters from $\mathcal{M}$ and the $\alpha_{i}(\underline{y}, \underline{x})$ are atoms, that is satisfied in some $\mathcal{N} \supseteq \mathcal{M}$ with $\mathcal{N} \models T$ is satisfied in $\mathcal{M}$ itself;

- positive-universally closed iff every sentence of the kind $\exists \underline{x} .\left(\neg \alpha_{1}(\underline{a}, \underline{x}) \wedge \cdots \wedge\right.$ $\left.\neg \alpha_{n}(\underline{a}, \underline{x})\right)$, where $\underline{a}$ are parameters from $\mathcal{M}$ and the $\alpha_{i}(y, \underline{x})$ are atoms, that is satisfied in some $\mathcal{N} \supseteq \mathcal{M}$ with $\mathcal{N} \models T$ is satisfied in $\mathcal{M}$ itself; 
- existentially closed iff every sentence of the kind $\exists \underline{x} .\left(\alpha_{1}(\underline{a}, \underline{x}) \wedge \cdots \wedge \alpha_{n}(\underline{a}, \underline{x})\right)$, where $\underline{a}$ are parameters from $\mathcal{M}$ and the $\alpha_{i}(\underline{y}, \underline{x})$ are literals, that is satisfied in some $\mathcal{N} \supseteq \mathcal{M}$ with $\mathcal{N}=T$ is satisfied in $\mathcal{M}$ itself.

The following lemma is taken from $[18,11]$ :

LEMma 8.1. If $T$ is universal, then every model $\mathcal{M}$ of $T$ embeds into an existentially closed (and thus also positive-existentially closed and positive-universally closed) model of $T$.

Proof. Take a well-order $\left\{\phi_{i}\right\}_{i<\alpha}$ of the existential sentences with parameters from $\mathcal{M}$. Define a first chain $\left\{\mathcal{M}_{i}\right\}_{i<\alpha}$ of models of $T$, by letting $\mathcal{M}_{i}$ to be an extension of $\bigcup_{j<i} \mathcal{M}_{j}$ in which $\phi_{i}$ is true (if this extension does not exists, $\mathcal{M}_{i}$ is just $\left.\bigcup_{j<i} \mathcal{M}_{j}\right)$. Now let $\mathcal{M}^{1}$ be $\bigcup_{j<\alpha} \mathcal{M}_{j}$; repeating the construction, ${ }^{20}$ we produce a countable chain $\mathcal{M} \subseteq \mathcal{M}^{1} \subseteq \mathcal{M}^{2} \subseteq \ldots$. The union of this chain is the desired existentially closed extension of $\mathcal{M}$ (notice that this argument works because $T$ is preserved under union of chains, being universal).

Proposition 8.2. Suppose that T has a (positive-existential, positive-universal) model completion $T^{*}$. Then the models of $T^{*}$ are precisely those models of $T$ that are existentially (positive-existentially, positive-universally) closed.

Proof. We give the proof just for the case of the positive-existential model completion $T^{*}$. (The proof for the case of the positive-universal model completion is analogous, and the one for the model completion can be found in [11].)

First, assume that $\mathcal{M} \models T^{*}$. We must show that $\mathcal{M}$ is a positive-existentially closed model of $T$. Since $T \subseteq T^{*}$ by the definition of a positive-existential model completion, $\mathcal{M} \models T$. To show that $\mathcal{M}$ is positive-existentially closed, assume that $\mathcal{N} \supseteq \mathcal{M}$ is an extension of $\mathcal{M}$ that is also a model of $T$, and that $\mathcal{N} \models \phi(\underline{a})$, where $\phi(\underline{a})$ is of the form $\exists \underline{x} .\left(\alpha_{1}(\underline{a}, \underline{x}) \wedge \cdots \wedge \alpha_{n}(\underline{a}, \underline{x})\right)$ for parameters $\underline{a}$ from $\mathcal{M}$ and atoms $\alpha_{i}(y, \underline{x})$. We must show that $\phi(\underline{a})$ is also true in $\mathcal{M}$. Obviously, $\phi$ is a geometric formula, and thus Definition 3.2 and Lemma 3.3 imply that there is the corresponding geometric open formula $\phi^{*}$ with $T=\phi \rightarrow \phi^{*}$ and $T^{*} \models \phi^{*} \rightarrow \phi$. Consequently, we have $\mathcal{N} \models \phi^{*}(\underline{a})$ and also $\mathcal{M} \models \phi^{*}(\underline{a})$ (because $\mathcal{N} \supseteq \mathcal{M}$ and $\phi^{*}$ is open). Since $\mathcal{M}$ is a model of $T^{*}$, this implies that $\mathcal{M} \models \phi(\underline{a})$.

Conversely, suppose that $\mathcal{M}$ is a positive-existentially closed model of $T$. We must show that it is also a model of the geometric theory $T^{*}$. Let $\phi(\underline{a})$ be a geometric sentence with parameters in $\mathcal{M}$ such that $\mathcal{M} \models \phi^{*}(\underline{a})$, where $\phi^{*}$ is the geometric open formula corresponding to $\phi$. By 2. of Definition 3.2, $\mathcal{M}$ can be embedded into a model $\mathcal{N}$ of $T^{*}$. Since $\phi^{*}$ is open, $\mathcal{M}=\phi^{*}(\underline{a})$ implies $\mathcal{N}=$ $\phi^{*}(\underline{a})$, and thus $T^{*} \models \phi^{*} \rightarrow \phi$ yields $\mathcal{N} \models \phi(\underline{a})$. Since the geometric formula $\phi(\underline{a})$ is obviously equivalent to a disjunction of existentially quantified conjunctions of atoms, the fact that $\mathcal{M}$ is positive-existentially closed yields $\mathcal{M}=\phi(\underline{a})$. Thus $\mathcal{M} \models \phi \leftrightarrow \phi^{*}$ holds for all geometric formulae $\phi$ (the implication $\phi \rightarrow \phi^{*}$ being already a logical consequence of $T$ ). It is now easy to show that $\mathcal{M} \models T^{*}$. Let $\phi_{1} \rightarrow \phi_{2}$ be a geometric sequent in the axiomatization of $T^{*}$. We have that $\mathcal{M} \models \phi_{1} \rightarrow \phi_{2}$ iff $\mathcal{M} \models \phi_{1}^{*} \rightarrow \phi_{2}^{*}$, and $T^{*} \models \phi_{1} \rightarrow \phi_{2}$ implies $T^{*} \models \phi_{1}^{*} \rightarrow \phi_{2}^{*}$.

\footnotetext{
${ }^{20}$ The construction needs to be repeated, in order to take care of existential formulae with parameters from $\left|\mathcal{M}^{1}\right| \backslash|\mathcal{M}|$.
} 
Since $T$ and $T^{*}$ agree on open formulae (see 1. and 2. of Definition 3.2 and Lemma 3.1), $T^{*} \models \phi_{1}^{*} \rightarrow \phi_{2}^{*}$ thus yields $T \models \phi_{1}^{*} \rightarrow \phi_{2}^{*}$. Hence, $\mathcal{M} \models T$ implies $\mathcal{M} \models \phi_{1}^{*} \rightarrow \phi_{2}^{*}$, and this in turn implies $\mathcal{M} \models \phi_{1} \rightarrow \phi_{2}$. Thus, we have shown that $\mathcal{M}$ satisfies every geometric sequent in the axiomatization of $T^{*}$, i.e., $\mathcal{M} \equiv T^{*}$.

Notice that Proposition 8.2 implies that $T^{*}$, when it exists, is unique. Clearly, not all universal theories $T$ have a positive-existential model completion or a model completion: there is no general guarantee, for instance, that the class of positive-existentially (existentially) closed models of $T$ is elementary (i.e., that it is the class of models of some first order theory). In the next two subsections we consider conditions for the existence of model completions and positiveexistential model completions, respectively.

8.1. Model completions. A classical result from model theory says that a universal theory $T$ has a model completion iff $T$ has the amalgamation property and the class of the existentially closed models of $T$ is an elementary class (see, e.g., [11]).

We say that a theory $T$ has the amalgamation property ( $A P$ for short) iff for every triple $\mathcal{M}, \mathcal{N}_{1}, \mathcal{N}_{2}$ of models of $T$, for every pair of embeddings $\mu_{1}: \mathcal{M} \rightarrow$ $\mathcal{N}_{1}$ and $\mu_{2}: \mathcal{M} \rightarrow \mathcal{N}_{2}$, there are a further model $\mathcal{N}$ of $T$, and embeddings $\nu_{1}: \mathcal{N}_{1} \rightarrow \mathcal{N}$ and $\nu_{2}: \mathcal{N}_{2} \rightarrow \mathcal{N}$ such that the square

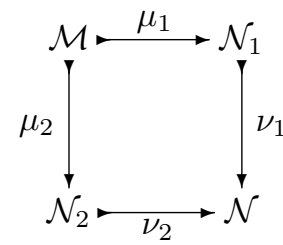

commutes, i.e., $\nu_{1} \circ \mu_{1}=\nu_{2} \circ \mu_{2}$.

THEOREM 8.3. The universal theory $T$ has a model completion iff it has AP and the class of existentially closed models of $T$ is elementary.

Though not explicitly formulated as a result there, this theorem easily follows from the results in [11]. In addition, due to the connection between model completions and positive-existential model completions shown in Proposition 6.3, and the obvious connection between $A P$ and and the injection transfer property $I T$ introduced in the next subsection, it is also a consequence of Theorem 8.6 below.

We conclude this subsection with a proof of the fact that the definition of a model completion given in Definition 6.1 above agrees with the standard definition used in most textbooks and also in $[16,4,5] .{ }^{21}$ Though this result is known, ${ }^{22}$ we include its proof for the sake of completeness.

\footnotetext{
${ }^{21}$ The alternative definition suggested by Proposition 8.4 is in general preferable because it conveniently applies also to theories which might not be universal. We adopted Definition 6.1 in the present article since it makes the connection between model completion and positiveexistential model completion (see Definition 3.2) clearer.

${ }^{22}$ It follows from the information reported in textbooks on model theory, though we could not find an explicit statement of the result in a standard textbook; explicit proofs can, e.g., be found in Appendix B of [15] and in [17].
} 
We say that a theory $T^{*}$ admits quantifier elimination if it satisfies 3 . of Definition 6.1 , i.e., for every formula $\phi(\underline{x})$ there is an open formula $\phi^{*}(\underline{x})$ such that $T^{*} \models \phi \leftrightarrow \phi^{*}$.

Proposition 8.4. Let $T$ be a universal $\Omega$-theory and let $T^{*}$ be a further $\Omega$ theory extending $T$. We have that $T^{*}$ is a model completion of $T$ iff the following two conditions are satisfied:

1. every model of $T$ embeds into a model of $T^{*}$;

2. for every $\Omega$-structure $\mathcal{A}$ that is a model of $T$, we have that $T^{*} \cup \Delta(\mathcal{A})$ is a complete $\Omega^{|\mathcal{A}|}$-theory, i.e., for every $\Omega^{|\mathcal{A}|}$-sentence $\phi$, either $\phi$ or its negation follows from $T^{*} \cup \Delta(\mathcal{A})$.

Proof. First, assume that $T^{*}$ is a model completion of $T$. It is enough to show that, for every model $\mathcal{A}$ of $T$, the theory $T^{*} \cup \Delta(\mathcal{A})$ is complete. Thus, let $\phi(\underline{a})$ be an $\Omega^{|\mathcal{A}|}$-sentence. Since $T^{*}$ admits quantifier elimination, there is an open formula $\phi^{*}$ such that $T^{*} \models \phi \leftrightarrow \phi^{*}$. We have either $\mathcal{A} \models \phi^{*}(\underline{a})$ or $\mathcal{A} \models \neg \phi^{*}(\underline{a})$. We show that in the former case $T^{*} \cup \Delta(\mathcal{A})=\phi(\underline{a})$ and in the latter $T^{*} \cup \Delta(\mathcal{A}) \models \neg \phi(\underline{a})$. We restrict the attention to the former case since the latter can be treated analogously. Thus, assume that $\mathcal{A} \models \phi^{*}(\underline{a})$, and let $\mathcal{M}$ be a model of $T^{*} \cup \Delta(\mathcal{A})$. We must show that $\mathcal{M}=\phi(\underline{a})$. By Robinson's diagram theorem, we can assume without loss of generality that $\mathcal{M}$ is a superstructure of $\mathcal{A}$. Since $\phi^{*}(\underline{a})$ is open, $\mathcal{A} \models \phi^{*}(\underline{a})$ thus implies $\mathcal{M} \models \phi^{*}(\underline{a})$, and since $\mathcal{M}$ is a model of $T^{*}$, this in turn implies $\mathcal{M} \models \phi(\underline{a})$.

To prove the other direction, suppose that $T^{*} \cup \Delta(\mathcal{A})$ is a complete $\Sigma^{|\mathcal{A}|}$-theory for every model $\mathcal{A}$ of $T^{*}$. We must show that $T^{*}$ admits quantifier elimination. Thus, let $\phi(\underline{x})$ be an arbitrary formula. For new constants $\underline{a}$ consider the set of sentences

$$
\Theta:=T^{*} \cup\{\phi(\underline{a})\} \cup\left\{\neg \psi(\underline{a}) \mid \psi \text { is open and } T^{*}=\psi(\underline{a}) \rightarrow \phi(\underline{a})\right\} .
$$

If $\Theta$ is inconsistent, then we have $T^{*} \models \phi(\underline{a}) \rightarrow \psi_{1}(\underline{a}) \vee \cdots \vee \psi_{n}(\underline{a})$ for open formulae $\psi_{1}, \ldots, \psi_{n}$ implying $\phi$. Consequently, we have

$$
T^{*} \models \phi(\underline{x}) \leftrightarrow \psi_{1}(\underline{x}) \vee \cdots \vee \psi_{n}(\underline{x}),
$$

which shows that $T^{*}$ admits quantifier elimination.

Thus, it suffices to show that $\Theta$ cannot be consistent. Suppose to the contrary that $\Theta$ has a model, say $\mathcal{M}$, and let $\mathcal{A}$ be the substructure of $\mathcal{M}$ generated by the $\underline{a}$. Thus, $\mathcal{M}$ is a model of $T^{*} \cup \Delta(\mathcal{A})$ (by Robinson's diagram theorem) that satisfies $\phi(\underline{a})$. Since $T^{*} \cup \Delta(\mathcal{A})$ is complete, this implies

$$
T^{*} \cup \Delta(\mathcal{A}) \models \phi(\underline{a}) .
$$

By compactness, this means that, for some quantifier-free sentence $\psi(\underline{a})$ true in $\mathcal{A}$, we have that $T^{*} \models \psi(\underline{a}) \rightarrow \phi(\underline{a})$. According to the definition of $\Theta, \neg \psi(\underline{a})$ is true in $\mathcal{M}$, and thus also in its substructure $\mathcal{A}$ (because it is quantifier-free), which yields the desired contradiction.

8.2. Positive-existential model completions. We are looking for a result analogous to Theorem 8.3, but for the case of positive-existential model completions. To this aim, we need to identify the semantic properties playing the rôle of amalgamation in this context. 
We say that a theory $T$ has the injection-transfer property (IT for short) iff for every triple $\mathcal{M}, \mathcal{N}_{1}, \mathcal{N}_{2}$ of models of $T$, for every homomorphism $\mu: \mathcal{M} \rightarrow \mathcal{N}_{2}$ and for every embedding $\iota: \mathcal{M} \rightarrow \mathcal{N}_{1}$, there are a further model $\mathcal{N}$ of $T$, an embedding $\iota^{\prime}: \mathcal{N}_{2} \rightarrow \mathcal{N}$ and a homomorphism $\mu^{\prime}: \mathcal{N}_{1} \rightarrow \mathcal{N}$ such that the square

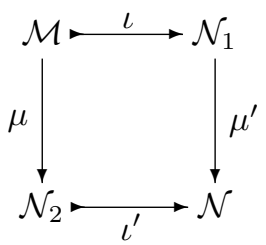

commutes.

Proposition 8.5. If the universal $\Omega$-theory $T$ has a positive-existential model completion $T^{*}$, then $T$ has IT.

Proof. Let $\mu: \mathcal{M} \rightarrow \mathcal{N}_{2}$ be a homomorphism and let $\iota: \mathcal{M} \rightarrow \mathcal{N}_{1}$ be an embedding, where $\mathcal{M}, \mathcal{N}_{1}, \mathcal{N}_{2}$ are models of $T$. By 2. of Definition 3.2, we can freely suppose that $\mathcal{N}_{2}$ is a model of $T^{*}$. By Robinson's diagram theorem, it is sufficient to show the consistency of $T \cup \Delta^{+}\left(\mathcal{N}_{1}\right) \cup \Delta\left(\mathcal{N}_{2}\right)$. Suppose this set is not consistent. By compactness, there are $\theta_{1}\left(\underline{m}, \underline{n}_{1}\right), \theta_{2}\left(\underline{m}, \underline{n}_{2}\right)$ such that $T \cup\left\{\theta_{1}\left(\underline{m}, \underline{n}_{1}\right), \theta_{2}\left(\underline{m}, \underline{n}_{2}\right)\right\}$ is inconsistent, where

- $\underline{m}$ are parameters from $\mathcal{M}$;

- $\underline{n}_{1}, \underline{n}_{2}$ are parameters from $\mathcal{N}_{1}, \mathcal{N}_{2}$ (not belonging to the image of $\iota, \mu$, respectively);

- $\theta_{1}\left(\underline{m}, \underline{n}_{1}\right)$ is a conjunction of ground atoms true in $\mathcal{N}_{1}$;

- $\theta_{2}\left(\underline{m}, \underline{n}_{2}\right)$ is a conjunction of ground literals true in $\mathcal{N}_{2}$.

Let $\phi(\underline{m})$ be $\exists y \cdot \theta_{1}(\underline{m}, y)$, and let $\phi^{*}$ be a geometric open formula such that $T^{*}=$ $\phi \leftrightarrow \phi^{*}$. We have $\mathcal{N}_{1} \models \phi^{*}(\underline{m})$ since $\phi \rightarrow \phi^{*}$ is already a logical consequence of $T$ (see Lemma 3.1). Since $\phi^{*}(\underline{m})$ is geometric and open, and thus preserved under sub- and superstructures, we obtain that it is also true in $\mathcal{M}$ and in $\mathcal{N}_{2}$. Since the latter is a model of $T^{*}$, this implies $\mathcal{N}_{2}=\phi(\underline{m})$. Consequently, $\mathcal{N}_{2}$ is a model of $T \cup\left\{\phi(\underline{m}), \theta_{2}\left(\underline{m}, \underline{n}_{2}\right)\right\}$. Since the parameters $\underline{n}_{1}$ have no fixed meaning in $\mathcal{N}_{2}$, this implies that $T \cup\left\{\theta_{1}\left(\underline{m}, \underline{n}_{1}\right), \theta_{2}\left(\underline{m}, \underline{n}_{2}\right)\right\}$ is satisfiable in $\mathcal{N}_{2}$, which contradicts our assumption that this set is inconsistent.

Propositions 8.2 and 8.5 can be inverted, in the following sense:

THEOREM 8.6. The universal theory $T$ has a positive-existential model completion iff it has IT and the class of positive-existentially closed models of $T$ is elementary.

Proof. The direction from left to right is covered by Proposition 8.2 and Proposition 8.5.

Suppose now that $T$ has $I T$ and that there is a first-order theory $T^{\prime}$ (in principle, not necessarily a geometric one) such that the models of $T^{\prime}$ are exactly the positive-existentially closed models of $T$. Let $\phi(\underline{x})$ be a geometric formula and let $\underline{a}$ be free constants. Define $\Gamma$ as the set of geometric, open, and ground formulae in the signature $\Omega \underline{a}$ (where $\Omega$ is the signature of $T$ ) that are logical consequences of $T^{\prime} \cup\{\phi(\underline{a})\}$. 
We first claim that $\Gamma \cup T^{\prime} \models \phi(\underline{a})$. To show this, consider an arbitrary model $\mathcal{M}$ of $\Gamma \cup T^{\prime}$. Let $\Delta^{-}(\underline{a})$ be the set of negative ground $\Omega^{-}$-literals that are true in $\mathcal{M}$. The set $T^{\prime} \cup \Delta^{-}(\underline{a}) \cup\{\phi(\underline{a})\}$ is consistent. In fact, otherwise compactness yields finitely many elements $\neg \alpha_{1}, \ldots, \neg \alpha_{n}$ of $\Delta^{-}(\underline{a})$ such that $T^{\prime} \cup\left\{\neg \alpha_{1}, \ldots, \neg \alpha_{n}\right\} \cup$ $\{\phi(\underline{a})\}$ is inconsistent, i.e., $T^{\prime} \cup\{\phi(\underline{a})\} \models \alpha_{1} \vee \ldots \vee \alpha_{n}$. Since $\alpha_{1} \vee \ldots \vee \alpha_{n}$ is geometric, open, and ground, it belongs to $\Gamma$, and thus $\mathcal{M} \models \alpha_{1} \vee \ldots \vee \alpha_{n}$, which contradicts the fact that $\neg \alpha_{1}, \ldots, \neg \alpha_{n}$ are true in $\mathcal{M}$. Hence, the set $T^{\prime} \cup \Delta^{-}(\underline{a}) \cup\{\phi(\underline{a})\}$ has a model, say $\mathcal{N}$. Let $\mathcal{A}$ be the substructure of $\mathcal{N}$ generated by the $\underline{a}$. Notice that $\mathcal{A}$ is a model of $T \subseteq T^{\prime}$ because $T$ is universal. In addition, there is a homomorphism from $\mathcal{A}$ into $\mathcal{M}$ since $\mathcal{M}$ satisfies the positive diagram of $\mathcal{A}$. In fact, assume that $\alpha(\underline{a})$ is an atom in the positive diagram of $\mathcal{A}$, but $\mathcal{M} \vDash \alpha(\underline{a})$. Then $\mathcal{M} \models \neg \alpha(\underline{a})$, and thus $\neg \alpha(\underline{a}) \in \Delta^{-}(\underline{a})$, which implies that $\mathcal{N} \models \neg \alpha(\underline{a})$. Since $\neg \alpha(\underline{a})$ is open, this yields $\mathcal{A} \models \neg \alpha(\underline{a})$, which contradicts our assumption that $\alpha(\underline{a})$ belongs to the positive diagram of $\mathcal{A}$. By applying $I T$, we obtain the following commutative square:

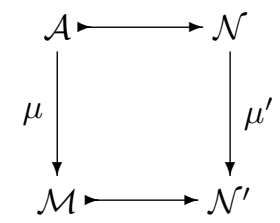

From $\mathcal{N} \models \phi(\underline{a})$, we get $\mathcal{N}^{\prime} \models \phi(\underline{a})$ (since $\phi$ is geometric), and finally $\mathcal{M} \models$ $\phi(\underline{a})$ because $\mathcal{M}$ is positive-existentially closed (since it is a model of $T^{\prime}$ ). This completes the proof of the claim that $\Gamma \cup T^{\prime} \models \phi(\underline{a})$.

From the claim and compactness, we obtain that, for every geometric formula $\phi$, there is a geometric open formula $\phi^{*}$ such that

$$
T^{\prime} \models \phi \rightarrow \phi^{*} \quad \text { and } \quad T^{\prime} \models \phi^{*} \rightarrow \phi .
$$

Let $T^{*}$ be the extension of $T$ axiomatized by the universal closure of the geometric sequents $\phi \rightarrow \phi^{*}$ and $\phi^{*} \rightarrow \phi$. Thus, we have $T \subseteq T^{*} \subseteq T^{\prime}$ and $T^{*}$ is a geometric theory. As every model of $T$ embeds into a model of $T^{\prime}$ by Lemma 8.1, condition 2. of Definition 3.2 is satisfied for $T^{*}$. Since condition 3. of Definition 3.2 comes directly from the construction, $T^{*}$ is a positive-existential model completion of $T$.

8.3. Positive-universal model completions. In this case, we need a property that is, in a sense, dual to IT. Note that IT says that a partial homomorphism (i.e., one from a substructure $\mathcal{M}$ of $\mathcal{N}_{1}$ to $\mathcal{N}_{2}$ ) can be extended in the co-domain (by extending $\mathcal{N}_{2}$ to $\mathcal{N}$ ) so that it becomes totally defined in the domain (i.e, in $\mathcal{N}_{1}$ ). Now we require that a partial homomorphism can be extended in the domain so that it becomes totally "defined" (namely surjective) in the co-domain. In order to formalize this intuition, let us introduce partial homomorphisms formally.

A partial homomorphism from $\mathcal{A}$ to $\mathcal{B}$ is a pair $(m, h)$ given by an embedding $m: \mathcal{A}^{\prime} \rightarrow \mathcal{A}$ and a homomorphism $h: \mathcal{A}^{\prime} \rightarrow \mathcal{B}$ (usually, $m$ is an inclusion, so that a partial homomorphism is a homomorphism from a substructure of $\mathcal{A}$ into $\mathcal{B})$. If such a partial homomorphism is given, $\mathcal{A}^{\prime}$ is said to be the domain of $(m, h)$ and $\mathcal{B}$ the co-domain of $(m, h)$. A partial homomorphism $(m, h)$ is total 
iff $m$ is the identity map. We say that $(m, h)$ is a partial homomorphism among models of $T$ if the structures $\mathcal{A}, \mathcal{B}, \mathcal{A}^{\prime}$ are models of $T$.

An extension of a partial homomorphism $(m, h)$ to the partial homomorphism $(\tilde{m}, \tilde{h})$ is a triple $\iota_{1}, \iota_{2}, \iota_{3}$ of embeddings such that the two squares in the diagram on the left-hand side of Fig. 1 commute. In case $\iota_{1}$ is the identity map, $(\tilde{m}, \tilde{h})$ is said to be a co-domain extension of $(m, h)$ and in case $\iota_{3}$ is the identity map, $(\tilde{m}, \tilde{h})$ is said to be a domain extension of $(m, h)$.
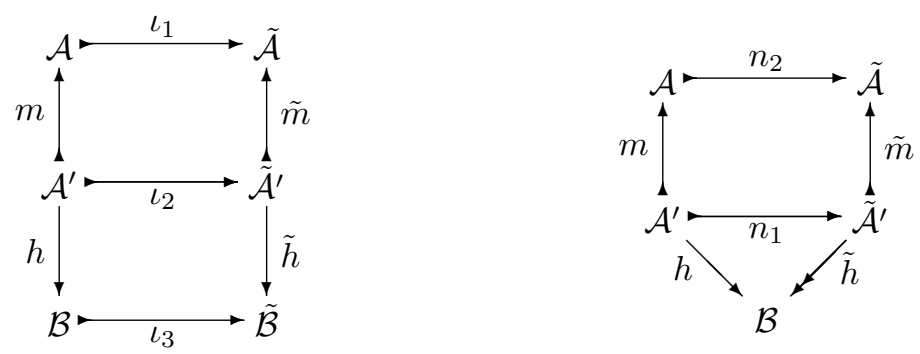

FiguRE 1. Extensions of partial homomorphisms and the $S L$ property.

We say that the universal $\Omega$-theory $T$ has the surjection lifting property $(S L$ for short) iff any partial homomorphism among models of $T$ can be extended in the domain so that it becomes surjective in the co-domain, i.e., the property $S L$ means that, for every partial homomorphism $(m, h)$ among models of $T$, there are embeddings $\tilde{m}, n_{1}, n_{2}$ into models of $T$ and a surjective homomorphism $\tilde{h}$ such that the diagrams of models of $T$ and $\Omega$-homomorphisms shown on the right-hand side of Fig. 1 commute.

Using this definition, we can prove results for the positive-universal case that are analogous to the ones for the positive-existential case.

Proposition 8.7. If the universal $\Omega$-theory $T$ has a positive-universal model completion $T^{*}$, then $T$ has $S L$.

Proof. Let $(m, h)$ be a partial homomorphism with $m: \mathcal{A}^{\prime} \rightarrow \mathcal{A}$ and $h$ : $\mathcal{A}^{\prime} \rightarrow \mathcal{B}$, where we assume without loss of generality that $m$ is a substructure inclusion mapping. Given the statement we want to prove, we can freely suppose that $\mathcal{A}$ is a model of $T^{*}$. Define $\Delta^{-}(\mathcal{B})$ to consist of the negative ground literals $\neg \alpha\left(\underline{a}^{\prime}, \underline{b}\right)$ satisfying the following three conditions:

- $\underline{a}^{\prime}$ are parameters from $\left|\mathcal{A}^{\prime}\right|$,

- $\underline{b}$ are parameters from $|\mathcal{B}| \backslash h\left(\left|\mathcal{A}^{\prime}\right|\right)$,

- $\alpha\left(h\left(\underline{a}^{\prime}\right), \underline{b}\right)$ is false in $\mathcal{B}$.

It is sufficient to show that

$$
\Theta:=T \cup \Delta(\mathcal{A}) \cup \Delta^{-}(\mathcal{B})
$$

is consistent. In fact, if this set of $\left(\Omega \cup|\mathcal{A}| \cup\left(|\mathcal{B}| \backslash h\left(\left|\mathcal{A}^{\prime}\right|\right)\right)\right)$-sentences is consistent, we can easily get the commutative diagrams shown on the right-hand side of Fig. 1 by taking $\tilde{\mathcal{A}}$ to be a model of $\Theta$, and $\tilde{\mathcal{A}}^{\prime}$ to be the $\Omega$-substructure of $\tilde{\mathcal{A}}$ 
generated by $\left|\mathcal{A}^{\prime}\right| \cup\left(|\mathcal{B}| \backslash h\left(\left|\mathcal{A}^{\prime}\right|\right)\right.$. By Robinson's diagram theorem, $\mathcal{A}$ can be embedded into $\tilde{\mathcal{A}}$, which yields the embedding $n_{2}$. In addition, $\tilde{\mathcal{A}}^{\prime}$ is defined as a substructure of $\tilde{\mathcal{A}}$, which yields the embedding $\tilde{m}$. Since $\tilde{\mathcal{A}}$ satisfies the diagram of $\mathcal{A}$, it also satisfies the diagram of the substructure $\mathcal{A}^{\prime}$ of $\mathcal{A}$, and since $\tilde{\mathcal{A}}^{\prime}$ has the elements of $\left|\mathcal{A}^{\prime}\right|$ as generators, it satisfies this diagram as well. This yields the embedding $n_{1}$ from $\mathcal{A}^{\prime}$ into $\tilde{\mathcal{A}}^{\prime}$. To define $\tilde{h}$, we define the images of the generators of $\tilde{\mathcal{A}}^{\prime}$ as follows: the elements $a^{\prime}$ of $\left|\mathcal{A}^{\prime}\right|$ are mapped onto $h\left(a^{\prime}\right)$, and the elements $b$ of $|\mathcal{B}| \backslash h\left(\left|\mathcal{A}^{\prime}\right|\right)$ onto $b$. Obviously, already this mapping of the generators into $|\mathcal{B}|$ is surjective. To see that this mapping can be extended to a well-defined homomorphism from $\tilde{\mathcal{A}}^{\prime}$ to $\mathcal{B}$, it is enough to show that, for any atom $\alpha\left(\underline{a}^{\prime}, \underline{b}\right)$ (where $\underline{a}^{\prime}$ are parameters from $\left|\mathcal{A}^{\prime}\right|$ and $\underline{b}$ are parameters from $\left.|\mathcal{B}| \backslash h\left(\left|\mathcal{A}^{\prime}\right|\right)\right)$ that is true in $\tilde{\mathcal{A}}^{\prime}$, the atom $\alpha\left(h\left(\underline{a}^{\prime}\right), \underline{b}\right)$ is true in $\mathcal{B}$. This is an immediate consequence of the fact that $\tilde{\mathcal{A}}^{\prime}$ satisfies $\Delta^{-}(\mathcal{B})$.

It remains to show that $\Theta$ is consistent. Thus, assume that $\Theta$ is not consistent. Then there are open geometric sentences $\psi\left(\underline{a}^{\prime}, \underline{a}\right), \phi\left(\underline{a}^{\prime}, \underline{b}\right)$, where $\underline{a}^{\prime}$ are parameters from $\left|\mathcal{A}^{\prime}\right|$, $\underline{a}$ are parameters from $|\mathcal{A}| \backslash\left|\mathcal{A}^{\prime}\right|$, and $\underline{b}$ are parameters from $|\mathcal{B}| \backslash h\left(\left|\mathcal{A}^{\prime}\right|\right)$ such that

- $\psi\left(\underline{a}^{\prime}, \underline{a}\right)$ is true in $\mathcal{A}$,

- $\phi\left(h\left(\underline{a}^{\prime}\right), \underline{b}\right)$ is false in $\mathcal{B}$; and

- $T \cup\left\{\psi\left(\underline{a}^{\prime}, \underline{a}\right)\right\} \models \phi\left(\underline{a}^{\prime}, \underline{b}\right)$.

Since $\mathcal{A}$ is a model of $T \cup\left\{\psi\left(\underline{a}^{\prime}, \underline{a}\right)\right\}$, we obtain $\mathcal{A}=\phi\left(\underline{a}^{\prime}, \underline{b}\right)$, and since the components of $\underline{b}$ are free parameters for $\mathcal{A}$, this yields $\mathcal{A} \models \forall \underline{y} . \phi\left(\underline{a}^{\prime}, \underline{y}\right)$. Since $T^{*}$ is the positive-universal model completion of $T$, there is an open co-geometric (and thus geometric) formula $\phi^{*}$ such that $T^{*} \models \phi^{*}(\underline{x}) \leftrightarrow \forall y . \phi(\underline{x}, y)$. Since $\mathcal{A}$ is a model of $T^{*}$, we can infer that $\phi^{*}\left(\underline{a}^{\prime}\right)$ is true in $\mathcal{A}$, and thus also in its substructure $\mathcal{A}^{\prime}$ (since $\phi^{*}$ is open). Since $\phi^{*}$ is geometric, it is preserved under homomorphisms, and thus $\phi^{*}\left(h\left(\underline{a}^{\prime}\right)\right)$ is true in $\mathcal{B}$. This is contradictory to the following three facts: (i) $T \models \phi^{*}(\underline{x}) \rightarrow \phi(\underline{x}, \underline{y})$ (which holds by the dual version of Lemma 3.3); (ii) $\mathcal{B} \not \models \phi\left(h\left(\underline{a}^{\prime}\right), \underline{b}\right)$; and (iii) $\mathcal{B} \models T$.

THEOREM 8.8. The universal theory $T$ has a positive-universal model completion iff it has SL and the class of positive-universally closed models of $T$ is elementary.

Proof. The direction from left to right is covered by Proposition 8.2 and Proposition 8.7.

Suppose that $T$ has $S L$ and that the class of positive-universally closed models of $T$ is elementary, i.e., it is the class of models of a certain first-order theory $T^{\prime} \supseteq T$. Let $\phi(\underline{x}, \underline{y})$ be a given open geometric (and thus also co-geometric) formula, take free constants $\underline{a}$, and let

$$
\Gamma:=\left\{\neg \psi(\underline{a}) \mid \psi(\underline{a}) \text { geometric and open s.t. } T^{\prime} \models \psi(\underline{a}) \rightarrow \forall \underline{y} . \phi(\underline{a}, \underline{y})\right\} .
$$

Following a strategy similar to the one employed in the proof of Theorem 8.6, we claim that $T^{\prime} \cup \Gamma \models \exists y . \neg \phi(\underline{a}, \underline{y})$. To show this, consider an arbitrary model $\mathcal{A}$ of $T^{\prime} \cup \Gamma$. We must show that $\overline{\mathcal{A}}=\exists y . \neg \phi(\underline{a}, y)$. Since, as a model of $T^{\prime}, \mathcal{A}$ is positive-universally closed, it is sufficient to embed $\mathcal{A}$ into some model $\tilde{\mathcal{A}}$ of $T$ such that $\tilde{\mathcal{A}} \models \exists \underline{y} . \neg \phi(\underline{a}, \underline{y})$. Let $\mathcal{A}^{\prime}$ be the substructure of $\mathcal{A}$ generated by $\underline{a}^{\mathcal{A}}$, 
i.e., the interpretation of the components of $\underline{a}$ in $\mathcal{A}$. The set

$$
T^{\prime} \cup\{\exists \underline{y} . \neg \phi(\underline{a}, \underline{y})\} \cup \Delta^{+}\left(\mathcal{A}^{\prime}\right)
$$

is consistent since $\mathcal{A}^{\prime}$ is a model of $\Gamma$, and consequently it has a model $\mathcal{B}$. By Robinson's diagram theorem, there is a homomorphism $h: \mathcal{A}^{\prime} \rightarrow \mathcal{B}$ and by the $S L$ property we can fill the diagram on the right-hand side of Fig. 1, where we can assume without loss of generality that the embeddings $m, \tilde{m}, n_{1}, n_{2}$ in this diagram are in fact inclusions. Since $\mathcal{B} \models \exists y . \neg \phi(\underline{a}, y)$ and since $\tilde{h}$ is surjective, we get $\tilde{\mathcal{A}}^{\prime} \models \exists \underline{y} . \neg \phi(\underline{a}, \underline{y})$, and finally $\tilde{\mathcal{A}} \models \exists \underline{y} . \neg \phi(\underline{a}, \underline{y})$, as desired. This completes the proof of the claim that $T^{\prime} \cup \Gamma \models \exists y \cdot \neg \bar{\phi}(\underline{a}, y)$.

From the claim and compactness it follows that for every co-geometric formula $\forall \underline{y} . \phi(\underline{x}, \underline{y})^{23}$ there exists an open co-geometric formula $\phi^{*}(\underline{x})$ such that

$$
T^{\prime} \models \forall \underline{y} \cdot \phi(\underline{x}, \underline{y}) \leftrightarrow \phi^{*}(\underline{x}) .
$$

Let $T^{*}$ be the extension of $T$ obtained by adding the co-geometric sequents $\forall \underline{y} . \phi(\underline{x}, \underline{y}) \rightarrow \phi^{*}(\underline{x})$ and $\phi^{*}(\underline{x}) \rightarrow \forall \underline{y} \cdot \phi(\underline{x}, \underline{y})$. Then $T \subseteq T^{*} \subseteq T^{\prime}$ and $T^{*}$ is a co-geometric theory. As every model of $T$ embeds into a model of $T^{\prime}$ by Lemma 8.1, condition 2. of Definition 6.6 is satisfied for $T^{*}$. Since condition 3. of Definition 6.6 comes directly from the construction, $T^{*}$ is a positive-universal model completion of $T$.

§9. Alternative proofs. The proofs of our decidability transfer results were all divided into two parts. First, a proposition that characterized satisfiability of a constraint in the combined theory, based on the existence of certain models and homomorphisms, and the satisfiability of certain constraints in the component theories. Second, a non-deterministic algorithm that checks the conditions of the proposition. For this algorithm to be effective, local finiteness of the connecting theory $T_{0}$ and countability of the involved signatures are required. We also used these assumptions in the proofs of the propositions, but already mentioned there that this is done just for the sake of simplicity. Here we give alternative proofs of the relevant propositions from $\S 4, \S 5$, and $\S 6$, relying on the slightly deeper model-theoretic machinery introduced in the previous section. The main feature of these alternative proofs is that they use neither local finiteness of $T_{0}$ nor countability of the involved signatures.

We first need the following extended IT property, which is an interesting consequence of $T_{0}$-positive-existential compatibility:

Proposition 9.1. Let $T_{0} \subseteq T$ be theories over the respective signatures $\Omega_{0} \subseteq$ $\Omega$ such that $T_{0}$ is universal and $T$ is $T_{0}$-positive-existentially compatible. Let $\mathcal{A}, \mathcal{C}$ be $\Omega_{0}$-structures that are models of $T_{0}$, and let $\mathcal{M}$ be an $\Omega$-structure that is a models of $T$. For every $\Omega_{0}$-homomorphism $\mu: \mathcal{A} \rightarrow \mathcal{M}_{\mid \Omega_{0}}$ and for every $\Omega_{0}$-embedding $\iota: \mathcal{A} \rightarrow \mathcal{C}$, there are a further $\Omega$-structure $\mathcal{N}$ that is a model $T$, an $\Omega$-embedding $\iota^{\prime}: \mathcal{M} \rightarrow \mathcal{N}$ and a $\Omega_{0}$-homomorphism $\mu^{\prime}: \mathcal{C} \rightarrow \mathcal{N}_{\mid \Omega_{0}}$ such that the square

\footnotetext{
${ }^{23}$ Note that we can restrict the attention to such co-geometric formulae, i.e. co-geometric formulae in prenex form.
} 


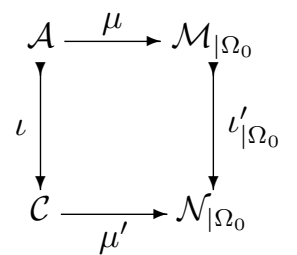

commutes. Moreover, if $\mathcal{M} \models T \cup T_{0}^{*}$, then the embedding $\iota^{\prime}$ can be taken to be elementary.

Proof. Similarly to the proof of Proposition 8.5, we need to show that $T \cup$ $\Delta_{\Omega_{0}}^{+}(\mathcal{C}) \cup \Delta_{\Omega}(\mathcal{M})$ is consistent. Again, if this is not the case, we have that there are formulae $\theta_{1}(\underline{a}, \underline{c}), \theta_{2}(\underline{a}, \underline{m})$ such that $T \cup\left\{\theta_{1}(\underline{a}, \underline{c}), \theta_{2}(\underline{a}, \underline{m})\right\}$ is inconsistent, where

- $\underline{a}$ are parameters from $\mathcal{A}$;

- $\underline{c}, \underline{m}$ are parameters from $\mathcal{C}, \mathcal{M}$ (not belonging to the image of $\iota, \mu$, respectively);

- $\theta_{1}(\underline{a}, \underline{c})$ is a conjunction of ground $\Omega_{0}^{\underline{a}, \underline{c}}$-atoms true in $\mathcal{C}$;

- $\theta_{2}(\underline{a}, \underline{m})$ is a conjunction of ground $\Omega \underline{a}, \underline{m}$-literals true in $\mathcal{M}$.

Let $\phi(\underline{a})$ be $\exists y \cdot \theta_{1}(\underline{a}, y)$, and let $\phi^{*}$ be the corresponding geometric open formula with $T_{0} \models \phi \rightarrow \phi^{*}$ and $T_{0}^{*} \models \phi^{*} \rightarrow \phi$. Then $\mathcal{C} \models \phi(\underline{a})$ implies $\mathcal{C} \models \phi^{*}(\underline{a})$ since $\mathcal{C}$ is a model of $T_{0}$. Since $\phi^{*}(\underline{a})$ is geometric and open, we obtain that this formula is also true in $\mathcal{A}$ and in $\mathcal{M}$. The latter can be embedded into a model $\mathcal{M}_{0}$ of $T \cup T_{0}^{*}$, which also satisfies $\phi^{*}(\underline{a})$. But then $T_{0}^{*} \models \phi^{*} \rightarrow \phi$ implies $\mathcal{M}_{0} \models \phi(\underline{a})$, which is a contradiction since $T \cup\left\{\phi(\underline{a}), \theta_{2}(\underline{a}, \underline{m})\right\}$ was supposed to be inconsistent. (Note that $\mathcal{M}_{0}=\theta_{2}(\underline{a}, \underline{m})$ follows from $\mathcal{M}=\theta_{2}(\underline{a}, \underline{m})$ because $\theta_{2}$ is open.)

In case $\mathcal{M}$ is a model of $T \cup T_{0}^{*}$, we can replace $\Delta_{\Omega}(\mathcal{M})$ by the elementary diagram $\Delta_{\Omega}^{e}(\mathcal{M})$ of $\mathcal{M}$ and get an elementary embedding $\iota^{\prime}$, because there is no need of considering the extension $\mathcal{M}_{0}$ of $\mathcal{M}$.

Let us now give an alternative proof of Proposition 4.2. The only-if direction is shown as in the proof given in $\S 4$. Using the proposition we have just shown, the proof of the more interesting if direction is very simple. From the conditions 1.-5. of Proposition 4.2, we obtain an $\Omega_{0}$-homomorphism $\nu: \mathcal{A} \rightarrow \mathcal{B}$ from an $\Omega_{0}$-substructure $\mathcal{A}$ of a model $\mathcal{N}^{\prime}$ of $T_{1}$ satisfying $\Gamma_{1}$ to an $\Omega_{0}$-substructure $\mathcal{B}$ of a model $\mathcal{N}^{\prime \prime}$ of $T_{2}$ satisfying $\Gamma_{2}$ (see the proof in $\S 4$ ). Proposition 4.2 is proved if we can build an extension of $\nu$ to an $\Omega_{0}$-homomorphism $\mathcal{N}_{\mid \Omega_{0}}^{\prime} \rightarrow \mathcal{N}_{\mid \Omega_{0}}$, where $\mathcal{N}$ is a suitable $\Omega_{2}$-superstructure of $\mathcal{N}^{\prime \prime}$ that is a model of $T_{2}$. (Note that $\mathcal{N}^{\prime \prime}$ then also satisfies $\Gamma_{2}$ since constraints are open formulae.) But such an extension is obtained by an application of Proposition 9.1: take as $\iota$ the inclusion of $\mathcal{A}$ into $\mathcal{N}^{\prime}$, and as $\mu$ the composition of $\nu$ with the inclusion of $\mathcal{B}$ into $\mathcal{N}^{\prime \prime}$.

In the remainder of this section we show that similar arguments give alternative proofs of the other relevant propositions from $\S 4$ and $\S 5$. These proofs are a bit more involved since an iteration of the simple argument from above is needed.

An alternative proof of Proposition $\mathbf{4 . 4}$ can be given as follows. We are given models $\mathcal{N}^{0}, \mathcal{M}^{0}$ of $T_{1}, T_{2}$ respectively, where $\mathcal{N}^{0}$ satisfies the constraint $\Gamma_{1}$ 
and $\mathcal{M}^{0}$ satisfies the constraint $\Gamma_{2} ; \mathcal{N}^{0}$ has $\Omega_{0}$-substructures $\mathcal{A}, \mathcal{A}^{\prime}$, and $\mathcal{M}^{0}$ has $\Omega_{0}$-substructures $\mathcal{B}, \mathcal{B}^{\prime}$. We are also given $\Omega_{0}$-homomorphisms $\nu: \mathcal{A} \rightarrow \mathcal{B}$ and $\mu: \mathcal{B}^{\prime} \rightarrow \mathcal{A}^{\prime}$. We can freely suppose that $\mathcal{N}^{0}, \mathcal{M}^{0}$ are also models of $T_{0}^{*}$, by the positive-existential compatibility assumptions.

The proposition is proved if we succeed in producing elementary extensions $\mathcal{N}^{\infty}, \mathcal{M}^{\infty}$ of $\mathcal{N}, \mathcal{M}$ endowed with $\Omega_{0}$-homomorphisms

$$
\nu^{\infty}: \mathcal{N}_{\mid \Omega_{0}}^{\infty} \rightarrow \mathcal{M}_{\mid \Omega_{0}}^{\infty}, \quad \mu^{\infty}: \mathcal{M}_{\mid \Omega_{0}}^{\infty} \rightarrow \mathcal{N}_{\mid \Omega_{0}}^{\infty}
$$

extending $\nu$ and $\mu$, respectively. To this aim, we define elementary chains of models

$$
\mathcal{N}^{0} \subseteq \mathcal{N}^{1} \subseteq \cdots \quad \text { and } \quad \mathcal{M}^{0} \subseteq \mathcal{M}^{1} \subseteq \cdots
$$

as well as homomorphisms

$$
\nu^{k}: \mathcal{N}_{\mid \Omega_{0}}^{k} \rightarrow \mathcal{M}_{\mid \Omega_{0}}^{k+1}, \quad \mu^{j}: \mathcal{M}_{\mid \Omega_{0}}^{j} \rightarrow \mathcal{N}_{\mid \Omega_{0}}^{j}
$$

$(k \geq 0, j \geq 1)$ such that $\nu \subseteq \nu^{k} \subseteq \nu^{k+1}$ and $\mu \subseteq \mu^{j} \subseteq \mu^{j+1}$. Once these chains are constructed, it is sufficient to take unions in order to get the desired structures and homomorphisms $\mathcal{N}^{\infty}, \mathcal{M}^{\infty}, \nu^{\infty}, \mu^{\infty} .{ }^{24}$ These chains can easily be built by using Proposition 9.1 . For instance, to get $\mathcal{M}_{1}$ and $\nu_{0}$, it is sufficient to fill the square

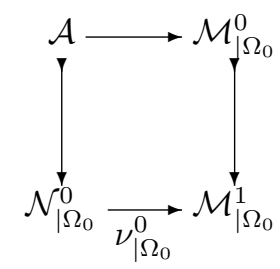

where the top horizontal homomorphism is the composition of $\nu$ with the inclusion $\mathcal{B} \subseteq \mathcal{M}_{\mid \Omega_{0}}^{0}$. Note that we can get an elementary embedding of $\mathcal{M}^{0}$ into $\mathcal{M}^{1}$ since $\mathcal{M}^{0} \models T_{0}^{*} \cup T_{2}$. The fact that the embedding is elementary in turn implies that $\mathcal{M}^{1}$ is also a model of $T_{0}^{*} \cup T_{2}$ (and not just of $T_{2}$ ). Thus, $\mathcal{M}^{1}$ satisfies the same conditions as $\mathcal{M}^{0}$.

To get $\mathcal{N}_{1}$ and $\mu_{1}$, we use Proposition 9.1 to fill the square

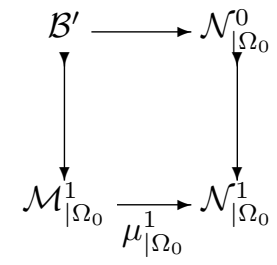

where the top horizontal homomorphism is the composite of $\mu$ with the inclusion $\mathcal{A}^{\prime} \subseteq \mathcal{N}_{\mid \Omega_{0}}^{0}$ and the left vertical morphism is the composite inclusion $\mathcal{B}^{\prime} \subseteq \mathcal{M}_{0} \subseteq$ $\mathcal{M}_{1}$. Again, the embedding of $\mathcal{N}^{0}$ into $\mathcal{N}^{1}$ can be assumed to be elementary since $\mathcal{N}^{0} \models T_{0}^{*} \cup T_{1}$, and this implies that $\mathcal{N}^{1}$ is also a model of $T_{0}^{*} \cup T_{1}$.

For the inductive cases, the same arguments can be applied.

\footnotetext{
${ }^{24}$ Recall the elementary chain theorem [11], according to which the union of an elementary chain of models is elementarily equivalent to each member of the chain.
} 
An alternative proof of Proposition $\mathbf{5 . 3}$ can be given as follows. Here we are given a model $\mathcal{M}^{0}$ of $T$ that satisfies the constraint $\Gamma$, and a pair of $\Omega_{0}$-substructures $\mathcal{A}, \mathcal{B}$ of $\mathcal{M}^{0}$ together with an $\Omega_{0}$-homomorphism $\nu: \mathcal{A} \rightarrow \mathcal{B}$. Again, we can assume without loss of generality that $\mathcal{M}^{0} \models T \cup T_{0}^{*}$.

The proposition is proved, if we succeed in producing an elementary extension $\mathcal{M}^{\infty}$ of $\mathcal{M}^{0}$ endowed with an $\Omega_{0}$-homomorphism

$$
\nu^{\infty}: \mathcal{M}_{\mid \Omega_{0}}^{\infty} \rightarrow \mathcal{M}_{\mid \Omega_{0}}^{\infty}
$$

extending $\nu$. To this aim, we define an elementary chain of models

$$
\mathcal{M}^{0} \subseteq \mathcal{M}^{1} \subseteq \cdots
$$

as well as homomorphisms

$$
\nu^{k}: \mathcal{M}_{\mid \Omega_{0}}^{k} \rightarrow \mathcal{M}_{\mid \Omega_{0}}^{k+1}
$$

( $k \geq 0$ ) such that $\nu \subseteq \nu^{k} \subseteq \nu^{k+1}$ (once this is settled, it is sufficient to take unions in order to get the desired structure $\mathcal{M}^{\infty}$ and homomorphism $\nu^{\infty}$ ). To get $\mathcal{M}_{1}$ and $\nu_{0}$, we use Proposition 9.1 to fill the square

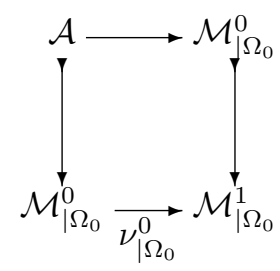

where the top horizontal homomorphism is the composite of $\nu$ with the inclusion $\mathcal{B} \subseteq \mathcal{M}_{\mid \Omega_{0}}^{0}$. As in the previous proof, the embedding of $\mathcal{M}^{0}$ into $\mathcal{M}^{1}$ can be taken to be elementary, and thus $\mathcal{M}^{1}$ is again a model of $T \cup T_{0}^{*}$.

To get inductively $\mathcal{M}_{k+1}$ and $\nu_{k}$, one proceeds in the same way.

In order to give an alternative proof of Proposition 6.9, we first need to extend the $S L$ property, in the same way we have extended the $I T$ property. However, we do not need the extension of $S L$ in its full strength: we can limit ourselves to the case in which the partial homomorphism to be extended is a total one.

Proposition 9.2. Let $T_{0} \subseteq T$ be theories over the respective signatures $\Omega_{0} \subseteq$ $\Omega$ such that $T_{0}$ is universal and $T$ is $T_{0}$-positive universally compatible. Let $\mathcal{A}$ be an $\Omega_{0}$-structure that is a model of $T_{0}$, and let $\mathcal{M}$ be an $\Omega$-structure that is a model of $T$. For every $\Omega_{0}$-homomorphism $\nu: \mathcal{M}_{\mid \Omega_{0}} \rightarrow \mathcal{A}$, there are a further $\Omega$-structure $\mathcal{N}$ that is a model $T$, an $\Omega_{0}$-structure $\mathcal{B}$ that is a model of $T_{0}$, an $\Omega$-embedding $\iota: \mathcal{M} \rightarrow \mathcal{N}, \Omega_{0}$-embeddings $m: \mathcal{M}_{\mid \Omega_{0}} \rightarrow \mathcal{B}, n: \mathcal{B} \rightarrow \mathcal{N}_{\mid \Omega_{0}}$ and a surjective $\Omega_{0}$-homomorphism $\mu: \mathcal{B} \rightarrow \mathcal{A}$ such that the diagram 


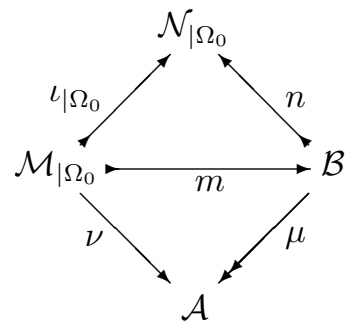

commutes. Moreover, if $\mathcal{M} \models T_{0} \cup T_{0}^{*}$, then $\iota$ can be taken to be elementary.

PROOF. Intuitively, the proposition says that the total $\Omega_{0}$-homomorphism $\nu$ can be extended to a partial surjective $\Omega_{0}$-homomorphism whose domain is an $\Omega_{0}$-substructure of an $\Omega$-superstructure of the domain of $\nu$. Similarly to the approach used in the proof of Proposition 8.7, we define $\Delta_{\Omega_{0}}^{-}(\mathcal{A})$ to consist of the negative ground literals $\neg \alpha(\underline{m}, \underline{a})$ satisfying the following three conditions:

- $\underline{m}$ are parameters from $\left|\mathcal{M}_{\mid \Omega_{0}}\right|$,

- $\underline{a}$ are parameters from $|\mathcal{A}| \backslash \nu\left(\left|\mathcal{M}_{\mid \Omega_{0}}\right|\right)$,

- $\alpha(\nu(\underline{m}), \underline{a})$ is false in $\mathcal{A}$.

It is sufficient to show that

$$
\Theta:=T \cup \Delta_{\Omega}(\mathcal{M}) \cup \Delta_{\Omega_{0}}^{-}(\mathcal{A})
$$

is consistent. In fact, if $\Theta$ is consistent then we can take as $\mathcal{N}$ a model of $\Theta$, and as $\mathcal{B}$ the $\Omega_{0}$-substructure of $\mathcal{N}_{\mid \Omega_{0}}$ generated by $|\mathcal{A}| \cup\left|\mathcal{M}_{\mid \Omega_{0}}\right|$. To show that the required embeddings $m, n$ and the surjective homomorphism $\mu$ exist, we can proceed as in the proof of Proposition 8.7. Note that $\mathcal{B}$ is a model of $T_{0}$ since $\mathcal{N}_{\mid \Omega_{0}}$ is a model of $T_{0} \subseteq T, \mathcal{B}$ is an $\Omega_{0}$-substructure of $\mathcal{N}_{\mid \Omega_{0}}$, and $T_{0}$ is universal.

Thus, assume that $\Theta$ is inconsistent. Then there are open geometric (and co-geometric) formulae $\psi\left(\underline{x}, \underline{x}^{\prime}\right), \phi(\underline{x}, \underline{y})$ (where $\psi$ is over the signature $\Omega$ and $\phi$ is over the signature $\left.\Omega_{0}\right)$ and parameters $\underline{m}$ from $\left|\mathcal{M}_{\mid \Omega_{0}}\right|, \underline{m}^{\prime}$ from $|\mathcal{M}| \backslash\left|\mathcal{M}_{\mid \Omega_{0}}\right|$ and $\underline{a}$ from $|\mathcal{A}| \backslash \nu\left(\left|\mathcal{M}_{\mid \Omega_{0}}\right|\right)$ such that

- $\psi\left(\underline{m}, \underline{m}^{\prime}\right)$ is true in $\mathcal{M}$,

- $\phi(\nu(\underline{m}), \underline{a})$ is false in $\mathcal{A}$; and

- $T \cup\left\{\psi\left(\underline{m}, \underline{m}^{\prime}\right)\right\} \models \phi(\underline{m}, \underline{a})$, which is the same as $T \cup\left\{\psi\left(\underline{m}, \underline{m^{\prime}}\right)\right\} \models \forall \underline{y} . \phi(\underline{m}, \underline{y})$ since the parameters $\underline{a}$ have no fixed meaning in $\mathcal{M}$.

Since there is a positive-universal model completion $T_{0}^{*}$ of $T_{0}$, there is an open cogeometric (and thus geometric) formula $\phi^{*}$ such that $T_{0}^{*} \models \phi^{*}(\underline{x}) \leftrightarrow \forall y . \phi(\underline{x}, y)$. This implies that $\phi^{*}(\underline{m})$ is true in $\mathcal{M}$ : in fact, by $T_{0}$-positive universal compatibility of $T$, we can embed $\mathcal{M}$ into a model $\mathcal{M}^{\prime}$ of $T \cup T_{0}^{*}$, thus obtaining $\mathcal{M}^{\prime} \models \phi^{*}(\underline{m})$ and finally $\mathcal{M} \models \phi^{*}(\underline{m})$ (recall that $\psi\left(\underline{m}, \underline{m^{\prime}}\right), \phi^{*}(\underline{m})$ are both Boolean combinations of ground atoms and hence are preserved under building sub- and superstructures).

Since $\phi^{*}$ is geometric, it is preserved under homomorphisms, and thus $\phi^{*}(\nu(\underline{m}))$ is true in $\mathcal{A}$. This is contradictory to the following three facts: (i) $T_{0} \models \phi^{*}(\underline{x}) \rightarrow$ $\forall \underline{y} . \phi(\underline{x}, \underline{y})$ (which holds by the dual version of Lemma 3.3); (ii) $\mathcal{A} \not \models \phi(\nu(\underline{m}), \underline{a})$; and (iii) $\mathcal{A} \models T_{0}$. 
In case $\mathcal{M} \models T_{0} \cup T_{0}^{*}$, we can argue as above, but we replace $\Delta_{\Omega}(\mathcal{M})$ in the definition of $\Theta$ by the elementary diagram of $\mathcal{M}$ in the signature $\Omega$ (the argument in fact now works without introducing the model $\mathcal{M}^{\prime}$ ).

In the alternative proof of Proposition 6.9, we again restrict the attention to the nontrivial if direction. From the conditions of the proposition, we obtain an $\Omega_{0}$-homomorphism $\nu: \mathcal{A} \rightarrow \mathcal{B}$ from an $\Omega_{0}$-substructure $\mathcal{A}$ of a model $\mathcal{N}^{0}$ of $T_{1}$ satisfying $\Gamma_{1}$ to an $\Omega_{0}$-substructure $\mathcal{B}$ of a model $\mathcal{M}^{0}$ of $T_{2}$ satisfying $\Gamma_{2}$. We may also assume that $\mathcal{N}^{0}\left(\mathcal{M}^{0}\right)$ is a model of the positive-universal (positive-existential) completion of $T_{0}$.

First, we apply Proposition 9.1 to the embedding from $\mathcal{A}$ into $\mathcal{N}^{0}$ and the $\Omega_{0}$-homomorphism from $\mathcal{A}$ to $\mathcal{M}^{0}$ obtained by the composition of $\nu$ with the embedding of $\mathcal{B}$ into $\mathcal{M}^{0}$. This application yields an elementary extension $\mathcal{M}^{1}$ of $\mathcal{M}^{0}$ and an $\Omega_{0}$-homomorphism $\nu_{0}: \mathcal{N}_{\mid \Omega_{0}}^{0} \rightarrow \mathcal{M}_{\mid \Omega_{0}}^{1}$ extending $\nu$. Since $\mathcal{M}^{0}$ is a model of $T_{2}$ and the positive-existential completion of $T_{0}$ that also satisfies $\Gamma_{2}$, the same is true for its elementary extension $\mathcal{M}^{1}$.

Second, we apply Proposition 9.2 to the $\Omega_{0}$-homomorphism $\nu_{0}: \mathcal{N}_{\mid \Omega_{0}}^{0} \rightarrow \mathcal{M}_{\mid \Omega_{0}}^{1}$. This application yields an elementary extension $\mathcal{N}^{1}$ of $\mathcal{N}^{0}$, a model $\mathcal{A}^{1}$ of $T_{0}$ that is an $\Omega_{0}$-superstructure of $\mathcal{N}^{0}$ and an $\Omega_{0}$-substructure of $\mathcal{N}^{1}$, and a surjective $\Omega_{0^{-}}$ homomorphism $\mu_{1}: \mathcal{A}^{1} \rightarrow \mathcal{M}_{\mid \Omega_{0}}^{1}$ such that the commutation properties stated in Proposition 9.2 are satisfied. The fact that $\mathcal{N}^{0}$ is a model of $T_{1}$ and the positive-universal completion of $T_{0}$ that also satisfies $\Gamma_{1}$ implies that the same is true for its elementary extension $\mathcal{N}^{1}$.

Next, we can apply Proposition 9.1 to the embedding from $\mathcal{A}^{1}$ into $\mathcal{N}^{1}$ and the $\Omega_{0}$-homomorphism $\mu_{1}$ from $\mathcal{A}^{1}$ to $\mathcal{M}_{\mid \Omega_{0}}$, then Proposition 9.2 to the homomorphism $\nu_{1}$ obtained this way, etc. Continuing this way, we can construct the following chains:

- an elementary chain of $\Omega_{1}$-structures $\mathcal{N}^{0} \subseteq \mathcal{N}^{1} \subseteq \ldots$;

- an elementary chain of $\Omega_{2}$-structures $\mathcal{M}^{0} \subseteq \mathcal{M}^{1} \subseteq \ldots$;

- a chain of $\Omega_{0}$-structures

$$
\mathcal{N}_{\mid \Omega_{0}}^{0} \subseteq \mathcal{A}^{1} \subseteq \mathcal{N}_{\mid \Omega_{0}}^{1} \subseteq \mathcal{A}^{2} \subseteq \mathcal{N}_{\mid \Omega_{0}}^{2} \subseteq \cdots ;
$$

- a chain of $\Omega_{0}$-homomorphisms (for $k \geq 0$ )

$$
\nu_{k}: \mathcal{N}_{\mid \Omega_{0}}^{k} \rightarrow \mathcal{M}_{\mid \Omega_{0}}^{k+1}
$$

- a chain of surjective $\Omega_{0}$-homomorphisms (for $k \geq 0$ )

$$
\mu_{k+1}: \mathcal{A}^{k+1} \rightarrow \mathcal{M}_{\mid \Omega_{0}}^{k+1},
$$

where the above homomorphisms satisfy the condition

$$
\nu_{0} \subseteq \mu_{1} \subseteq \nu_{1} \subseteq \mu_{2} \subseteq \cdots .
$$

Once these chains are constructed, we obtain elementary extensions $\mathcal{N}^{\infty}, \mathcal{M}^{\infty}$ of $\mathcal{N}^{0}, \mathcal{M}^{1}$ endowed with a surjective $\Omega_{0}$-homomorphism

$$
\nu^{\infty}: \mathcal{N}_{\mid \Omega_{0}}^{\infty} \rightarrow \mathcal{M}_{\mid \Omega_{0}}^{\infty}
$$

extending $\nu_{0}$ by taking the unions over the chains. 
§10. Conclusion. We have introduced a new scheme for combining manysorted theories, and have shown under which conditions decidability of the universal fragment of the component theories transfers to their combination. Though this kind of combination has been considered before in restricted cases $[20,1,33]$, it has not been investigated in the general algebraic setting considered here.

In this article, we mainly concentrated on the simplest case of connecting many-sorted theories where there is just one connection function. The approach was then extended to the case of several independent connection functions, and to variants of the general combination scheme where the connection function must satisfy additional properties or where a theory is connected with itself.

On the one hand, our results are more general than the combination results for $\mathcal{E}$-connections of abstract description systems shown in [20] since they are not restricted to Boolean-based equational theories, which are closely related to abstract description systems (see Example 2.2 and $\S 7$ ). For instance, we have shown in Example 4.5 that any pair of theories $T_{1}, T_{2}$ extending a universal theory $T_{0}$ that is effectively locally finite and Gaussian satisfies the prerequisites of our transfer theorem. Examples of such theories having nothing to do with Boolean-based equational theories can be found in $[4,5]$.

On the other hand, in the $\mathcal{E}$-connection approach introduced in [20], one usually considers not only the modal operator induced by a connecting relation $E$ (see Example 2.2), but also the modal operator induced by its inverse $E^{-1}$. It is not adequate to express these two modal operators by independent connection functions going in different directions since this does not capture the relationships that must hold between them. For example, if $\diamond$ is the diamond operator induced by the connecting relation $E$, and $\square^{-}$is the box operator induced by its inverse $E^{-}$, then the formulae $x \rightarrow \square^{-} \diamond x$ and $\triangleright^{-} y \rightarrow y$ are valid in the $\mathcal{E}$-connection.

In order to express these relationships in the algebraic setting without assuming the presence of the Boolean operators in the shared theory, one can replace the logical implication $\rightarrow$ by a partial order $\leq$, and require that $x \leq r(\ell(x))$ and $\ell(r(y)) \leq y$ hold for the connection functions $r, \ell$ generalizing the diamond and the inverse box operator. If $\ell, r$ are also order preserving, then this means that $\ell, r$ is a pair of adjoint functions for the partial order $\leq$. This suggests an alternative way of connecting theories through pairs of adjoint functions. Again, we can show transfer of decidability provided that certain algebraic conditions are satisfied [2].

The approach in [2] can handle inverse connecting relations, and thus captures (in an algebraic setting) more of the results from [20] than the one presented here. It is, however, also less general than the approach presented here since it requires the additional assumption that the theories to be connected are equipped with a partial order and that the connection functions are adjoint functions for this partial order. Intuitively, this makes the theories that can be handled more similar to Boolean-based equational theories since something like logical entailment is assumed to be present. 
[1] Farid Ajili and Claude Kirchner, A modular framework for the combination of symbolic and built-in constraints, Proceedings of the Fourteenth International Conference on Logic Programming (Leuven, Belgium) (Lee Naish, editor), The MIT press, 1997, pp. 331345.

[2] Franz Baader and Silvio Ghilardi, Connecting many-sorted structures and theories through adjoint functions, Proceedings of the 5th International Workshop on Frontiers of Combining Systems (FroCoS 2005) (Vienna, Austria) (B. Gramlich, editor), Lecture Notes in Artificial Intelligence, vol. 3717, Springer-Verlag, 2005, pp. 31-47.

$[3] \longrightarrow$, Connecting many-sorted theories, Proceedings of the 20th International Conference on Automated Deduction (CADE-05) (Tallinn (Estonia)), Lecture Notes in Artificial Intelligence, vol. 3632, Springer-Verlag, 2005, pp. 278-294.

[4] Franz Baader, Silvio Ghilardi, and Cesare Tinelli, A new combination procedure for the word problem that generalizes fusion decidability results in modal logics, Proceedings of the Second International Joint Conference on Automated Reasoning (IJCAR'04) (Cork, Ireland), Lecture Notes in Artificial Intelligence, vol. 3097, Springer-Verlag, 2004, pp. 183-197.

[5] - A new combination procedure for the word problem that generalizes fusion decidability results in modal logics, Information and Computation, vol. 204 (2006), no. 10, pp. 1413-1452.

[6] Franz Baader, Carsten lutz, Holger Sturm, and Frank Wolter, Fusions of description logics and abstract description systems, Journal of Artificial Intelligence Research), vol. 16 (2002), pp. 1-58.

[7] Franz BaAder and Cesare Tinelli, A new approach for combining decision procedures for the word problem, and its connection to the Nelson-Oppen combination method, Proceedings of the 14th International Conference on Automated Deduction (Townsville, Australia) (William McCune, editor), Lecture Notes in Artificial Intelligence, vol. 1249, SpringerVerlag, 1997, pp. 19-33.

[8] - Deciding the word problem in the union of equational theories, Information and Computation, vol. 178 (2002), no. 2, pp. 346-390.

[9] Patrick Blackburn, Maarten de Rijke, and Yde Venema, Modal logic, Cambridge Tracts in Theoretical Computer Science, vol. 53, Cambridge University Press, 2001.

[10] Maria Paola Bonacina, Silvio Ghilardi, Enrica Nicolini, Silvio Ranise, and Daniele Zucchelli, Decidability and undecidability results for Nelson-Oppen and rewritebased decision procedures, Proceedings of the Third International Joint Conference on Automated Reasoning (U. Furbach and N. Shankar, editors), Lecture Notes in Artificial Intelligence, vol. 4130, Springer-Verlag, 2006, pp. 513-527.

[11] Chen-Chung Chang and H. Jerome Keisler, Model theory, IIIrd ed., NorthHolland, Amsterdam-London, 1990.

[12] Eric Domenjoud, Francis Klay, and Christophe Ringeissen, Combination techniques for non-disjoint equational theories, Proceedings of the 12th International Conference on Automated Deduction (Nancy, France) (Alan Bundy, editor), Lecture Notes in Artificial Intelligence, vol. 814, Springer-Verlag, 1994, pp. 267-281.

[13] Camillo Fiorentini and Silvio Ghilardi, Combining word problems through rewriting in categories with products, Theoretical Computer Science, vol. 294 (2003), pp. 103-149.

[14] JEAN H. GALLIER, Logic for computer science: Foundations of automatic theorem proving, Harper \& Row, 1986.

[15] Silvio Ghilardi, Reasoners' cooperation and quantifier elimination, Technical Report 288-03, Dipartimento di Scienze dell'Informazione, Università degli Studi di Milano, 2003, Available on-line at http://homes.dsi.unimi.it/ ghilardi/allegati/eqfin.ps.

[16] - Model-theoretic methods in combined constraint satisfiability, Journal of Automated Reasoning, vol. 33 (2005), no. 3-4, pp. 221-249.

[17] Silvio Ghilardi and Marek Zawadowski, Sheaves, games and model completions, Trends in Logic, vol. 14, Kluwer Academic Publishers, 2002.

[18] Wilfrid Hodges, Model theory, Encyclopedia of Mathematics and its Applications, vol. 42, Cambridge University Press, Cambridge, 1993. 
[19] Marcus Kracht and Frank Wolter, Properties of independently axiomatizable bimodal logics, this JournaL, vol. 56 (1991), no. 4, pp. 1469-1485.

[20] Oliver Kutz, Carsten Lutz, Frank Wolter, and Michael Zakharyaschev, $\mathcal{E}$ connections of abstract description systems, Artificial Intelligence, vol. 156 (2004), pp. 1-73.

[21] Michael Makkai and Gonzalo E. Reyes, First-order categorical logic, Lecture Notes in Mathematics, vol. 611, Springer-Verlag, Berlin, 1977.

[22] Greg Nelson, Combining satisfiability procedures by equality-sharing, Automated theorem proving: After 25 years (W. W. Bledsoe and D. W. Loveland, editors), Contemporary Mathematics, vol. 29, American Mathematical Society, Providence, RI, 1984, pp. 201-211.

[23] Greg Nelson and Derek C. Oppen, Simplification by cooperating decision procedures, ACM Trans. on Programming Languages and Systems, vol. 1 (1979), no. 2, pp. 245-257.

[24] ToBias Nipkow, Combining matching algorithms: The regular case, Journal of Symbolic Computation, vol. 12 (1991), pp. 633-653.

[25] Derek C. Oppen, Complexity, convexity and combinations of theories, Theoretical Computer Science, vol. 12 (1980), pp. 291-302.

[26] Don Pigozzi, The join of equational theories, Colloquium Mathematicum, vol. 30 (1974), no. 1, pp. 15-25.

[27] MojZesz Presburger, Über die Vollständigkeit eines gewissen Systems der Arithmetik ganzer Zahlen, in welchem die Addition als einzige Operation hervortritt, Comptes rendus du congrès de mathématiciens des pays slaves (Warsaw, Poland), 1929.

[28] Manfred Schmidt-Schauss, Unification in a combination of arbitrary disjoint equational theories, Journal of Symbolic Computation, vol. 8 (1989), no. 1-2, pp. 51-100, Special issue on unification. Part II.

[29] Edith Spann, Complexity of modal logics, Ph.D. thesis, Department of Mathematics and Computer Science, University of Amsterdam, The Netherlands, 1993.

[30] Cesare Tinelli, Cooperation of background reasoners in theory reasoning by residue sharing, Journal of Automated Reasoning, vol. 30 (2003), no. 1, pp. 1-31.

[31] Cesare Tinelli and Christophe Ringeissen, Unions of non-disjoint theories and combinations of satisfiability procedures, Theoretical Computer Science, vol. 290 (2003), no. 1 , pp. $291-353$.

[32] Frank Wolter, Fusions of modal logics revisited, Advances in modal logic (M. Kracht, M. de Rijke, H. Wansing, and M. Zakharyaschev, editors), CSLI, Stanford, CA, 1998, pp. 361-379.

[33] Calogero Zarba, Combining multisets with integers, Procceedings of the 18th International Conference on Automated Deduction (CADE'18) (Andrei Voronkov, editor), Lecture Notes in Artificial Intelligence, vol. 2392, Springer-Verlag, 2002, pp. 363-376.

[34] Marek W. Zawadowski, Descent and duality, Ann. Pure Appl. Logic, vol. 71 (1995), no. 2 , pp. $131-188$.

INSTITUT FÜR THEORETISCHE INFORMATIK,

TU DRESDEN, GERMANY

E-mail: baader@tcs.inf.tu-dresden.de

DIPARTIMENTO DI SCIENZE DELL'INFORMAZIONE,

UNIVERSITÀ DEGLI STUDI DI MILANO, ITALY

E-mail: ghilardi@dsi.unimi.it 